\title{
Synthesis of (multi)silylalkynyl-substituted silsesquioxanes obtained via silylative coupling reaction
}

\author{
Monika Rzonsowska, ${ }^{\dagger, \ddagger}$ Kinga Zmudzińska, ${ }^{\dagger}$ Julia Duszczak, ${ }^{\dagger}$ Katarzyna Mituła, ${ }^{\dagger}$ \\ Beata Dudziec, ${ }^{*,+, \neq}$ Patrycja Żak ${ }^{\dagger}$
}

${ }^{\dagger}$ Department of Organometallic Chemistry, Faculty of Chemistry, Adam Mickiewicz University in Poznań, Uniwersytetu Poznańskiego 8, 61-614 Poznań, Poland

${ }^{\ddagger}$ Centre for Advanced Technologies, Adam Mickiewicz University in Poznań, Uniwersytetu Poznańskiego 10, 61-614 Poznań, Poland

\section{Contents:}

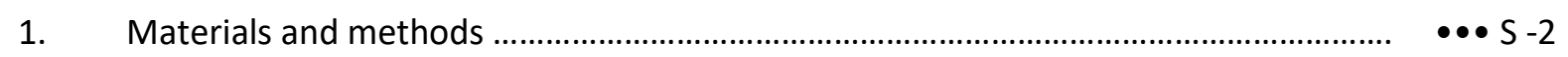

1.1 Chemicals .....................................................................................................

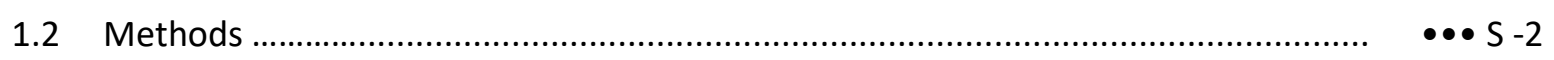

1.3 Screening Tests for silylative coupling of SQs with vinylsilanes $(\mathrm{S}-\mathrm{a}-\mathrm{S}-\mathrm{c}) \ldots \ldots \ldots \ldots \ldots \ldots \ldots \ldots \ldots$

2. Additional NMR analyses spectra for Screening Reactions.......................................... $\quad$ S -6

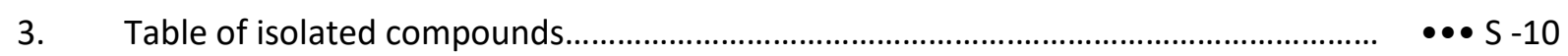

4. Data characterizing the obtained products and their ${ }^{1} \mathrm{H},{ }^{13} \mathrm{C},{ }^{29} \mathrm{Si}$ NMR spectra............ $\quad \cdots \mathrm{S}-12$ 


\section{Materials and methods}

\subsection{Chemicals}

The chemicals were purchased from the following sources: Hybrid Plastics for DDSQ tetrasilanol form $\left(\mathrm{C}_{48} \mathrm{H}_{44} \mathrm{O}_{14} \mathrm{Si}_{8}\right)$ (DDSQ-4OH) and trisilanols precursors of POSS ${ }^{\circledR}$; Sigma-Aldrich for dichloromethane (DCM), tetrahydrofuran (THF), diethyl ether, toluene, n-hexane, chloroform-d, hexamethylphosphoramide (HMPA), chlorosilanes, ethynyl magnesium bromide, calcium hydride, molecular sieves and anhydrous magnesium sulfate, triethylamine and silica gel 60 . Monoethynylsiloxysubstituted (SQ-1-SQ-4) and monoethynylsubstituted silsesquioxanes (SQ-5), diethynylsiloxysubstituted DDSQ (SQ-6) $)^{1}$ and tetraethynylsiloxysubstituted DDSQ (SQ-7) $)^{2}$ were prepared according to the literature procedures (see Scheme S-1). Chloroethynyldimethylsilane was prepared according to the literature procedure ${ }^{3}$. Ruthenium and rhodium complexes were prepared according to the literature procedure: $\left.\left[\mathrm{RuHCl}(\mathrm{CO})\left(\mathrm{PC} y_{3}\right)_{2}\right]^{4},\left[\mathrm{RuHCl}(\mathrm{CO})(\mathrm{SIDip})(\mathrm{PCy})_{3}\right)\right]^{5}, \mathrm{Rhl}\left(\mathrm{PPh}_{3}\right)_{3}{ }^{6}$.

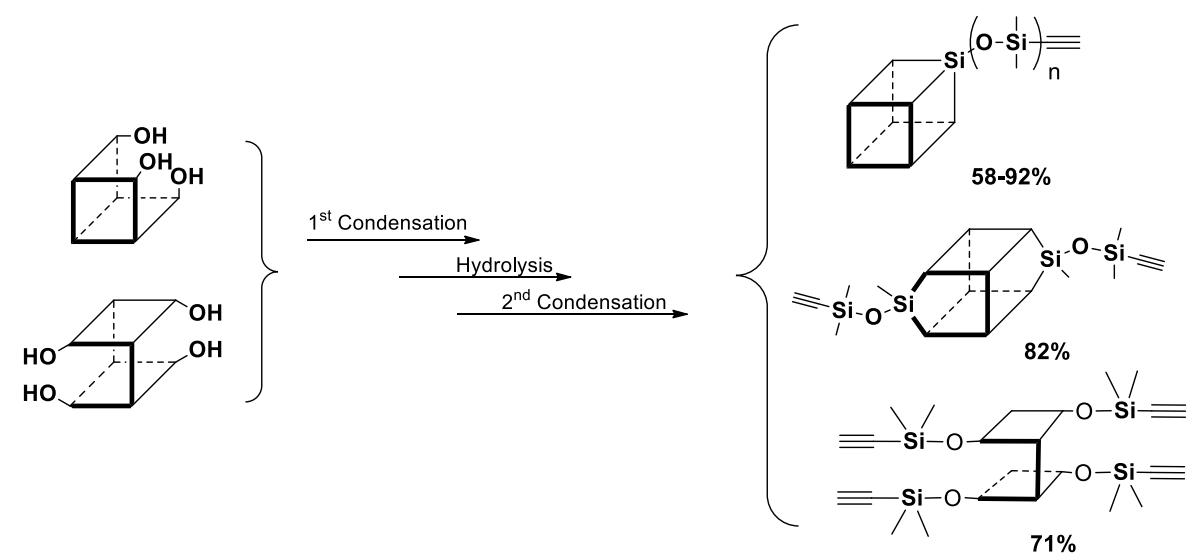

$1^{\text {st }}$ Condensation: for SQ-1-SQ-5 : $\mathrm{SiCl}_{4}, \mathrm{Et}_{3} \mathrm{~N}, \mathrm{THF}, \mathrm{RT}, 24 \mathrm{~h}$; for SQ-6: $\mathrm{HSiMeCl}_{2}, \mathrm{Et}_{3} \mathrm{~N}, \mathrm{THF}, \mathrm{RT}, 24 \mathrm{~h}$; for SQ-7: $\mathrm{HCCSiMe}{ }_{2} \mathrm{Cl}, \mathrm{Et}_{3} \mathrm{~N}, \mathrm{THF}, \mathrm{RT}, 24 \mathrm{~h}$; Hydrolysis only for SQ-1-SQ-4: THF/ $\mathrm{H}_{2} \mathrm{O}$ reflux, 24h; for SQ-6: $\mathrm{Pd}$ : $[\mathrm{Pd} / \mathrm{C}] 5 \mathrm{~mol} \%$ per $\mathrm{Si}-\mathrm{H}, \mathrm{THF}, 65^{\circ} \mathrm{C}, 48 \mathrm{~h}$ $2^{\text {nd }}$ Condensation only for SQ-1-SQ-4 and SQ-6: CISiMe ${ }_{2} \mathrm{CCH}, \mathrm{Et}_{3} \mathrm{~N}, \mathrm{THF}, \mathrm{RT}, 24 \mathrm{~h}$.

For SQ-5 ( $\mathrm{n}=0)$ : $\mathrm{HCCMgBr}, \mathrm{THF}, 45^{\circ} \mathrm{C}, 24 \mathrm{~h}$
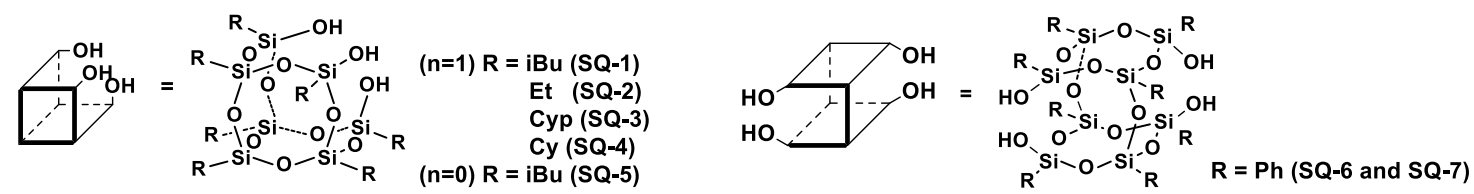

Scheme S 1. Synthetic procedure's routes for of the formation of mono-, di- and tetraethynylsiloxysilsesqioxanes (SQ-1 - SQ-7 $)^{1,2}$

\footnotetext{
${ }^{1}$ a) Dudziec, B.; Rzonsowska, M.; Marciniec, B.; Brząkalski, D. and Woźniak, B. Dalt. Trans. 2014, 43, 1320113207; b) Rzonsowska, M.; Dudziec, B. and Marciniec, B. Dalt. Trans. 2016, 45, 17187-17194; c) D. Brząkalski, M. Walczak, J. Duszczak, B. Dudziec, B. Marciniec Eur. J. Inorg. Chem. 2018, 45, 4905-4910

${ }^{2}$ Mituła, K.; Duszczak, J.; Brząkalski, D.; Dudziec, B. Chem. Commun. 2017, 53, 10370-10373

${ }^{3}$ Sukeda, M.; Ichikawa, S.; Matsuda, A.; Shuto, S. J. Org. Chem. 2003, 68 (9), 3465-3475

${ }^{4}$ Yi, C. S.; Lee, D. W.; Chen, Y. Organometallics 1999, 18 (11), 2043-2045

5 Żak, P.; Delaude, L.; Dudziec, B.; Marciniec, B. Chem. Commun. 2018, 54 (34), 4306-4309

${ }^{6}$ Jardine, F. H., Prog. Inorg. Chem., 1981, 28, 63
} 


\subsection{Methods}

All solvents were dried over sodium or $\mathrm{CaH}_{2}$ prior to use and stored under argon over type $4 \AA$ molecular sieves. All liquid substrates were also dried and degassed by bulb-to-bulb distillation.

All syntheses and manipulations were carried out under an argon atmosphere using standard Schlenk-line and vacuum techniques.

Elemental analyses were performed using a Vario EL III instrument.

Nuclear Magnetic Resonance (NMR) ${ }^{1} \mathrm{H},{ }^{13} \mathrm{C}$, and ${ }^{29} \mathrm{Si}$ Nuclear Magnetic Resonance (NMR) were performed on Brucker Ultra Shield 600, 400 and 300 spectrometers using $\mathrm{CDCl}_{3}$ as a solvent.

Chemical shifts are reported in ppm with reference to the residual solvent $\left(\mathrm{CHCl}_{3}\right)$ peaks for ${ }^{1} \mathrm{H}$ and

${ }^{13} \mathrm{C}$ and to TMS for ${ }^{29} \mathrm{Si}$. 


\subsection{Screening Tests for silylative coupling of SQs with vinylsilanes (S-a - S-c)}

The model experiments using trimethylvinylsilanes with 1-ethynyldimethylsiloxy(heptaisobutyl)octasilsesquioxane (SQ-1, for monosubstituted products) and 9,19bis[ethynyldimethylsiloxymethyl]octa(phenyl)deca-silsesquioxane (SQ-6, for disubstituted products) aimed at optimization of reaction conditions were performed.

\section{Screening Reaction}

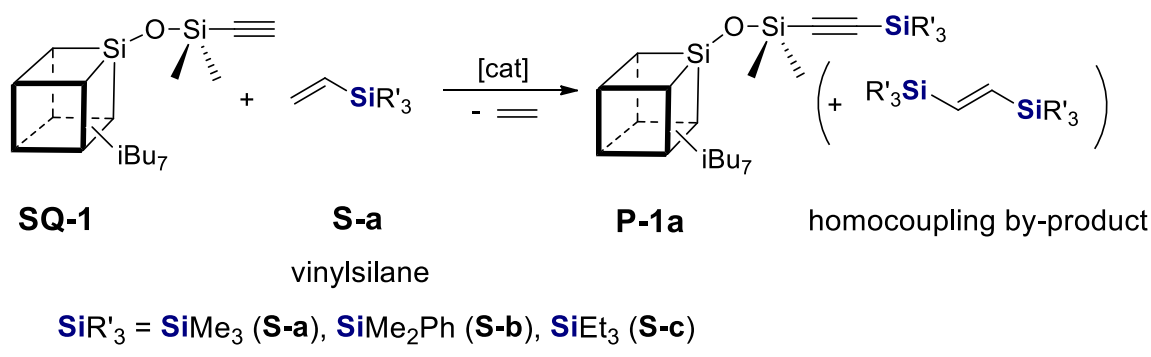

Table S 1. Screening Tests for silylative coupling of SQ-1 with vinylsilanes (S-a - S-c)

\begin{tabular}{|c|c|c|c|}
\hline Entry & Catalyst & $\begin{array}{l}\text { [cat]:[SQ-1] } \\
:[\text { vinylsilane] }\end{array}$ & 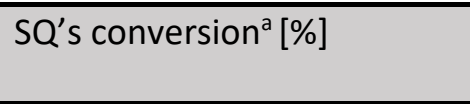 \\
\hline 1 & $\operatorname{Rhl}\left(\mathrm{PPh}_{3}\right)_{3}(\mathrm{II})$ & $5 \times 10^{-2}: 1: 3(\mathrm{~S}-\mathrm{a})$ & $\begin{array}{l}\text { dimerization product of SQ-1 } \\
\text { (see Figure S-2 - S-4) }\end{array}$ \\
\hline 2 & & $10^{-2}: 1: 2(\mathrm{~S}-\mathrm{a})$ & 65 \\
\hline 4 & $\begin{array}{l}\mathrm{PCy}_{3} \\
=\left[\mathrm{RuHCl}(\mathrm{CO})(\mathrm{NHC})\left(\mathrm{PCY}_{3}\right)\right] \\
\text { (NHC=SIDip: } 1,3-\text { bis }(2,6- \\
\text { diisopropylphenyl)imidazole) }\end{array}$ & $5 \times 10^{-2}: 1: 3$ (S-a) & 99 \\
\hline 5 & {$\left[\mathrm{RuHCl}(\mathrm{CO})\left(\mathrm{PCy}_{3}\right)_{2}\right](\mathrm{I})$} & $10^{-2}: 1: 2(S-a)$ & 80 \\
\hline 6 & & $5 \times 10^{-2}: 1: 3(\mathrm{~S}-\mathrm{a})$ & $>99$ \\
\hline 7 & & $5 \times 10^{-2}: 1: 4$ (S-a) & $>99^{b}$ \\
\hline 8 & & $5 \times 10^{-2}: 1: 3$ (S-b) & $>99^{b}$ \\
\hline 9 & & $5 \times 10^{-2}: 1: 3(\mathrm{~S}-\mathrm{c})$ & $>99$ \\
\hline \multicolumn{4}{|c|}{$\begin{array}{l}\text { Reaction conditions: toluene, } \mathrm{T}=120^{\circ} \mathrm{C}, 24 \mathrm{~h} \text {. } \\
{ }^{\mathrm{a}} \mathrm{S} \mathrm{Q}^{\prime} \mathrm{s} \text { conversion determined on the basis of }{ }^{1} \mathrm{H} \text { NMR } \\
{ }^{\mathrm{b}} \text { Accompanied by traces of vinylsilane homocoupling } b\end{array}$} \\
\hline
\end{tabular}




\section{Screening Reaction}

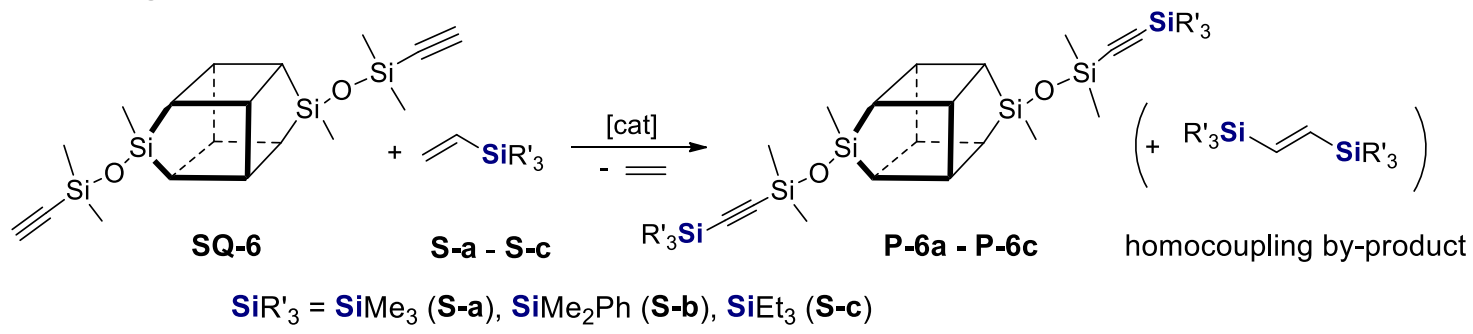

Table S 2. Screening Tests for silylative coupling of SQ-6 with vinylsilanes (S-a - S-c)

\begin{tabular}{|c|c|c|c|c|}
\hline Entry & Catalyst & $\begin{array}{l}\text { [cat]:[SQ-6] } \\
:[\text { vinylsilane] }\end{array}$ & Time [h] & SQ's conversion $^{\mathrm{a}}[\%]$ \\
\hline $\begin{array}{l}1 \\
2 \\
3 \\
4 \\
5 \\
6\end{array}$ & {$\left[\mathrm{RuHCl}(\mathrm{CO})\left(\mathrm{PCy}_{3}\right)_{2}\right](\mathrm{I})$} & $\begin{array}{l}10^{-2}: 1: 3 \text { (S-a) } \\
5 \times 10^{-2}: 1: 3 \text { (S-a) } \\
5 \times 10^{-2}: 1: 5 \text { (S-a) } \\
5 \times 10^{-2}: 1: 5 \text { (S-a) } \\
5 \times 10^{-2}: 1: 5 \text { (S-b) } \\
5 \times 10^{-2}: 1: 10(\text { S-a) }\end{array}$ & $\begin{array}{l}24 \mathrm{~h} \\
24 \\
24 \\
48 \\
48 \\
48\end{array}$ & $\begin{array}{l}30 \\
60 \\
85 \\
99 \\
99^{b} \\
>99^{b}\end{array}$ \\
\hline 7 & \multirow{5}{*}{$=\left[\mathrm{RuHCl}(\mathrm{CO})(\mathrm{NHC})\left(\mathrm{PCy}_{3}\right)\right]_{\text {(III) }}$} & $10^{-2}: 1: 3$ (S-a) & 24 & 33 \\
\hline 8 & & $5 \times 10^{-2}: 1: 5$ (S-a) & 24 & 85 \\
\hline 9 & & $5 \times 10^{-2}: 1: 5$ (S-a) & 48 & 99 \\
\hline 10 & & $5 \times 10^{-2}: 1: 5$ (S-b) & 48 & $>99$ \\
\hline 11 & & $5 \times 10^{-2}: 1: 5$ (S-c) & 48 & $>99^{b}$ \\
\hline \multicolumn{5}{|c|}{$\begin{array}{l}\text { Reaction conditions: toluene, } \mathrm{T}=120^{\circ} \mathrm{C} \\
\text { a } \mathrm{SQ}^{\prime} \mathrm{s} \text { conversion determined on the basis of }{ }^{1} \mathrm{H} N \mathrm{NMR}\end{array}$} \\
\hline
\end{tabular}


2 Additional NMR analyses spectra for Screening Reactions

NMR spectra of post-reaction mixtures of SQ-2 and S-a to evaluate the absence/presence of SQ-2/P-1a

$13 \mathrm{C}$ NMR $(75 \mathrm{MHz}, \mathrm{CDCl} 3)$
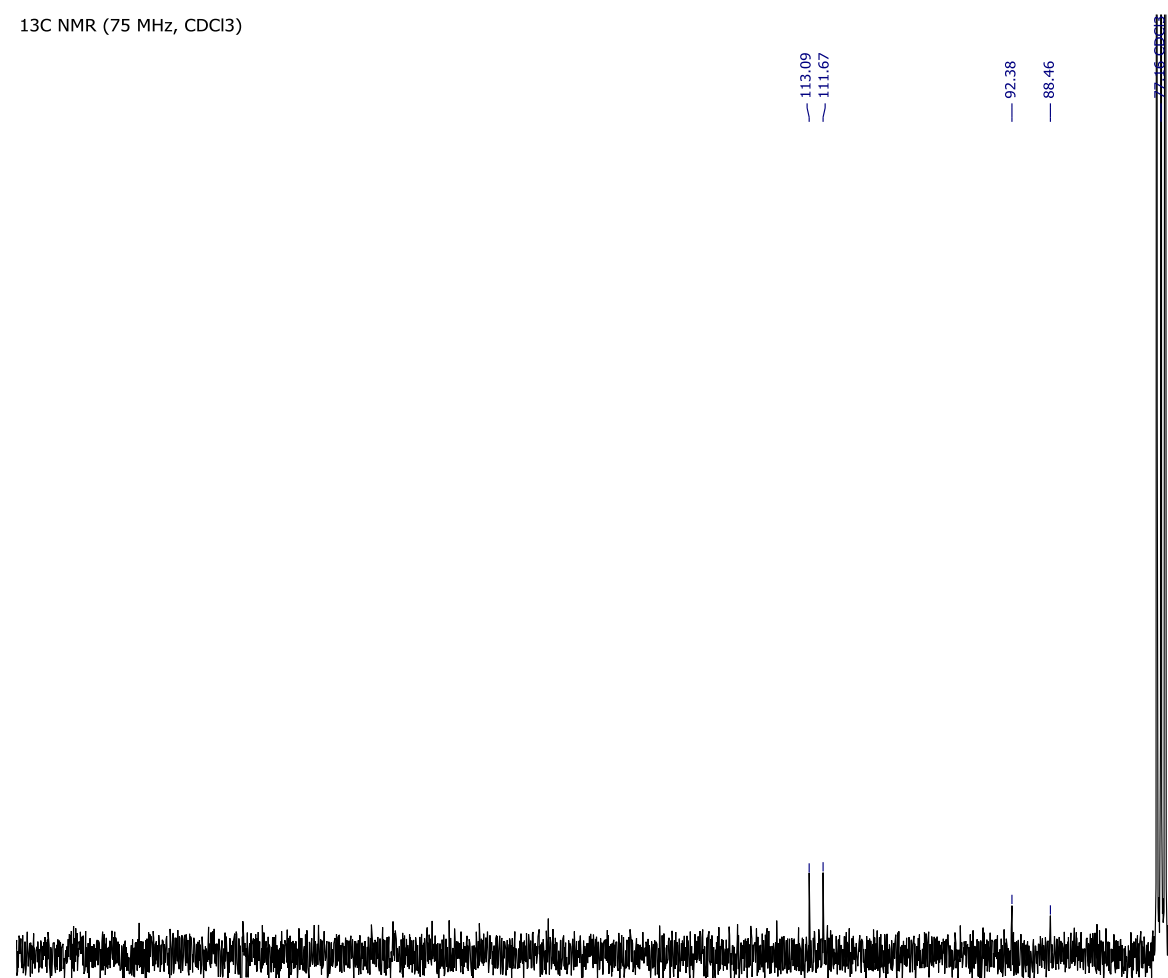

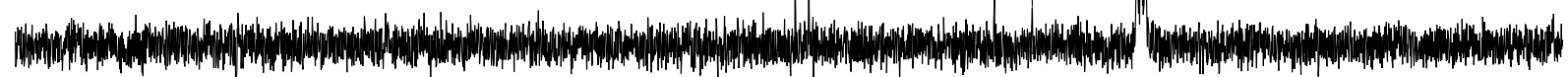

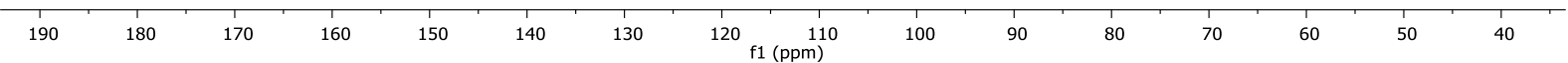

Figure S-1. Selected region of ${ }^{13} \mathrm{C}$ NMR spectrum for post reaction mixture of SQ-2 and S-a in the presence of $\left[\mathrm{RuHCl}(\mathrm{CO})(\mathrm{SIDip})\left(\mathrm{PCy}_{3}\right)\right](\mathrm{III})\left(10^{-2} \mathrm{~mol}\right)$ 
NMR analysis of reaction of SQ-1 and S-a in the presence of $\mathrm{RhI}\left(\mathrm{PPh}_{3}\right)_{3}$ (II), resulting in the formation of (E)-1,4-disilsesquioxylsubstitiuted but-1-en-3-yne (dimerization product)

(E)-1,4-bis $\{1$-ethynyldimethylsiloxy-3,5,7,9,11,13,15-hepta(isobutyl)pentacyclo$\left[9.5 \cdot 1 \cdot 13,9.1^{5,15} \cdot 1^{7,13}\right]$ octasiloxyl)-but-1-en-3-yne

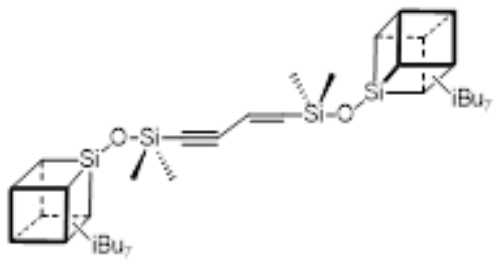

$1 \mathrm{H}$ NMR $(300 \mathrm{MHz}, \mathrm{CDCl} 3)$
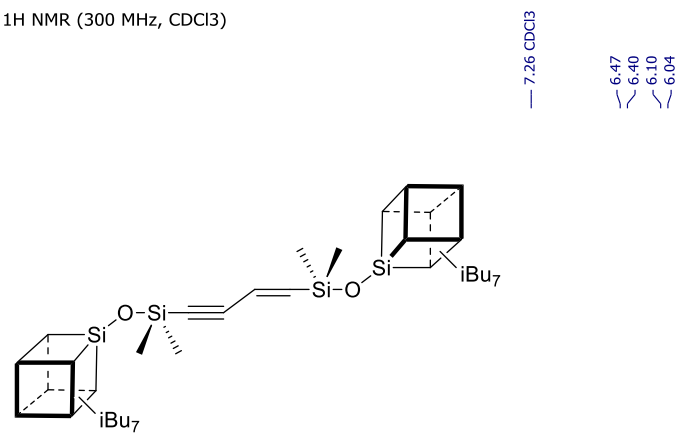

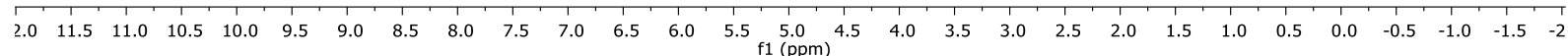

Figure S-2. ${ }^{1} \mathrm{H}$ NMR spectrum for product after reaction of SQ-1 and S-a in the presence of $\mathrm{Rhl}\left(\mathrm{PPh}_{3}\right)_{3}$ (II), resulting in the formation of (E)-1,4-disilsesquioxylsubstitiuted but-1-en-3-yne (dimerization product $)^{7}$

7 Żak, P.; Bołt, M.; Dudziec, B.; Kubicki, M. Dalt. Trans. 2019, 48 (8), 2657-2663. 

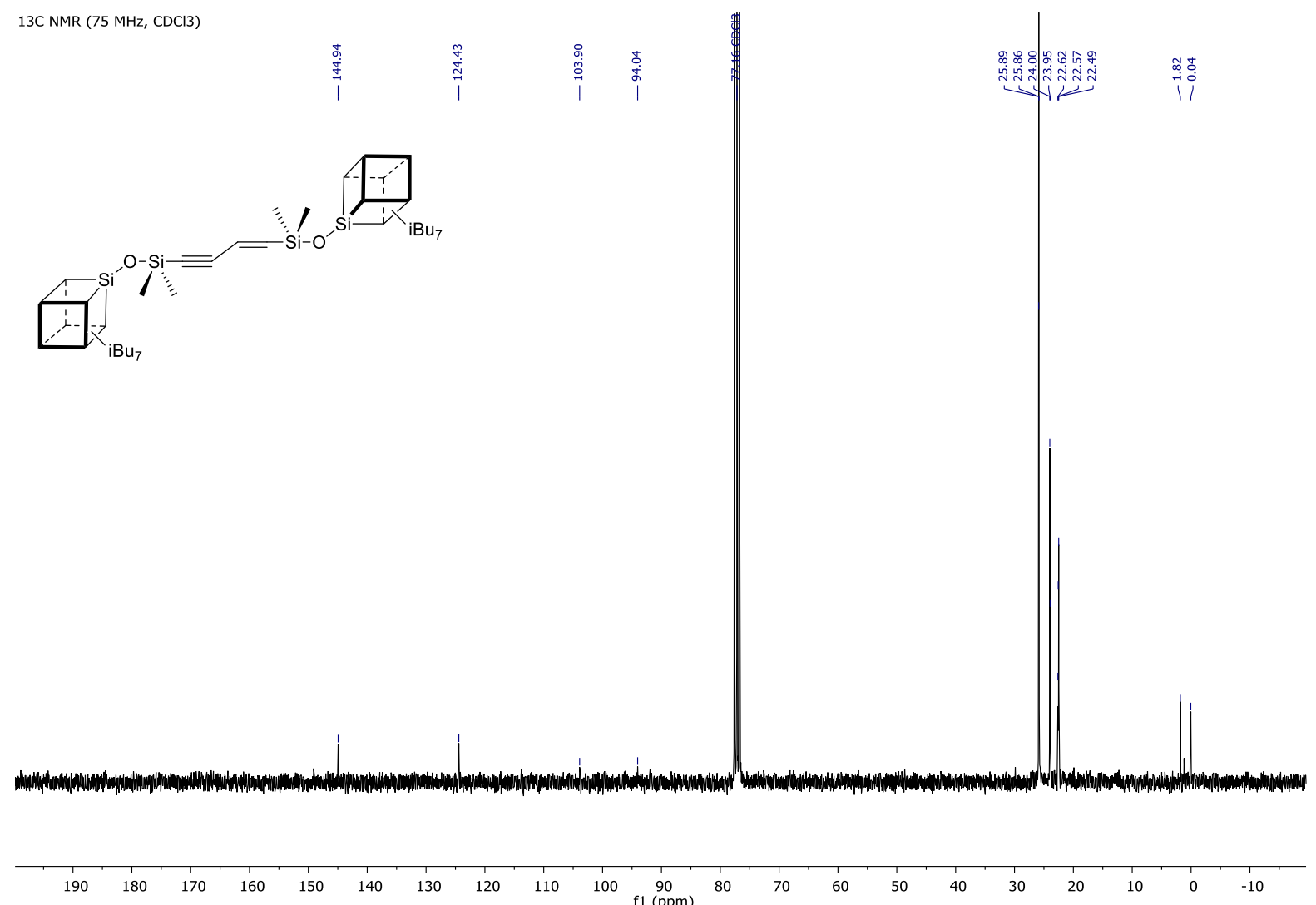

Figure S-3. ${ }^{13} \mathrm{C}$ NMR spectrum for product after reaction of SQ-1 and $\mathbf{S}$-a in the presence of $\mathrm{RhI}\left(\mathrm{PPh}_{3}\right)_{3}$ (II), resulting in the formation of (E)-1,4-disilsesquioxylsubstitiuted but-1-en-3-yne (dimerization product $)^{4}$ 


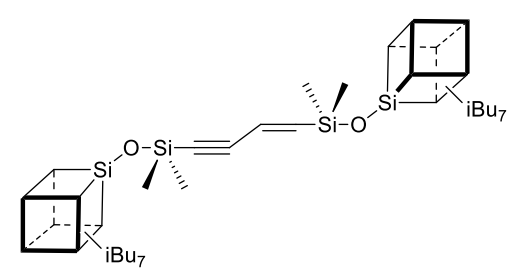

\begin{tabular}{llllllllllllllllllllllllllllll}
\hline 00 & 90 & 80 & 70 & 60 & 50 & 40 & 30 & 20 & 10 & 0 & -10 & -20 & -30 & -40 & -50 & -60 & -70 & -80 & -90 & -100 & -110 & -120 & -130 & -140 & -1
\end{tabular}

Figure S-4. ${ }^{29} \mathrm{Si}$ NMR spectrum for product after reaction of SQ-1 and S-a in the presence of $\mathrm{Rhl}\left(\mathrm{PPh}_{3}\right)_{3}$ (II), resulting in the formation of $(E)-1,4$-disilsesquioxylsubstitiuted but-1-en-3-yne (dimerization product) ${ }^{4}$

$1 \mathrm{H}$ NMR (300 MHz, CDCl3)
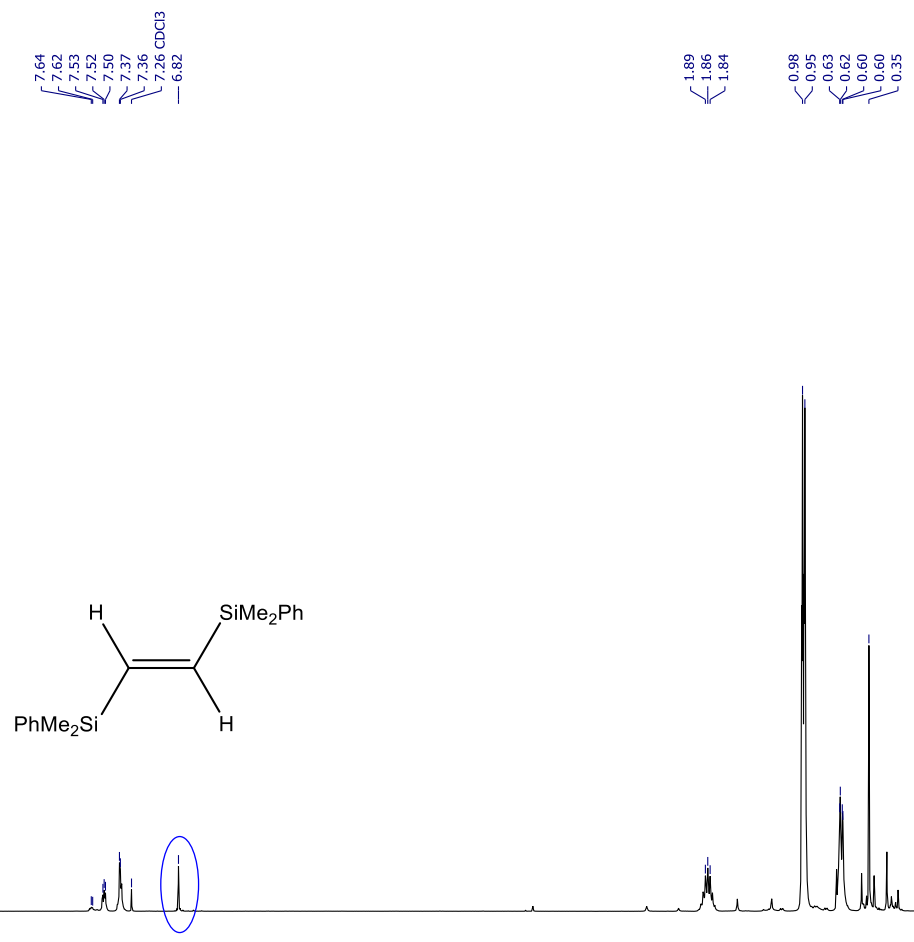

$\begin{array}{lllllllllllllllllllllllllllllllll}11.5 & 11.0 & 10.5 & 10.0 & 9.5 & 9.0 & 8.5 & 8.0 & 7.5 & 7.0 & 6.5 & 6.0 & 5.5 & 5.0 & 4.5 & 4.0 & 3.5 & 3.0 & 2.5 & 2.0 & 1.5 & 1.0 & 0.5 & 0.0 & -0.5 & -1.0 & -1.5 & -2\end{array}$

Figure S-5. ${ }^{1} \mathrm{H}$ NMR spectrum for the post reaction mixture for SQ-1 and $\mathbf{S}-\mathbf{b}$ ([SQ-1]:[S-b]=1:3) in the presence of (I) $\left(5 \times 10^{-2} \mathrm{~mol}\right)$ 
3. Table of obtained products

Table S 3. Silylative coupling of SQs' with vinylsilanes (S-a $-\mathrm{S}-\mathrm{d})$ with respective products

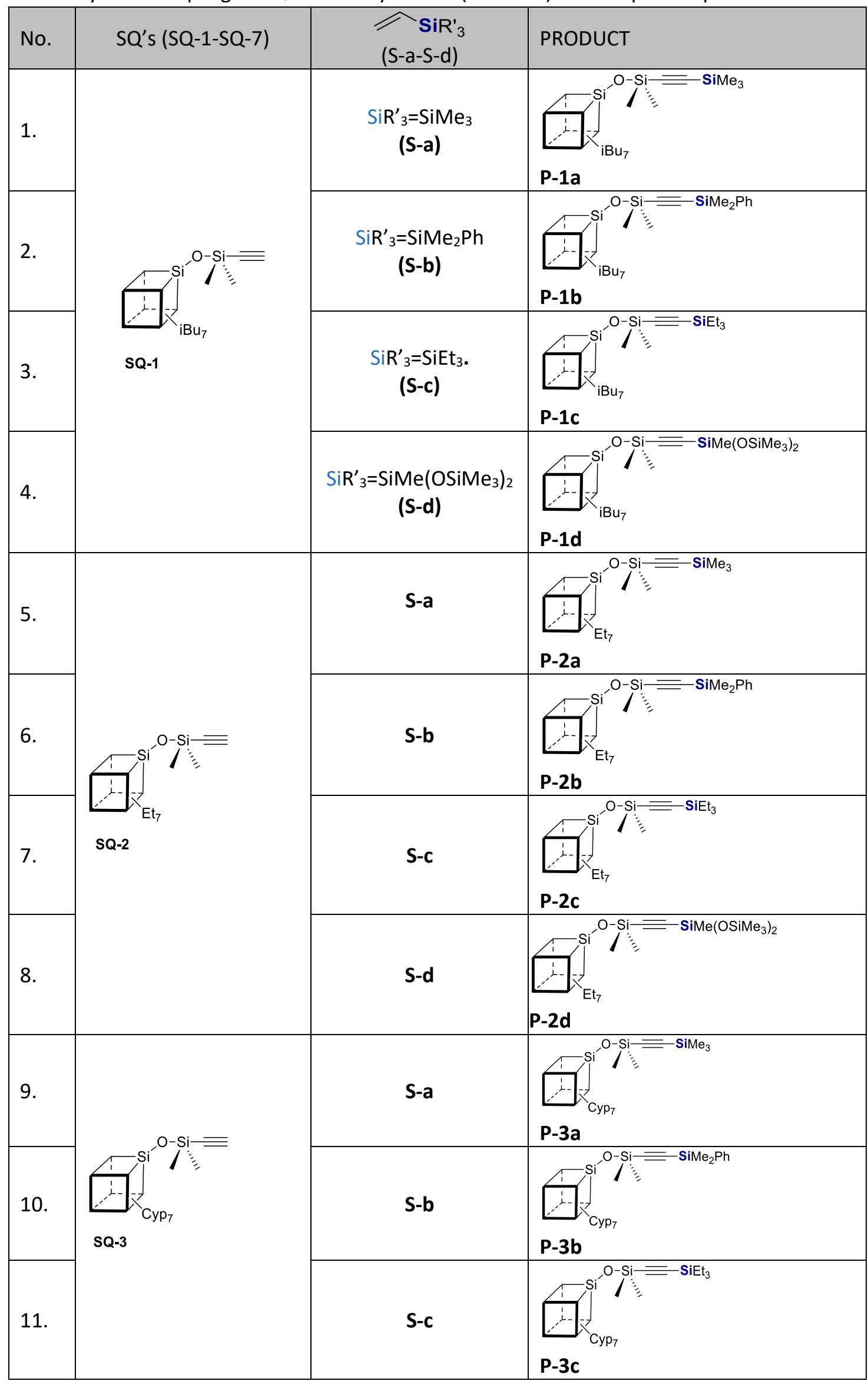




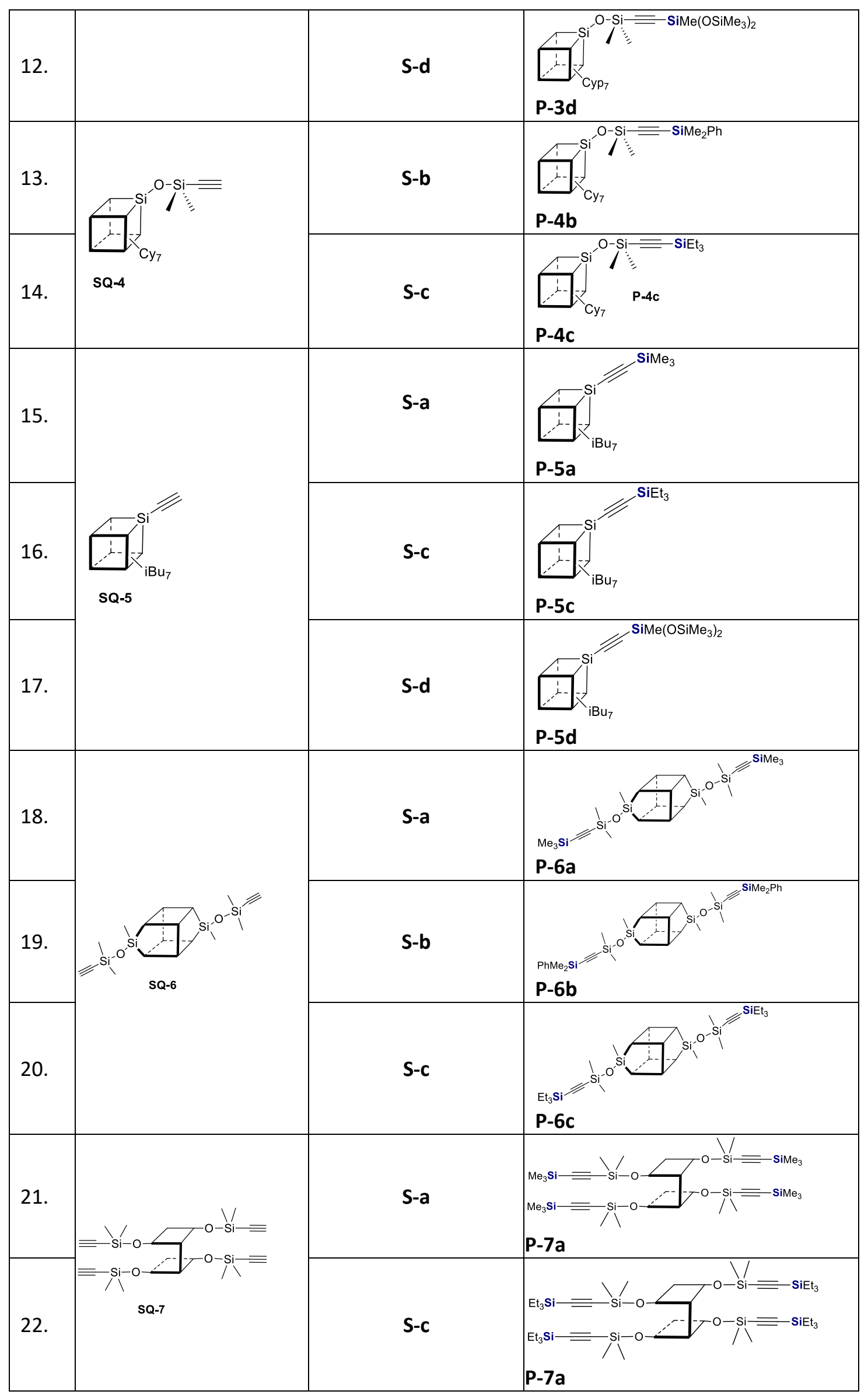




\section{Analyses of obtained products}

The Isolated yields and NMR spectra are presented for compounds obtained in the presence of $\left[\mathrm{RuHCl}(\mathrm{CO})\left(\mathrm{PCy}_{3}\right)_{2}\right](\mathrm{I})$.

\section{P-1a}

White solid. Isolated yield $77 \%$.

${ }^{1} \mathrm{H}$ NMR $\left(\mathrm{CDCl}_{3}, 500 \mathrm{MHz}\right): \delta$ 1.92-1.84 (m, 7H, -CH- (iBu)); 0.98-0.96 (m, 42H, $\left.-\mathrm{CH}_{3}(\mathrm{iBu})\right), 0.64-0.59$ $\left(\mathrm{m}, 14 \mathrm{H},-\mathrm{CH}_{2}-(\mathrm{iBu})\right), 0.27\left(\mathrm{~s}, 6 \mathrm{H},-\mathrm{SiMe}_{2}\right), 0.18\left(\mathrm{~s}, 9 \mathrm{H},-\mathrm{SiMe}_{3}\right)$.

${ }^{13} \mathrm{C}$ NMR $\left(\mathrm{CDCl}_{3}, 125 \mathrm{MHz}\right): \delta 112.96(\mathrm{C} \equiv \mathrm{C}), 111.79(\mathrm{C} \equiv \mathrm{C}), 25.89,25.86,24.01,23.95,22.62,22.52$, $1.90,-0.01$.

${ }^{29}$ Si NMR $\left(\mathrm{CDCl}_{3}, 99 \mathrm{MHz}\right): \delta-17.57,-18.66,-66.95,-67.88,-109.89$.

EA: Anal. calcd for $\mathrm{C}_{35} \mathrm{H}_{78} \mathrm{O}_{13} \mathrm{Si}_{10}(\%): \mathrm{C}, 42.55, \mathrm{H}, 7.96$; found: $\mathrm{C}, 42.69 ; \mathrm{H}, 8.03$.
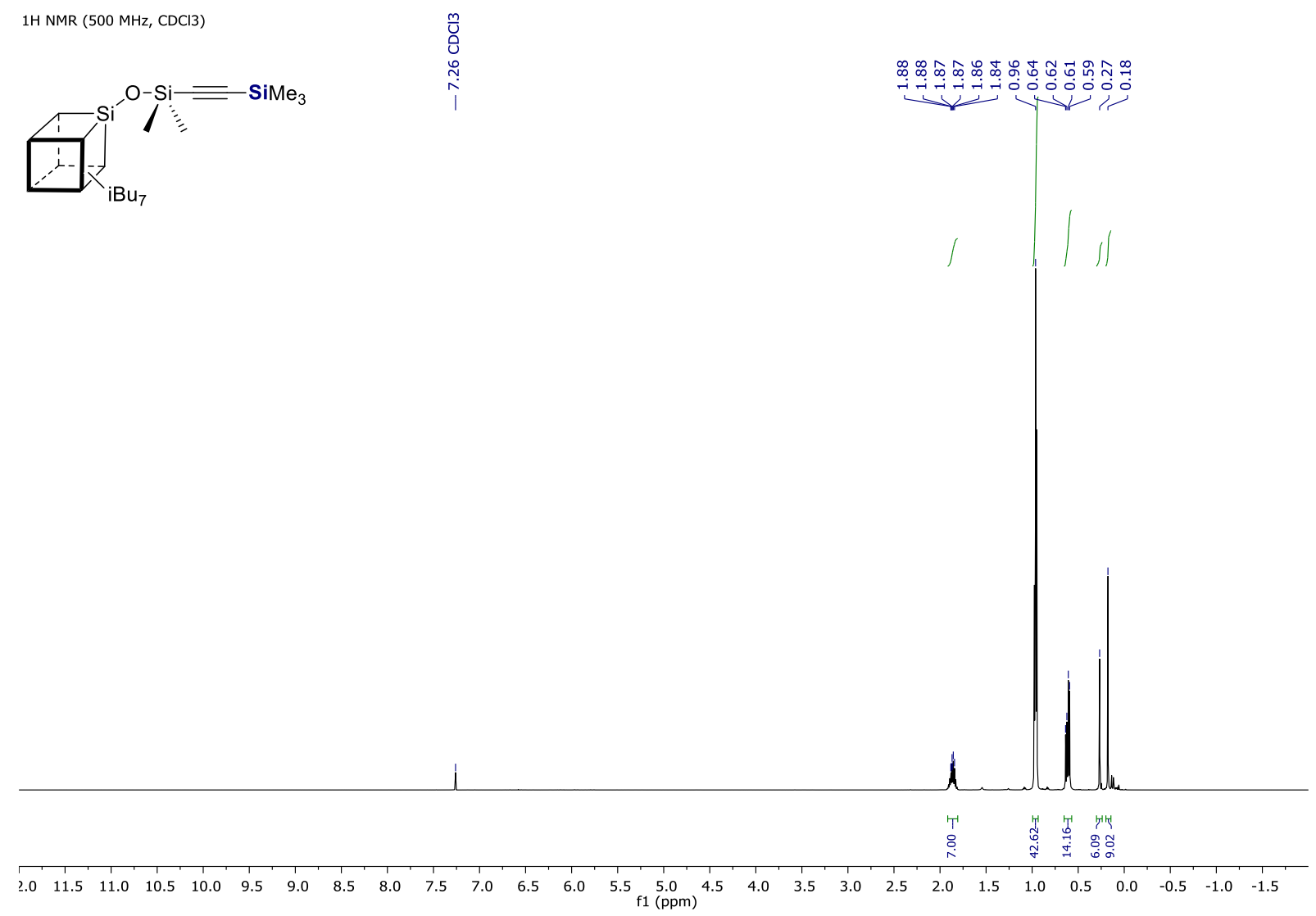

Figure S-6. ${ }^{1} \mathrm{H}$ NMR spectrum $\left(500 \mathrm{MHz}, \mathrm{CDCl}_{3}\right)$ of product P-1a. 


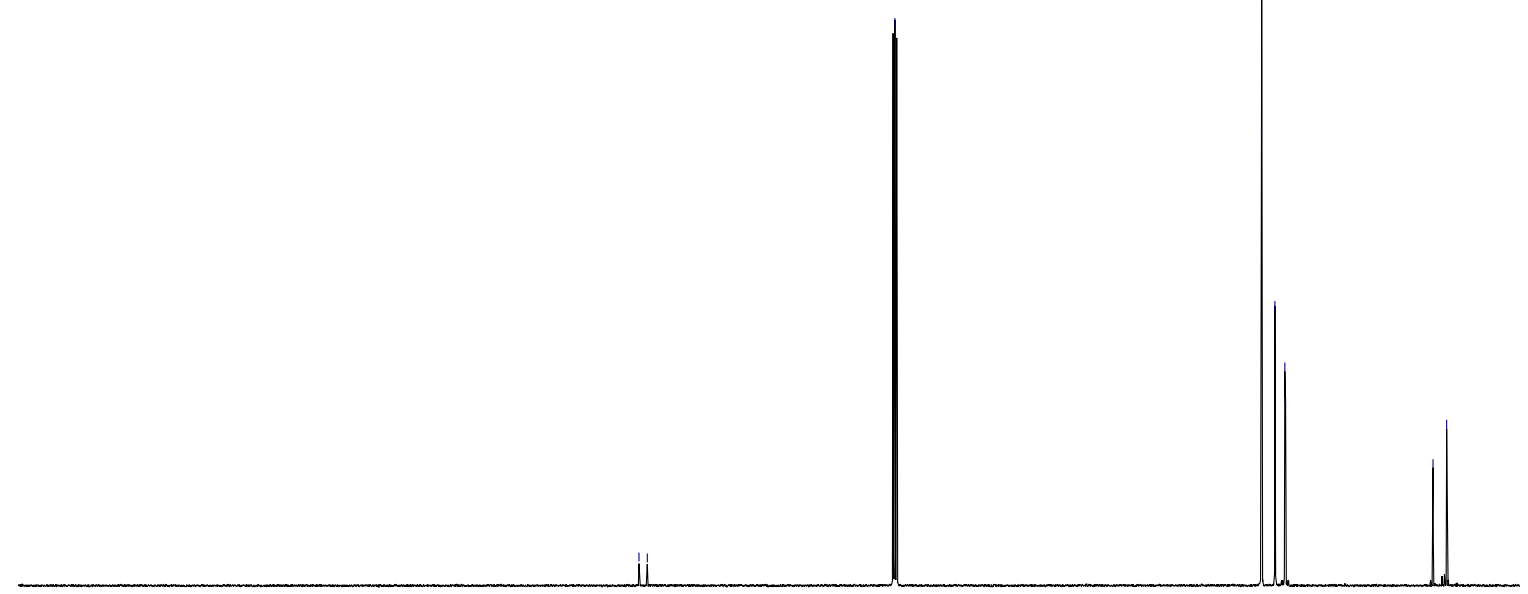

$\begin{array}{llllllllll}190 & 180 & 170 & 160 & 150 & 140 & 130 & 120 & 110 & \begin{array}{c}100 \\ \text { f1 (ppm) }\end{array}\end{array}$

Figure S-7. ${ }^{13} \mathrm{C}$ NMR spectrum $\left(125 \mathrm{MHz}, \mathrm{CDCl}_{3}\right)$ of product $\mathrm{P}-1 \mathrm{a}$.
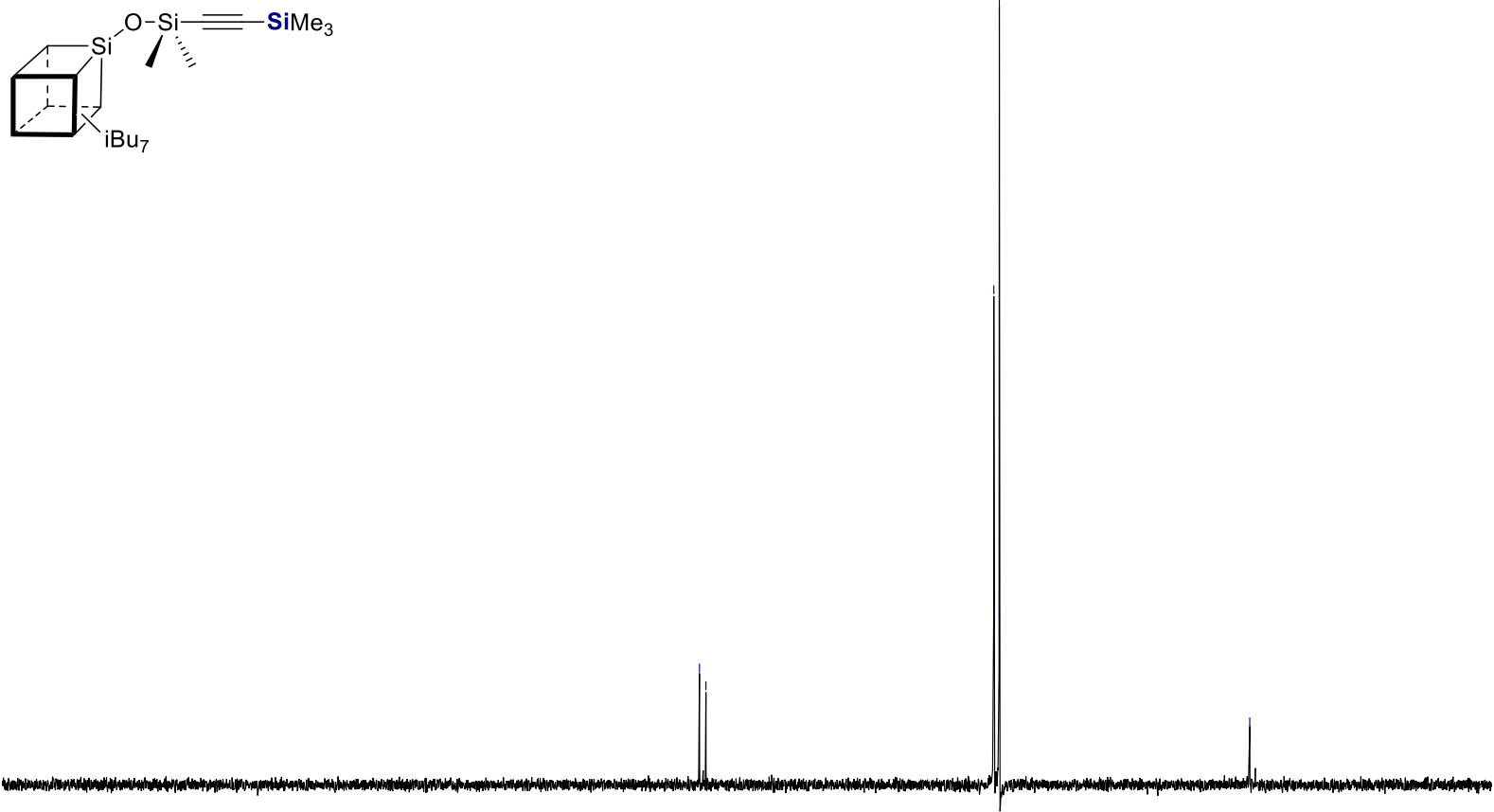

Figure S-8. ${ }^{29} \mathrm{Si} \mathrm{NMR} \mathrm{spectrum}\left(99 \mathrm{MHz}, \mathrm{CDCl}_{3}\right)$ of product $\mathrm{P}-1 \mathrm{a}$. 


\section{P-1b}

White solid. Isolated Yield $76 \%$. Product accompanied by homocoupling product of S-b, i.e. 1,2-bis(dimethylphenylsilyl)ethene (HP)

${ }^{1} \mathbf{H}$ NMR $\left(\mathrm{CDCl}_{3}, 500 \mathrm{MHz}\right): \delta$ 7.66-7.50 (m, 2H, -Ph), 7.39-7.35 (m, 3H, -Ph), 1.92-1.82 (m, 7H, -CH(iBu)), 0.98-0.94 (m, 42H, - $\left.\mathrm{CH}_{3}(\mathrm{iBu})\right), 0.63-0.60(\mathrm{~m}, 14 \mathrm{H},-\mathrm{CH}-(\mathrm{iBu})), 0.42\left(\mathrm{~s}, 6 \mathrm{H},-\mathrm{SiMe}_{2} \mathrm{Ph}\right), 0.36(\mathrm{~s}$, $12 \mathrm{H}$ - homocoupling product - HP), 0.31 (s, $\left.6 \mathrm{H},-\mathrm{SiMe}_{2}\right)$.

${ }^{13} \mathrm{C}$ NMR $\left(\mathrm{CDCl}_{3}, 125 \mathrm{MHz}\right): \delta 136.75,133.87,129.54,128.00,113.92(\mathrm{C} \equiv \mathrm{C}), 110.55(\mathrm{C} \equiv \mathrm{C}), 25.86$, 24.01, 23.95, 22.62, 1.89, -0.71, -2.92 (HP).

${ }^{29} \mathrm{Si}$ NMR $\left(\mathrm{CDCl}_{3}, 79 \mathrm{MHz}\right): \delta-7.93,-11.75$ (HP), -17.41, -66.71, -66.92, -67.82, -100.99.

EA: Anal. calcd for $\mathrm{C}_{40} \mathrm{H}_{80} \mathrm{O}_{13} \mathrm{Si}_{10}(\%): \mathrm{C}, 45.76, \mathrm{H}, 7.68$; found: $\mathrm{C}, 46.09 ; \mathrm{H}, 7.93$.

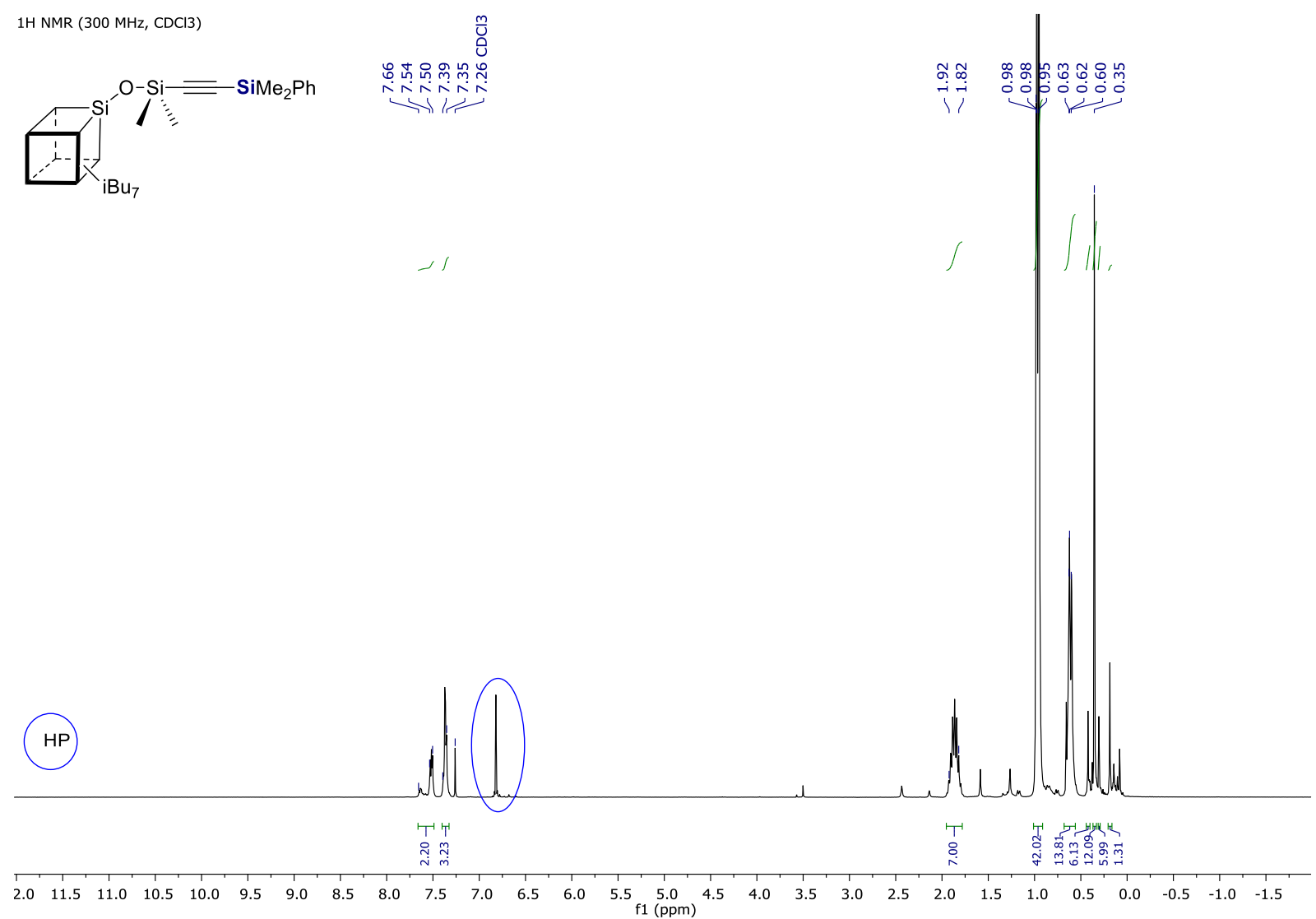

Figure S-9. ${ }^{1} \mathrm{H}$ NMR spectrum $\left(300 \mathrm{MHz}, \mathrm{CDCl}_{3}\right)$ of product $\mathrm{P}-1 \mathrm{~b}$. 

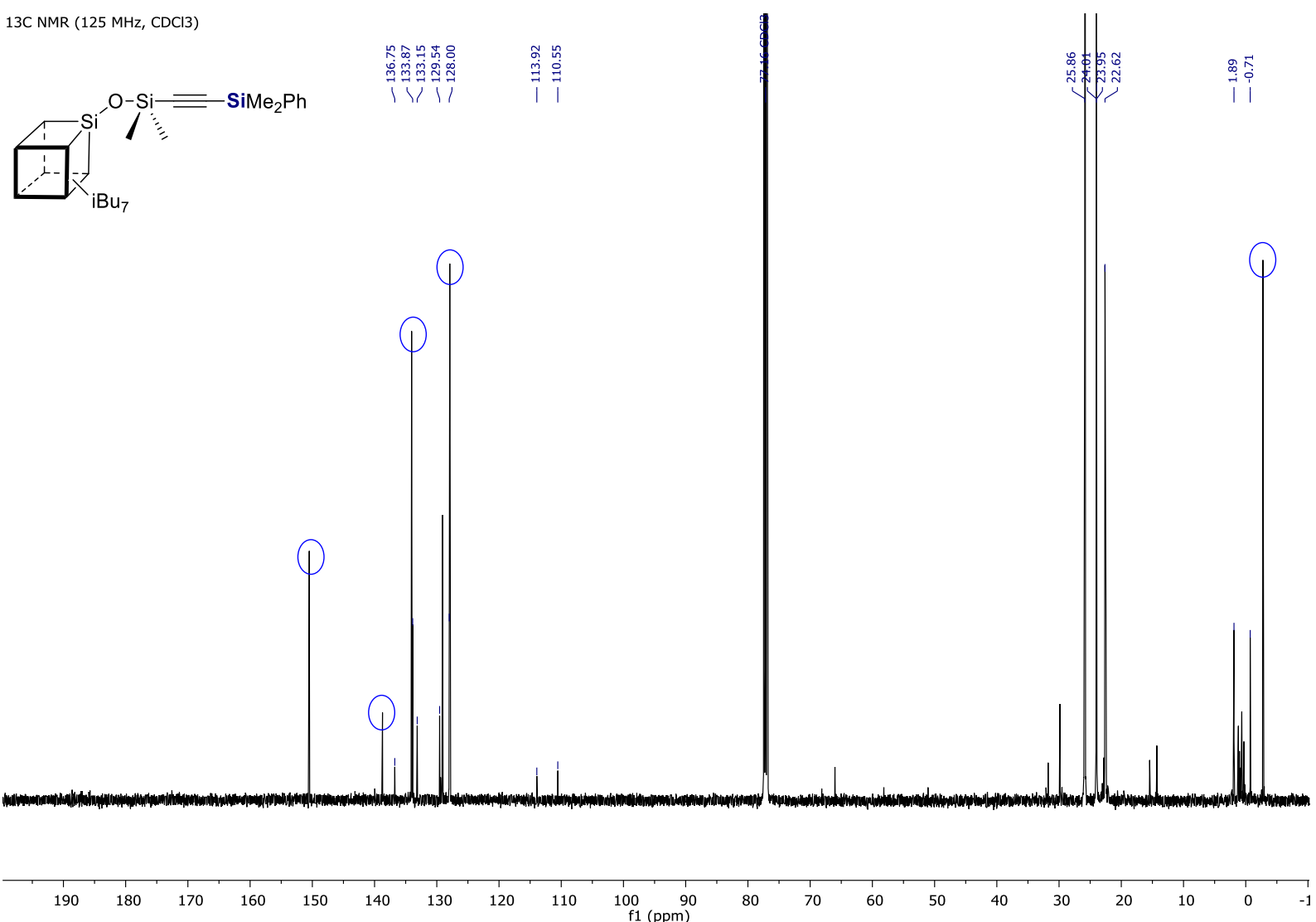

Figure S-10. ${ }^{13} \mathrm{C}$ NMR spectrum (125 MHz, $\left.\mathrm{CDCl}_{3}\right)$ of product $\mathrm{P}-1 \mathrm{~b}$.

29Si NMR (79 MHz, CDCl3)
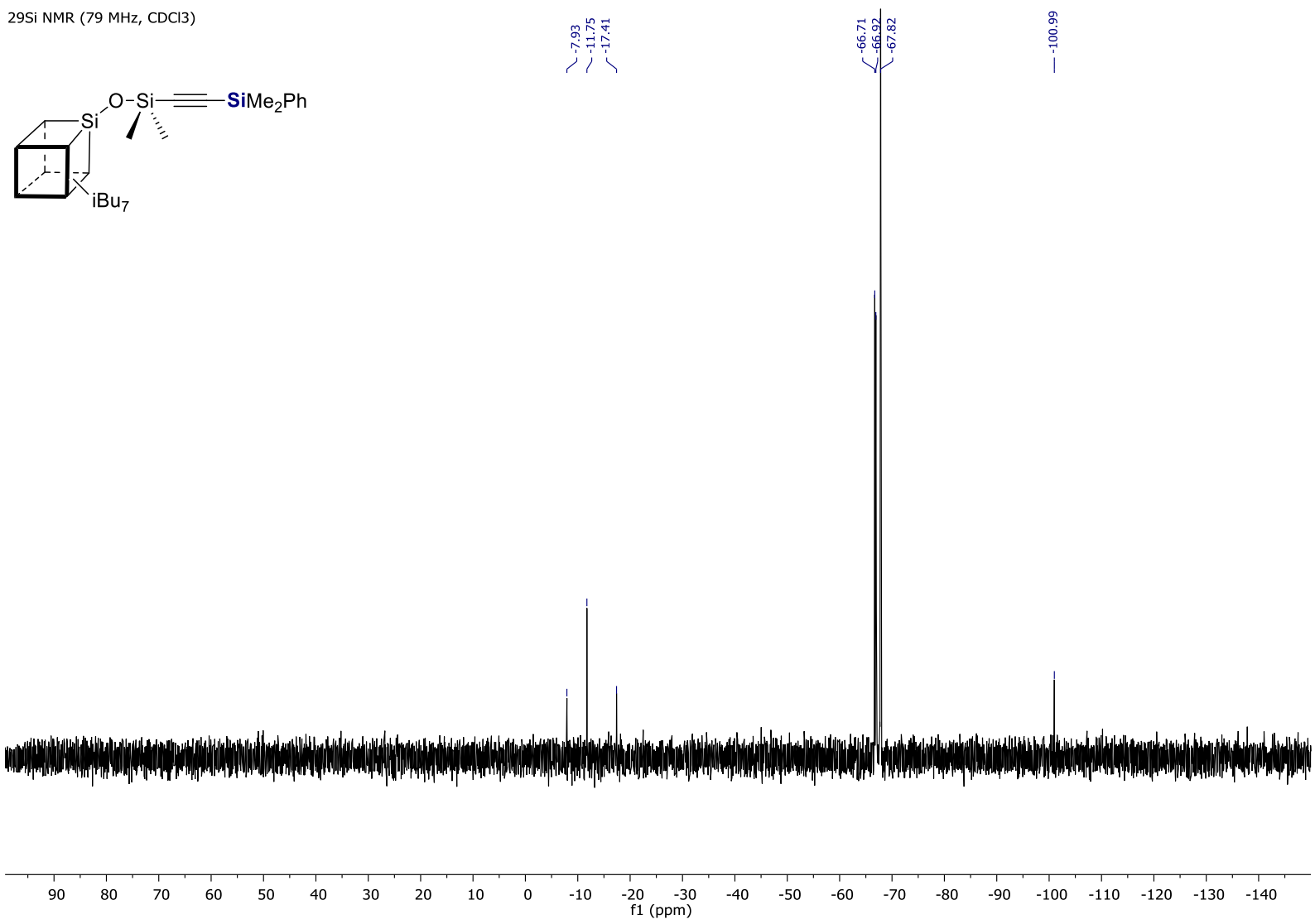

Figure S-11. ${ }^{29} \mathrm{Si} \mathrm{NMR} \mathrm{spectrum}\left(79 \mathrm{MHz}, \mathrm{CDCl}_{3}\right)$ of product P-1b. 
White solid. Isolated yield $72 \%$.

${ }^{1} \mathrm{H}$ NMR $\left(\mathrm{CDCl}_{3}, 300 \mathrm{MHz}\right): \delta 1.88-1.83(\mathrm{~m}, 7 \mathrm{H},-\mathrm{CH}-(\mathrm{iBu})), 0.94-0.97\left(\mathrm{~m}, 51 \mathrm{H},-\mathrm{CH}_{3}(\mathrm{iBu})+-\mathrm{CH}_{3}(\mathrm{Et})\right)$, 0.61-0.59 (m, 20H, $\left.-\mathrm{CH}_{2}-(\mathrm{iBu})+-\mathrm{CH}_{2}-(\mathrm{Et})\right), 0.27\left(\mathrm{~s}, 6 \mathrm{H},-\mathrm{SiMe}_{2}\right)$.

${ }^{13} \mathrm{C}$ NMR $\left(\mathrm{CDCl}_{3}, 100 \mathrm{MHz}\right): \delta 113.37(\mathrm{C} \equiv \mathrm{C}), 110.51(\mathrm{C} \equiv \mathrm{C}), 25.86,24.03,24.02,22.96,22.62,7.57$, 4.38, 1.98 .

${ }^{29} \mathrm{Si} \mathrm{NMR}\left(\mathrm{CDCl}_{3}, 79 \mathrm{MHz}\right): \delta-7.92,-17.85,-66.97,-67.90,-110.01$.

EA: Anal. calcd for $\mathrm{C}_{38} \mathrm{H}_{84} \mathrm{O}_{13} \mathrm{Si}_{10}$ (\%):C, 44.31, $\mathrm{H}, 8.22$; found: $\mathrm{C}, 44.39 ; \mathrm{H}, 8.37$.
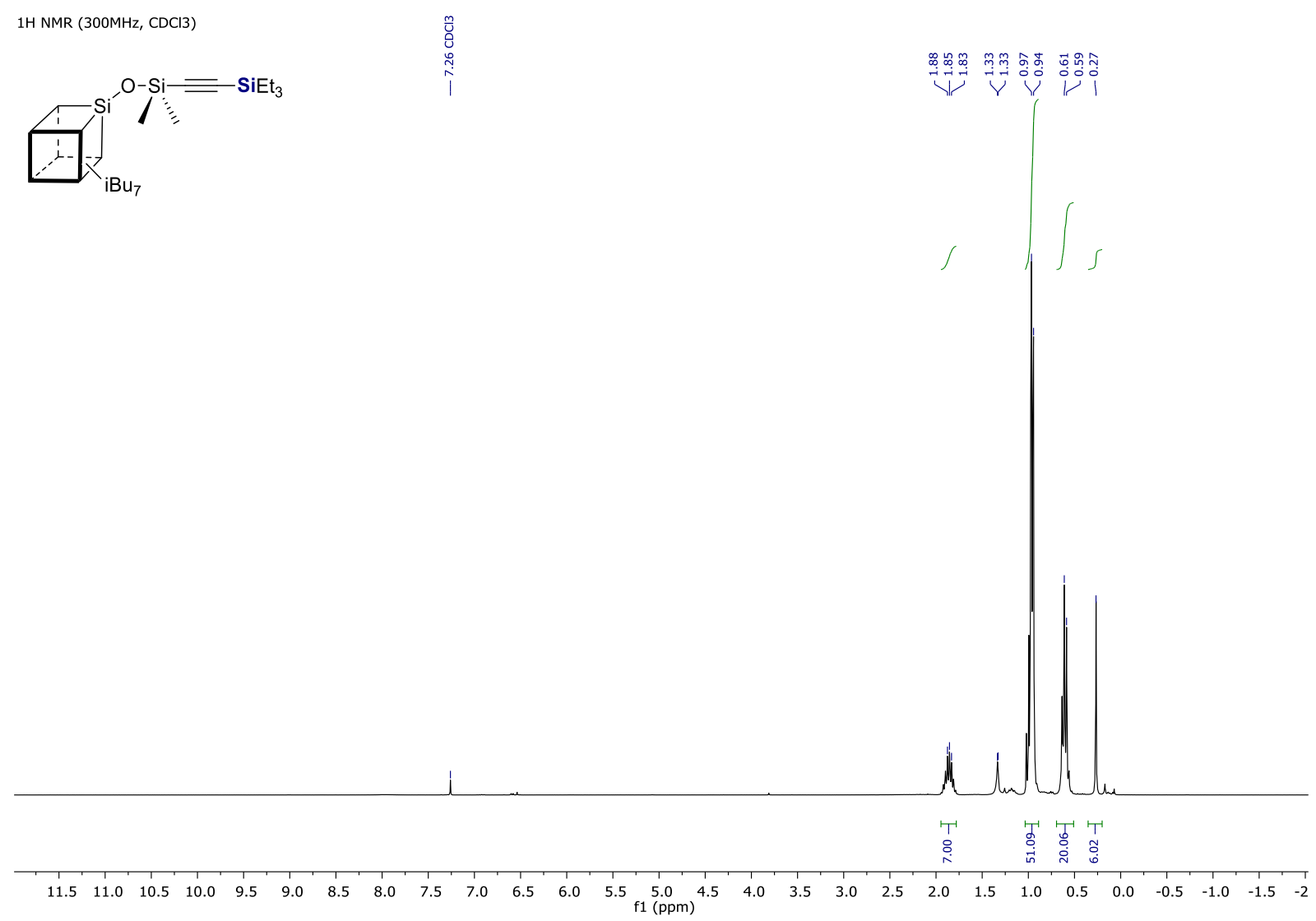

Figure S-12. ${ }^{1} \mathrm{H} N M R$ spectrum $\left(300 \mathrm{MHz}, \mathrm{CDCl}_{3}\right)$ of product $\mathrm{P}-1 \mathrm{C}$. 
13C NMR (100 MHZ, CDCl3)
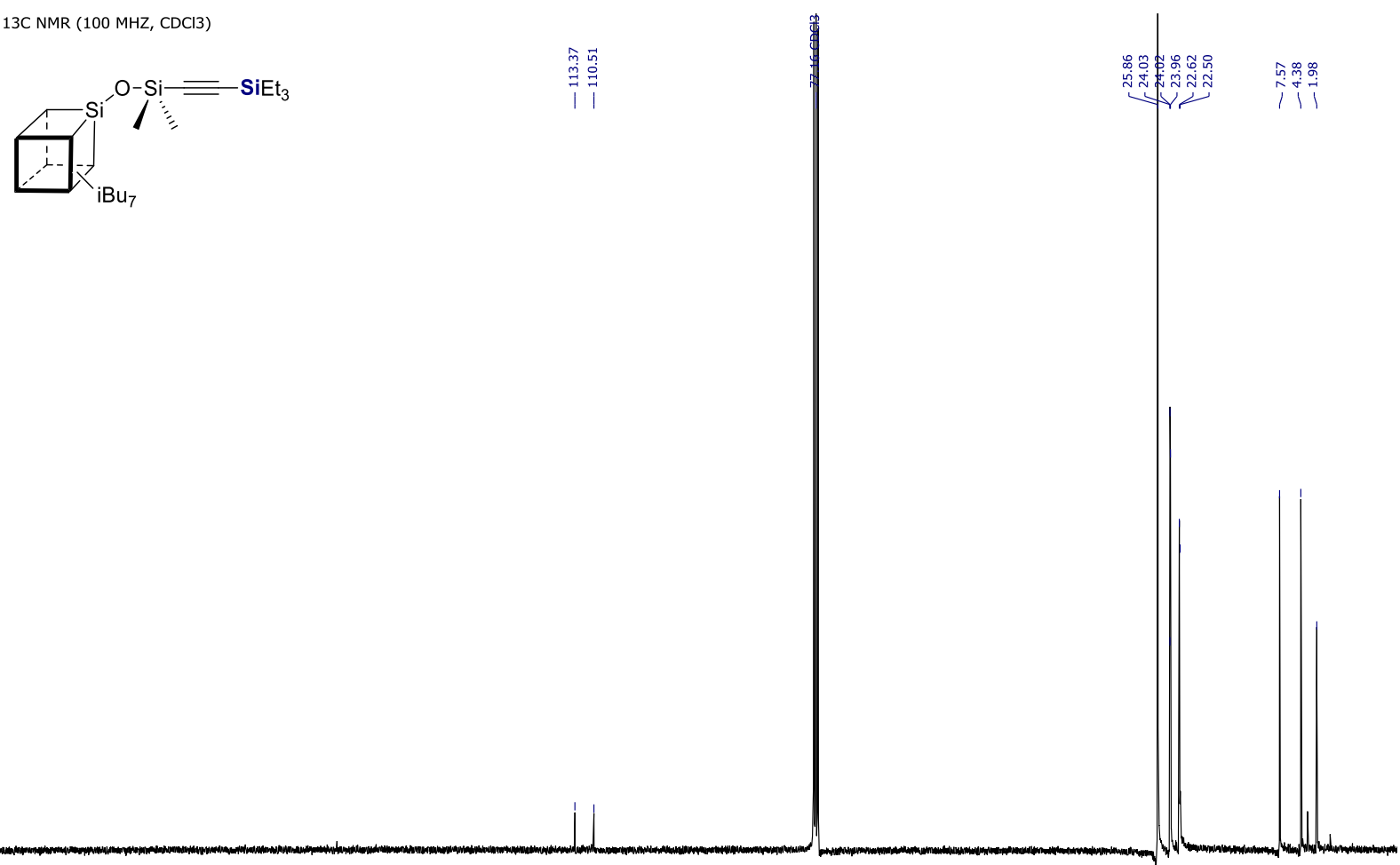

$\begin{array}{llllllllll}190 & 180 & 170 & 160 & 150 & 140 & 130 & 120 & 110 & 100 \\ \mathrm{f} 1(\mathrm{ppm})\end{array}$

Figure S-13. ${ }^{13} \mathrm{C}$ NMR spectrum $\left(100 \mathrm{MHz}, \mathrm{CDCl}_{3}\right)$ of product P-1c.

29Si NMR $(79 \mathrm{MHz}, \mathrm{CDCl} 3)$

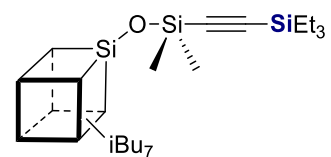

$\mathrm{iBu}_{7}$

$00 \quad 90$
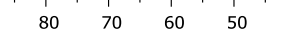

$\begin{array}{cr}-20 & -30 \\ \mathrm{f} 1(\mathrm{ppm})\end{array}$

$-40 \quad-50 \quad-60$

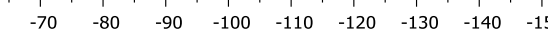

Figure S-14. ${ }^{29} \mathrm{Si} \mathrm{NMR}$ spectrum $\left(79 \mathrm{MHz}, \mathrm{CDCl}_{3}\right)$ of product $\mathrm{P}-1 \mathrm{c}$. 


\section{P-1d}

White solid. Isolated yield $70 \%$.

Product accompanied by homocoupling product of S-b, i.e. 1,2-bis(dimethylphenylsilyl)ethene (HP)

${ }^{1} \mathrm{H}$ NMR $\left(\mathrm{CDCl}_{3}, 400 \mathrm{MHz}\right): \delta$ 1.87-1.83 (m, 7H, iBu), 0.97-0.94 (m, 42H, iBu), 0.64-0.59 (m, 14H, iBu), $0.17\left(\mathrm{~s}, 3 \mathrm{H},-\mathrm{CH}_{3}(\mathrm{SiMe})\right), 0.14\left(\mathrm{~s}, 18 \mathrm{H},-\mathrm{CH}_{3}(\mathrm{OSiMe})_{2}\right), 0.07\left(\mathrm{~s}, 6 \mathrm{H},-\mathrm{SiMe}_{2}\right)$.

${ }^{13} \mathrm{C}$ NMR $\left(\mathrm{CDCl}_{3}, 75 \mathrm{MHz}\right): \delta$ 149.16(HP), $110.82(\mathrm{C} \equiv \mathrm{C}), 108.64(\mathrm{C} \equiv \mathrm{C}), 25.87,24.01,23.96,22.59$, 22.47, 2.01, 1.83, 1.67 .

${ }^{29}$ Si NMR ( $\left.\mathrm{CDCl}_{3}, 99 \mathrm{MHz}\right): \delta 10.12,8.02$ (HP), -17.31, -35.73 (HP), -53.81, -66.95, -67.89, -110.06.

EA: Anal. calcd for $\mathrm{C}_{39} \mathrm{H}_{90} \mathrm{O}_{15} \mathrm{Si}_{12}$ (\%):C, 41.23, $\mathrm{H}, 7.98$; found: $\mathrm{C}, 41.36 ; \mathrm{H}, 8.23$.
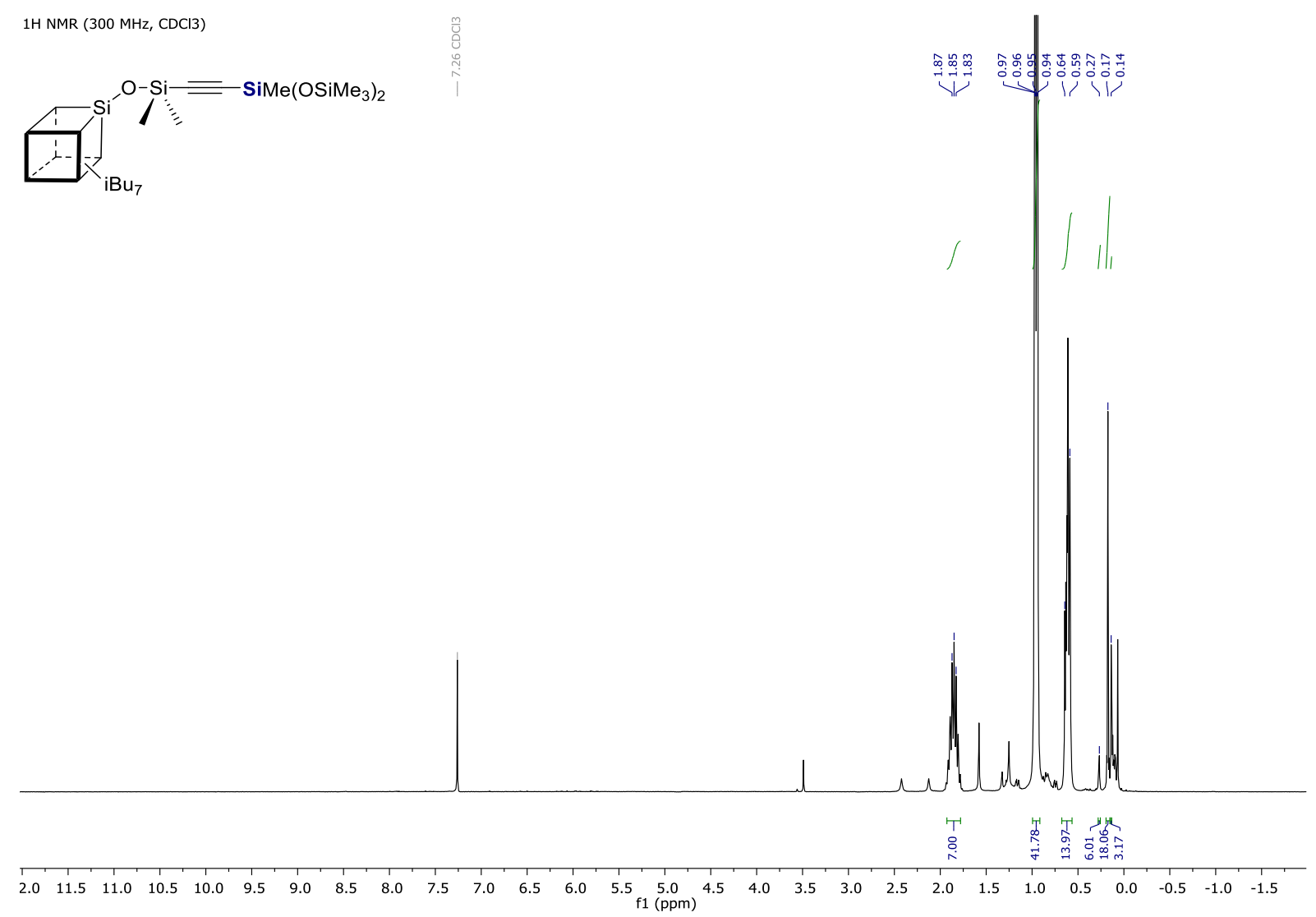

Figure S-15. ${ }^{1} \mathrm{H}$ NMR spectrum $\left(300 \mathrm{MHz}, \mathrm{CDCl}_{3}\right)$ of product $\mathrm{P}-1 \mathrm{~d}$. 


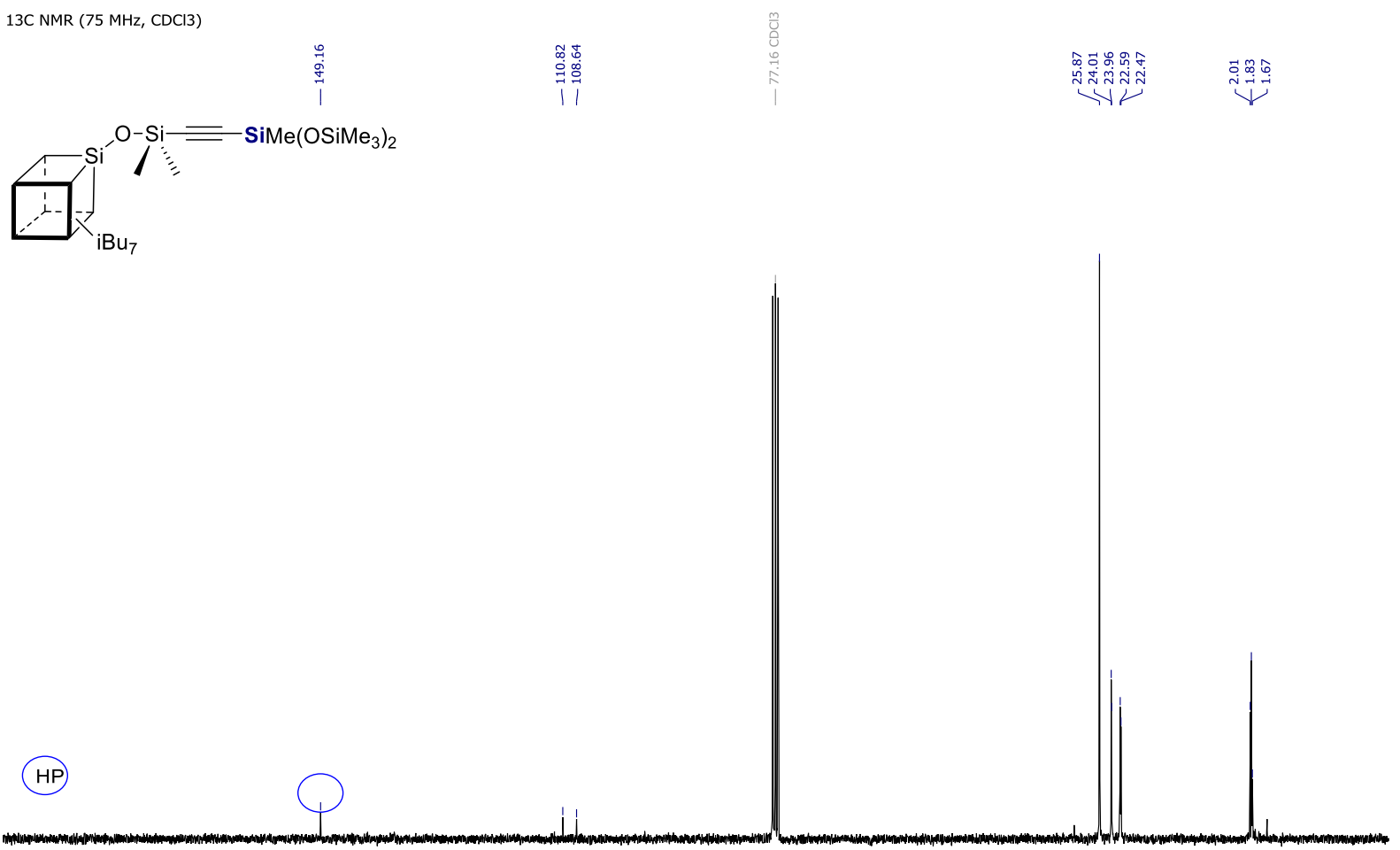

$\begin{array}{lllllllllllllllllllll}190 & 180 & 170 & 160 & 150 & 140 & 130 & 120 & 110 & 100 & \underset{\mathrm{f} 1}{90}(\mathrm{ppm}) & 80 & 70 & 60 & 50 & 40 & 30 & 20 & 10 & 0 & -10\end{array}$

Figure S-16. ${ }^{13} \mathrm{C}$ NMR spectrum $\left(75 \mathrm{MHz}, \mathrm{CDCl}_{3}\right)$ of product $\mathrm{P}-1 \mathrm{~d}$.

29Si NMR $(79 \mathrm{MHz}, \mathrm{CDCl} 3)$
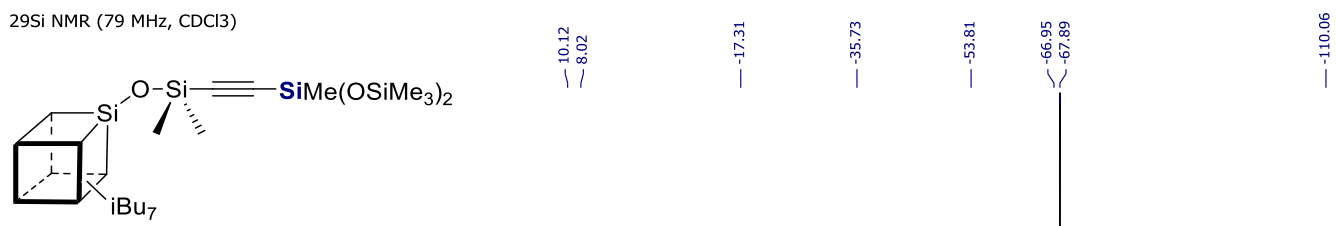

(HP)
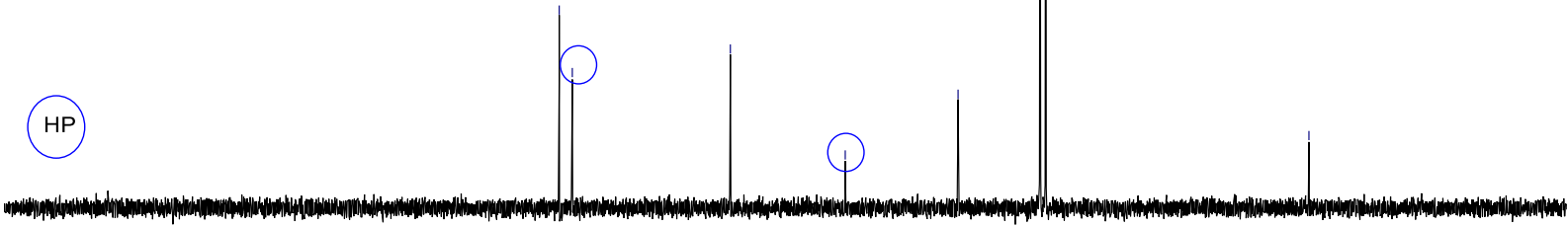

$\begin{array}{llllllllllllllllllllllllllll}190 & 80 & 70 & 60 & 50 & 40 & 30 & 20 & 10 & 0 & -10 & -20 & -30 & -40 & -50 & -60 & -70 & -80 & -90 & -100 & -110 & -120 & -130 & -140 & -15\end{array}$

Figure S-17. ${ }^{29} \mathrm{Si} \mathrm{NMR} \mathrm{spectrum}\left(79 \mathrm{MHz}, \mathrm{CDCl}_{3}\right)$ of product P-1d. 
White solid. Isolated yield $76 \%$.

${ }^{1} \mathrm{H}$ NMR $\left(\mathrm{CDCl}_{3}, 400 \mathrm{MHz}\right): \delta 1.00-0.96\left(\mathrm{~m}, 21 \mathrm{H},-\mathrm{CH}_{3}(\mathrm{Et})\right), 0.64-0.59\left(\mathrm{~m}, 14 \mathrm{H},-\mathrm{CH}_{2}-(\mathrm{Et})\right), 0.29(\mathrm{~s}, 6 \mathrm{H}$, $\left.-\mathrm{SiMe}_{2}\right), 0.17\left(\mathrm{~s}, 9 \mathrm{H},-\mathrm{SiMe}_{3}\right)$.

${ }^{13} \mathrm{C} \mathrm{NMR}\left(\mathrm{CDCl}_{3}, 100 \mathrm{MHz}\right): \delta 112.95(\mathrm{C} \equiv \mathrm{C}), 111.55(\mathrm{C} \equiv \mathrm{C}), 6.48,6.444 .04,3.97,1.70,-0.24$.

${ }^{29} \mathrm{Si}$ NMR $\left(\mathrm{CDCl}_{3}, 79 \mathrm{MHz}\right): \delta-17.15,-18.58,-65.06,-65.75,-108.93$.

EA: Anal. calcd for $\mathrm{C}_{21} \mathrm{H}_{50} \mathrm{O}_{13} \mathrm{Si}_{10}(\%): \mathrm{C}, 31.87, \mathrm{H}, 6.37$; found: $\mathrm{C}, 32.19 ; \mathrm{H}, 6.62$.

$1 \mathrm{H}$ NMR (300 MHz, CDCl3)
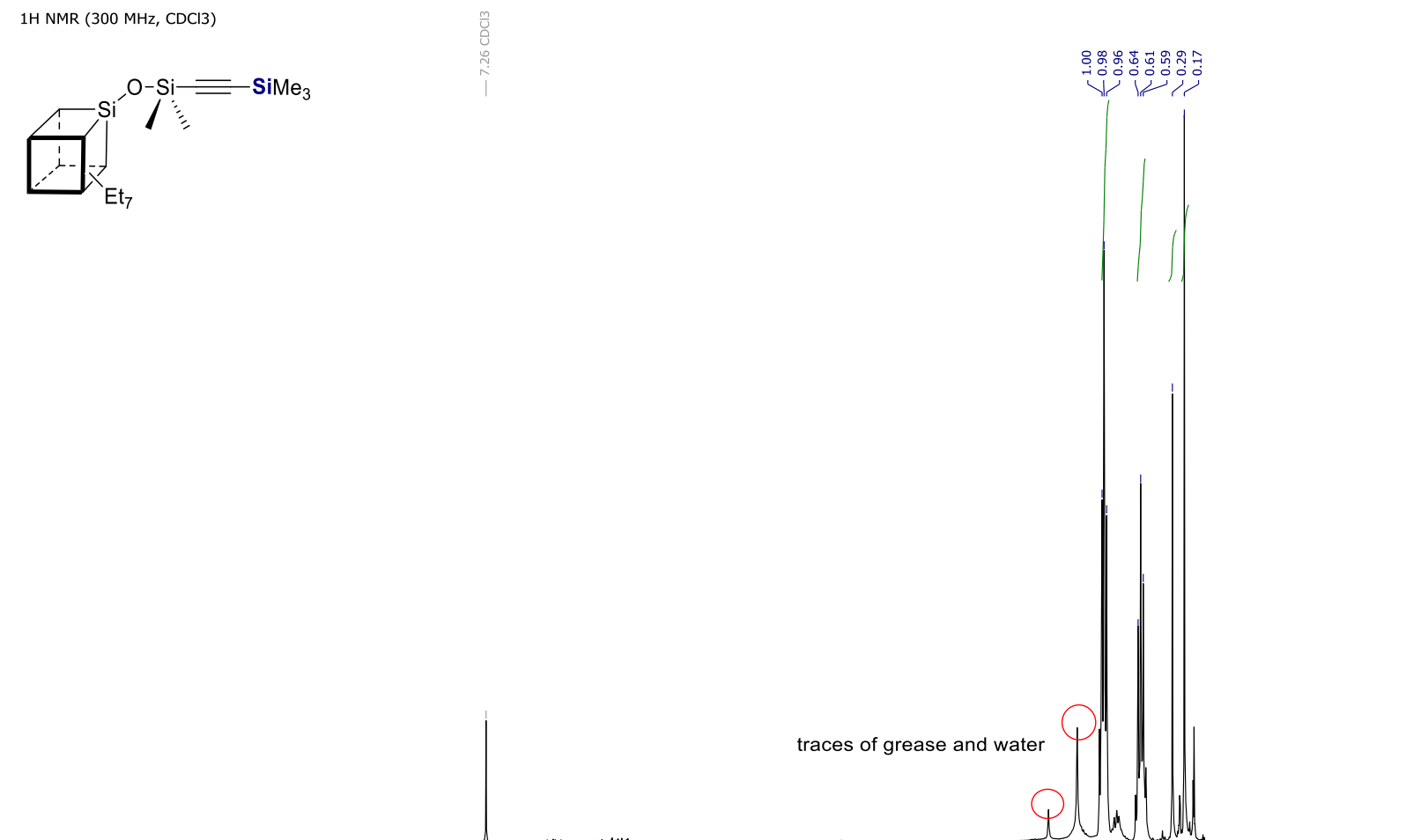

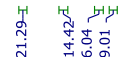

$\begin{array}{lllllllllllllllllllllllllllllll}2.0 & 11.5 & 11.0 & 10.5 & 10.0 & 9.5 & 9.0 & 8.5 & 8.0 & 7.5 & 7.0 & 6.5 & 6.0 & 1.5 & 5.0 & 4 & 4.5 & 4.0 & 3.5 & 3.0 & 2.5 & 2.0 & 1.5 & 1.0 & 0.5 & 0.0 & -0.5 & -1.0 & 1.5\end{array}$

Figure S-18. ${ }^{1} \mathrm{H}$ NMR spectrum $\left(300 \mathrm{MHz}, \mathrm{CDCl}_{3}\right)$ of product $\mathrm{P}-2 \mathrm{a}$. 
$\begin{array}{llllllllll}190 & 180 & 170 & 160 & 150 & 140 & 130 & 120 & 110 & \begin{array}{l}100 \\ \mathrm{f} 1(\mathrm{ppm})\end{array}\end{array}$

Figure S-19. ${ }^{13} \mathrm{C} \mathrm{NMR}$ spectrum $\left(100 \mathrm{MHz}, \mathrm{CDCl}_{3}\right)$ of product $\mathrm{P}$-2a.
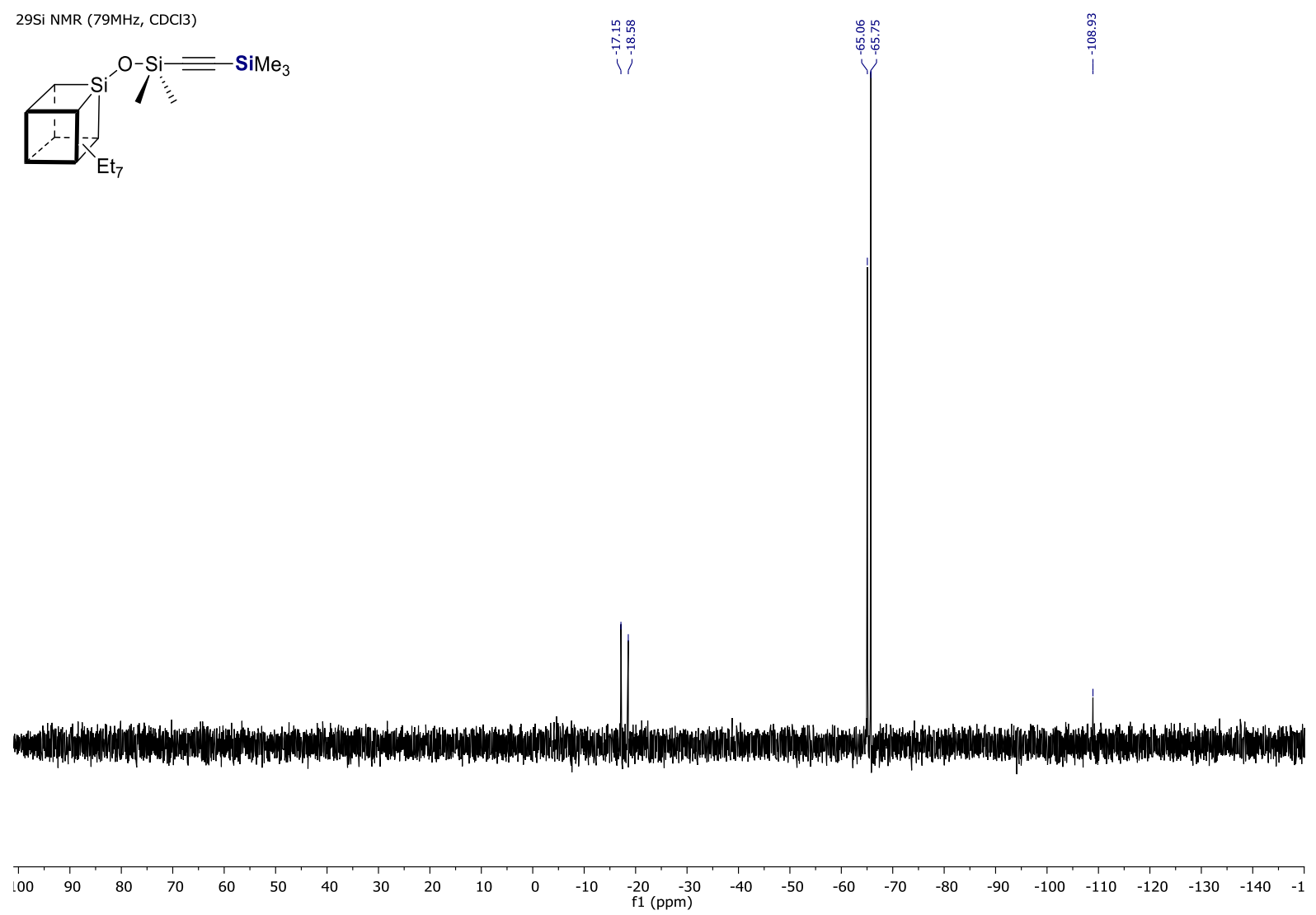

Figure S-20. ${ }^{29} \mathrm{Si} \mathrm{NMR} \mathrm{spectrum} \mathrm{(79} \mathrm{MHz,} \mathrm{CDCl}_{3}$ ) of product P-2a. 


\section{$\mathbf{P}-\mathbf{2 b}$}

White solid. Isolated yield $94 \%$.

Product accompanied by homocoupling product of S-b, i.e. 1,2-bis(dimethylphenylsilyl)ethene (HP)

${ }^{1} \mathrm{H}_{\mathrm{NMR}}\left(\mathrm{CDCl}_{3}, 300 \mathrm{MHz}\right): \delta$ 7.65-7.59 (m, 2H, Ph), 7.53-7.48 (m, 3H, Ph), $6.81(\mathrm{HP}), 1.01-0.96(\mathrm{~m}$, $\left.21 \mathrm{H},-\mathrm{CH}_{3}(\mathrm{Et})\right), 0.65-0.54\left(\mathrm{~m}, 14 \mathrm{H}-\mathrm{CH}_{2^{-}}(\mathrm{Et})\right), 0.41\left(\mathrm{~s}, 6 \mathrm{H}-\mathrm{SiMe}_{2} \mathrm{Ph}\right) 0.34(\mathrm{HP}), 0.30$ (s, 6H -SiMe $)$.

${ }^{13} \mathrm{C}$ NMR $\left(\mathrm{CDCl}_{3}, 75 \mathrm{MHz}\right): \delta 150.47$ (HP), 138.69 (HP), 134.04 (HP), 136.88, 133.86, 129.56, 127.99, 127.91 (HP), 113.77 ( $\mathrm{C} \equiv \mathrm{C}), 110.68$ ( $\mathrm{C} \equiv \mathrm{C}), 6.67,6.62,4.19,4.14,1.85,-0.81,-2.77$ (HP)

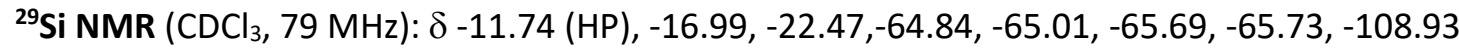

EA: Anal. calcd for $\mathrm{C}_{26} \mathrm{H}_{52} \mathrm{O}_{13} \mathrm{Si}_{10}$ (\%):C, 36.59, $\mathrm{H}, 6.14$; found: $\mathrm{C}, 36.96 ; \mathrm{H}, 6.41$.

$1 \mathrm{H}$ NMR $(300 \mathrm{MHz}, \mathrm{CDCl} 3)$
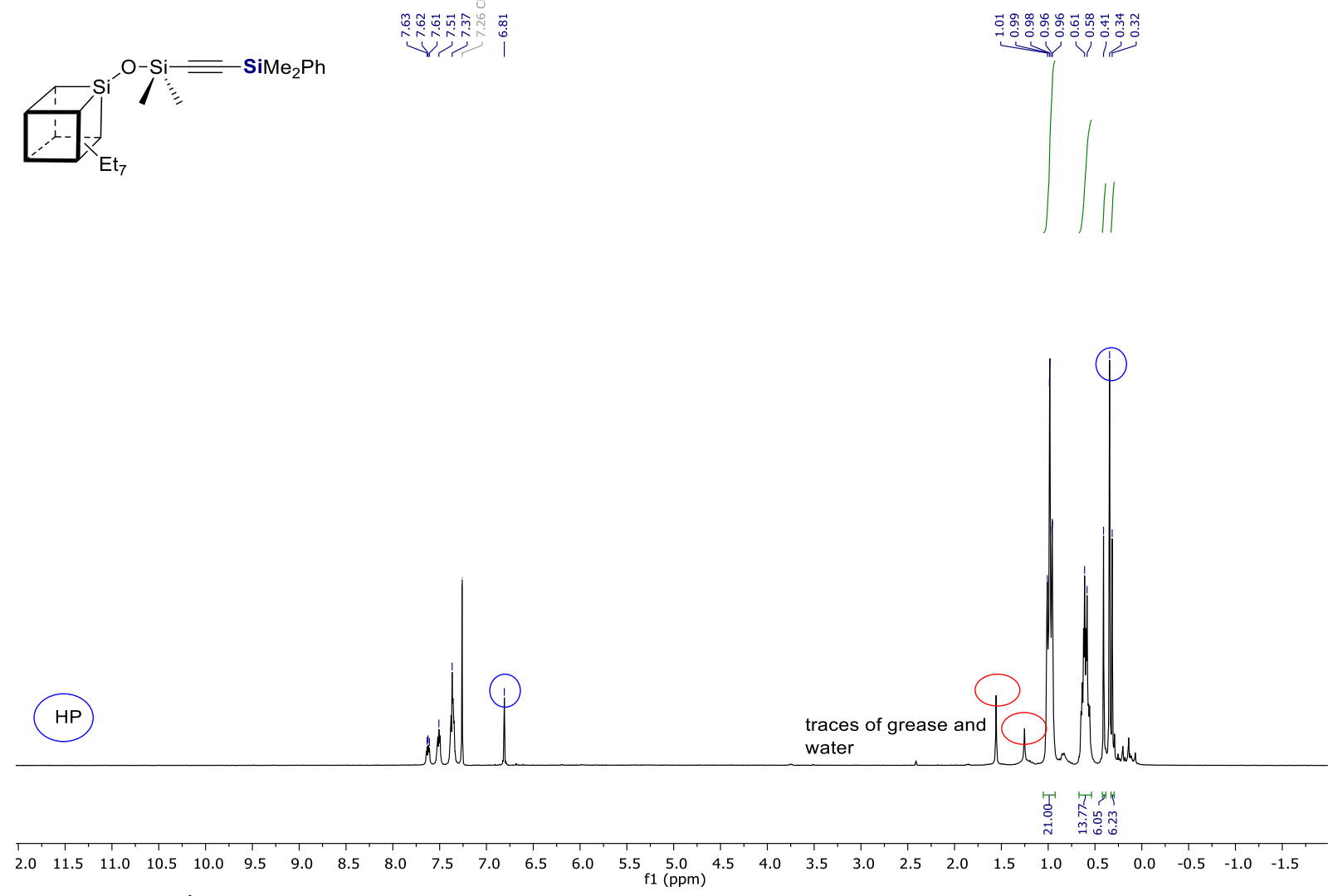

Figure S-21. ${ }^{1} \mathrm{H}$ NMR spectrum $\left(300 \mathrm{MHz}, \mathrm{CDCl}_{3}\right.$ ) of product $\mathrm{P}-2 \mathrm{~b}$. 


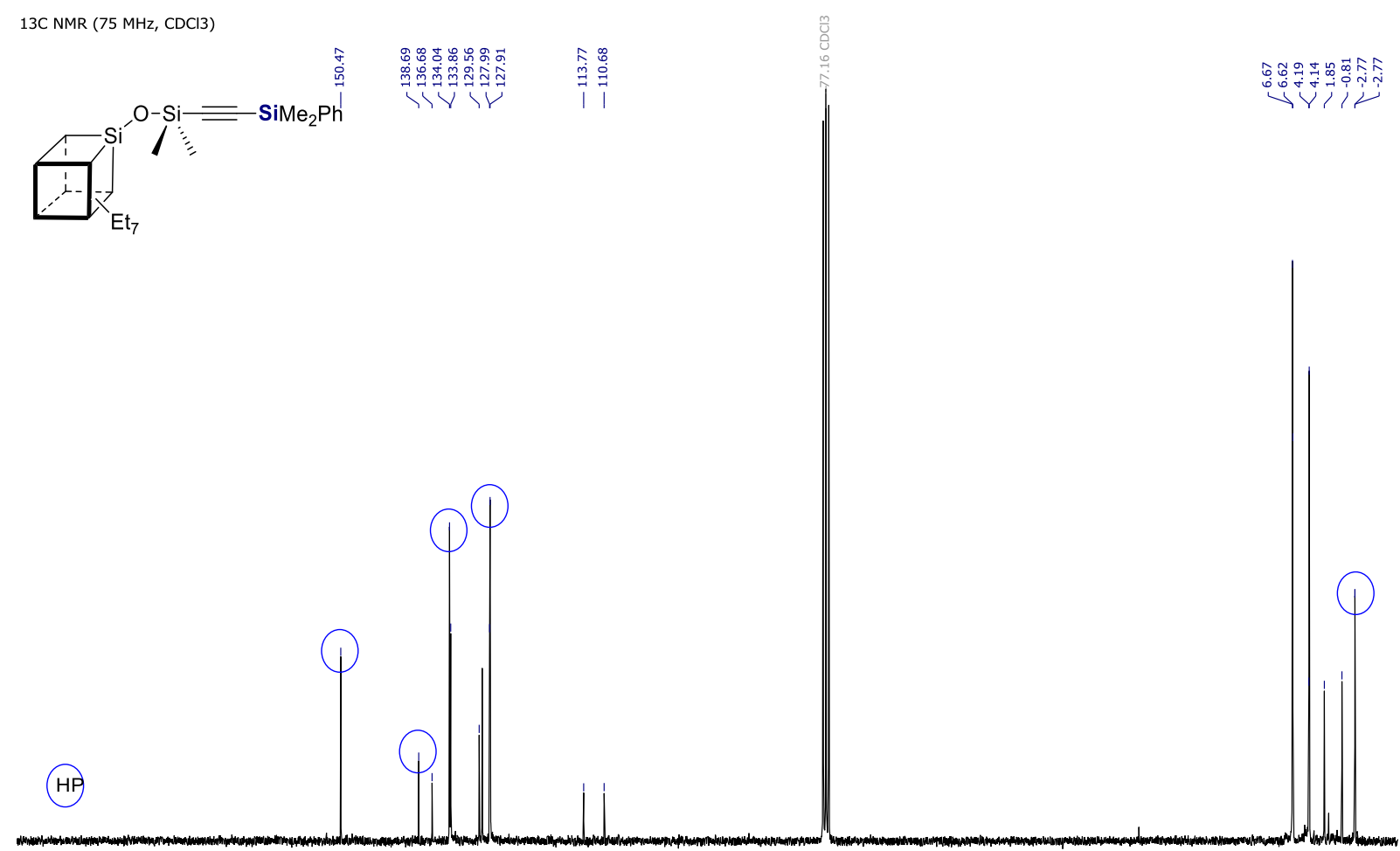

$\begin{array}{llllllllllll}190 & 180 & 170 & 160 & 150 & 140 & 130 & 120 & 110 & \begin{array}{c}100 \\ \mathrm{f} 1(\mathrm{ppm})\end{array} & 80 & \end{array}$

Figure S-22. ${ }^{13} \mathrm{C} N M R$ spectrum $\left(75 \mathrm{MHz}, \mathrm{CDCl}_{3}\right)$ of product $\mathrm{P}-2 \mathrm{~b}$.

29Si NMR $(79 \mathrm{MHz}, \mathrm{CDCl} 3)$

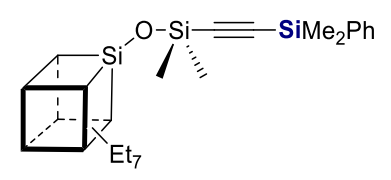

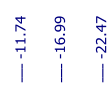

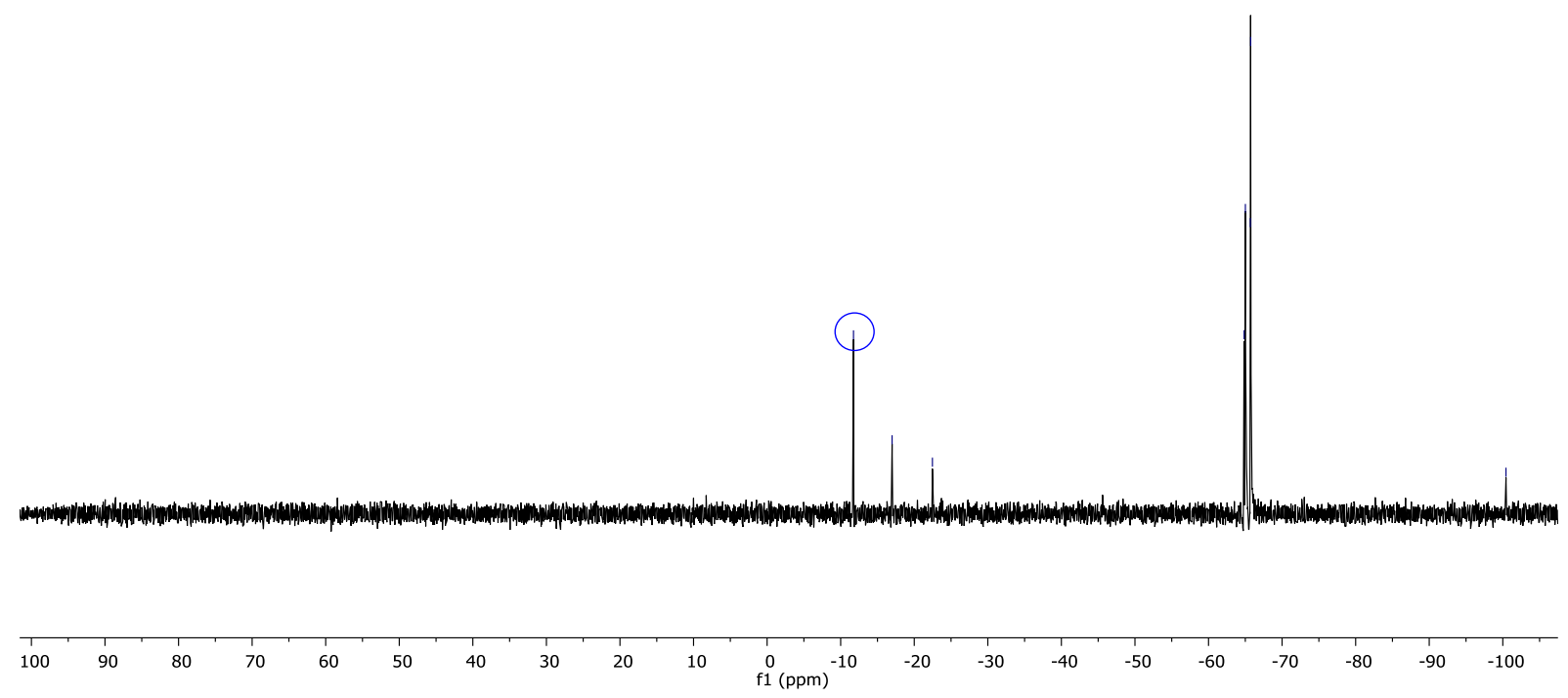

Figure S-23. ${ }^{29} \mathrm{Si} \mathrm{NMR} \mathrm{spectrum}\left(79 \mathrm{MHz}, \mathrm{CDCl}_{3}\right)$ of product $\mathrm{P}-2 \mathrm{~b}$. 


\section{P-2c}

White solid. Isolated yield $76 \%$.

${ }^{1} \mathrm{H} \mathrm{NMR}\left(\mathrm{CDCl}_{3}, 300 \mathrm{MHz}\right): \delta 1.03-0.96(\mathrm{~m}, 30 \mathrm{H}, \mathrm{Et}), 0.63-0.56(\mathrm{~m}, 2 \mathrm{H}, \mathrm{Et}), 0.29\left(\mathrm{~s}, 6 \mathrm{H},-\mathrm{SiMe}_{2}\right)$.

${ }^{13} \mathrm{C} \mathrm{NMR}\left(\mathrm{CDCl}_{3}, 100 \mathrm{MHz}\right): \delta 113.28(\mathrm{C} \equiv \mathrm{C}), 110.68(\mathrm{C} \equiv \mathrm{C}), 7.52,6.63,6.58,4.43,4.13,4.11,1.95$.

${ }^{29} \mathrm{Si} \mathrm{NMR}\left(\mathrm{CDCl}_{3}, 79 \mathrm{MHz}\right): \delta-7.88,-17.43,-65.07,-65.76,-109.05$.

EA: Anal. calcd for $\mathrm{C}_{24} \mathrm{H}_{56} \mathrm{O}_{13} \mathrm{Si}_{10}$ (\%):C, 34.58, $\mathrm{H}, 6.77$; found: $\mathrm{C}, 34.72 ; \mathrm{H}, 6.89$.

$1 \mathrm{H}$ NMR (300MHz, CDCl3)
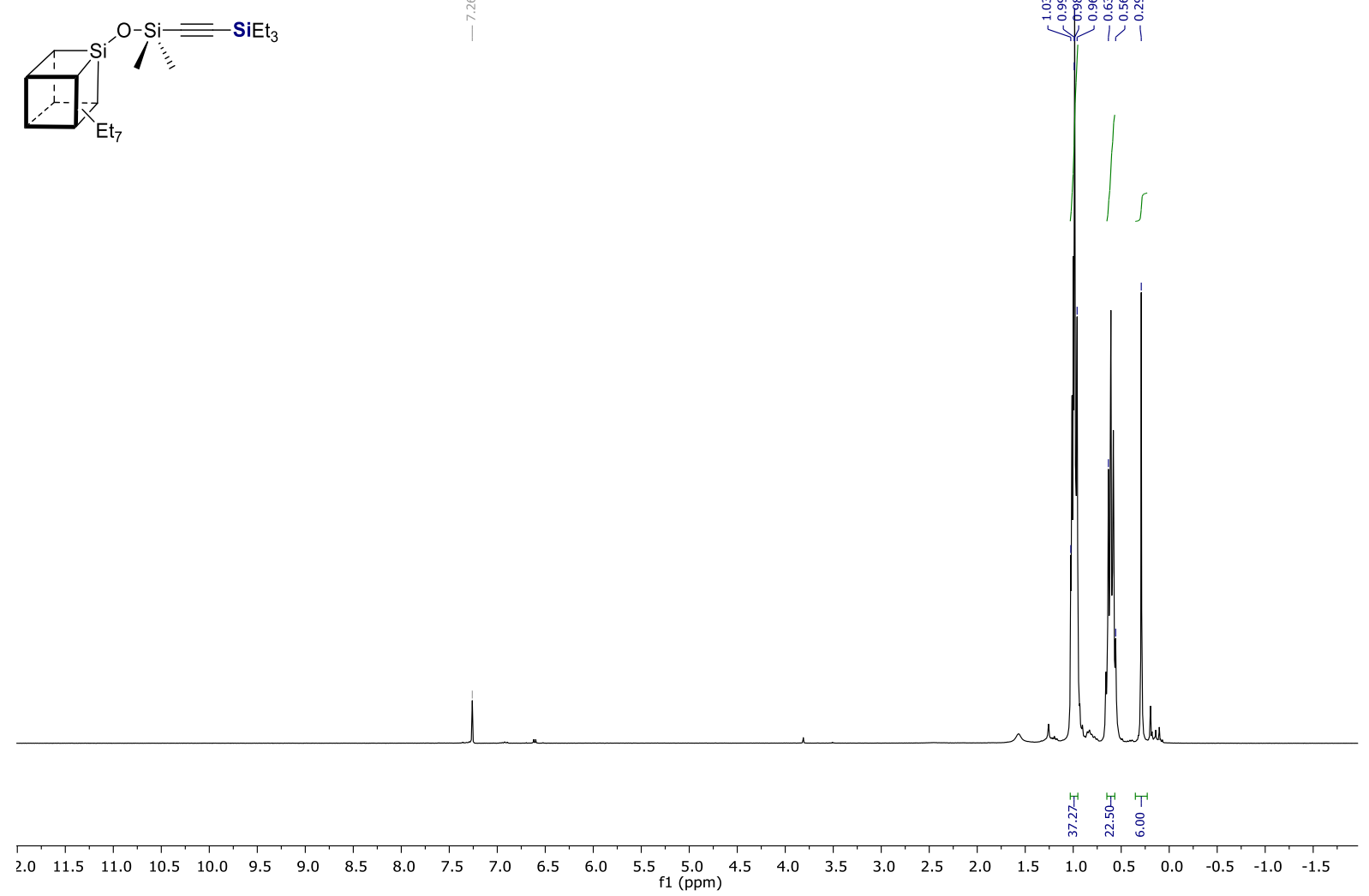

Figure S-24. ${ }^{1} \mathrm{H}$ NMR spectrum $\left(300 \mathrm{MHz}, \mathrm{CDCl}_{3}\right)$ of product $\mathrm{P}-2 \mathrm{c}$. 
13C NMR (100 MHz, CDCl3)
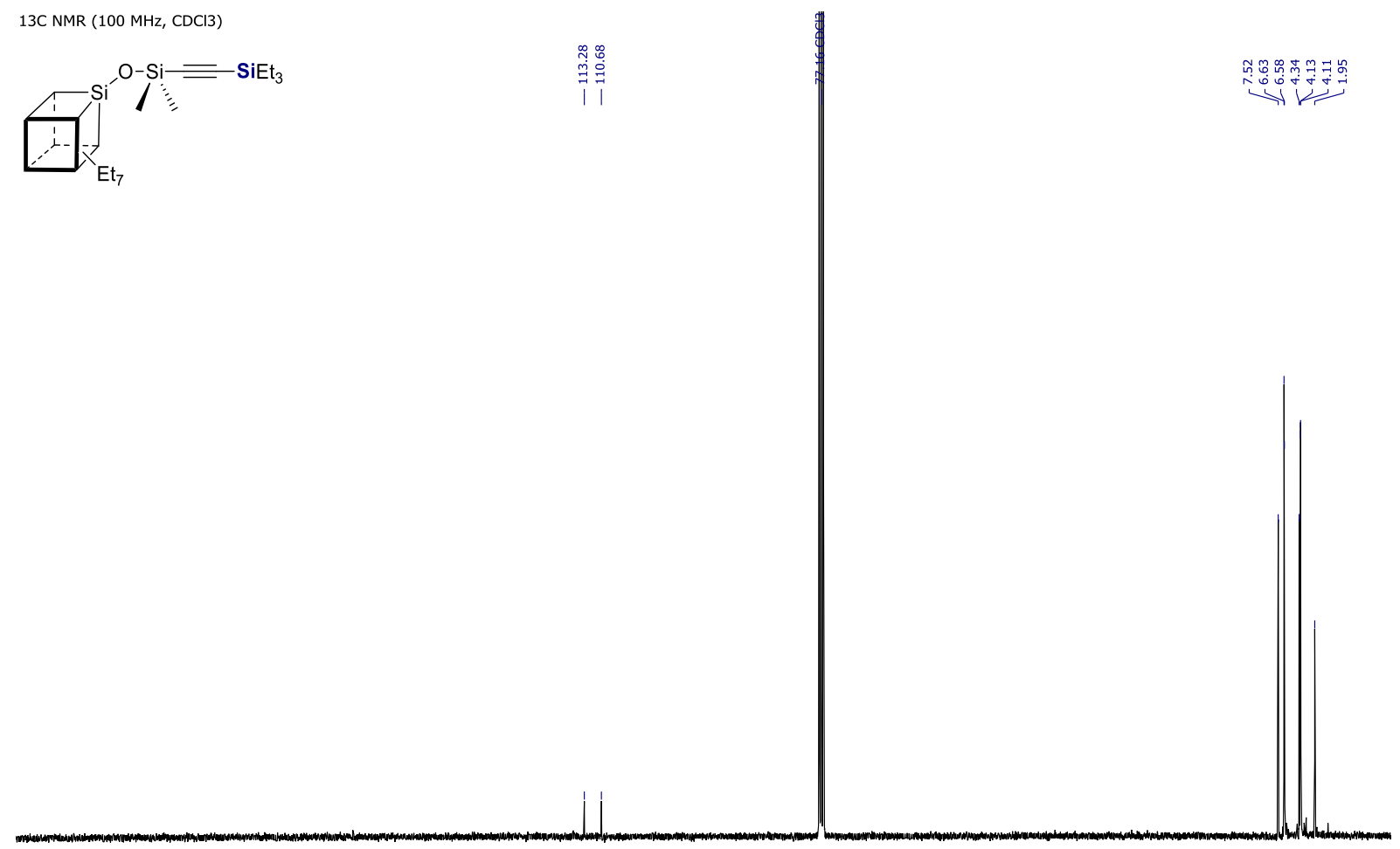

Figure S-25. ${ }^{13} \mathrm{C}$ NMR spectrum $\left(100 \mathrm{MHz}, \mathrm{CDCl}_{3}\right)$ of product $\mathrm{P}-2 \mathrm{c}$.

29Si NMR (79 MHz, CDCl3)

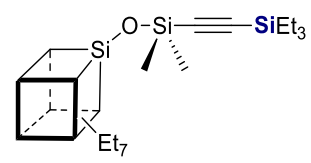

$\mathrm{Et}_{7}$

$00 \quad 90$

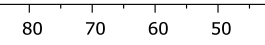

$\begin{array}{lll}40 & 30 \quad 20\end{array}$

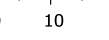

$\mathrm{f}_{1}^{-20}(\mathrm{ppm})$

$-40-50$

Figure S-26. ${ }^{29} \mathrm{Si} \mathrm{NMR}$ spectrum $\left(79 \mathrm{MHz}, \mathrm{CDCl}_{3}\right)$ of product $\mathrm{P}-2 \mathrm{c}$. 


\section{P-2d}

White solid. Isolated yield $80 \%$.

${ }^{1} \mathrm{H}$ NMR $\left(\mathrm{CDCl}_{3}, 300 \mathrm{MHz}\right): \delta 1.00-0.98(\mathrm{~m}, 21 \mathrm{H}, \mathrm{Et}), 0.63-0.60(\mathrm{~m}, 14 \mathrm{H}, \mathrm{Et}), 0.29(\mathrm{~s}, 6 \mathrm{H}), 0.15(\mathrm{~s}, 3 \mathrm{H}$ $\left.\mathrm{SiMe}\left(\mathrm{OSiMe}_{3}\right)_{2}\right), 0.13\left(\mathrm{~s}, 18 \mathrm{H}, \mathrm{SiMe}\left(\mathrm{OSiMe}_{3}\right)_{2}\right)$

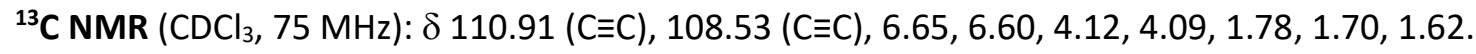

${ }^{29} \mathrm{Si} \mathrm{NMR}\left(\mathrm{CDCl}_{3}, 99 \mathrm{MHz}\right): \delta 10.14,-16.91,-53.85,-65.05,-65.15,-65.77,-109.07$.

EA: Anal. calcd for $\mathrm{C}_{25} \mathrm{H}_{62} \mathrm{O}_{15} \mathrm{Si}_{12}$ (\%):C, 31.95, $\mathrm{H}, 6.65$; found: $\mathrm{C}, 32.04 ; \mathrm{H}, 6.71$.
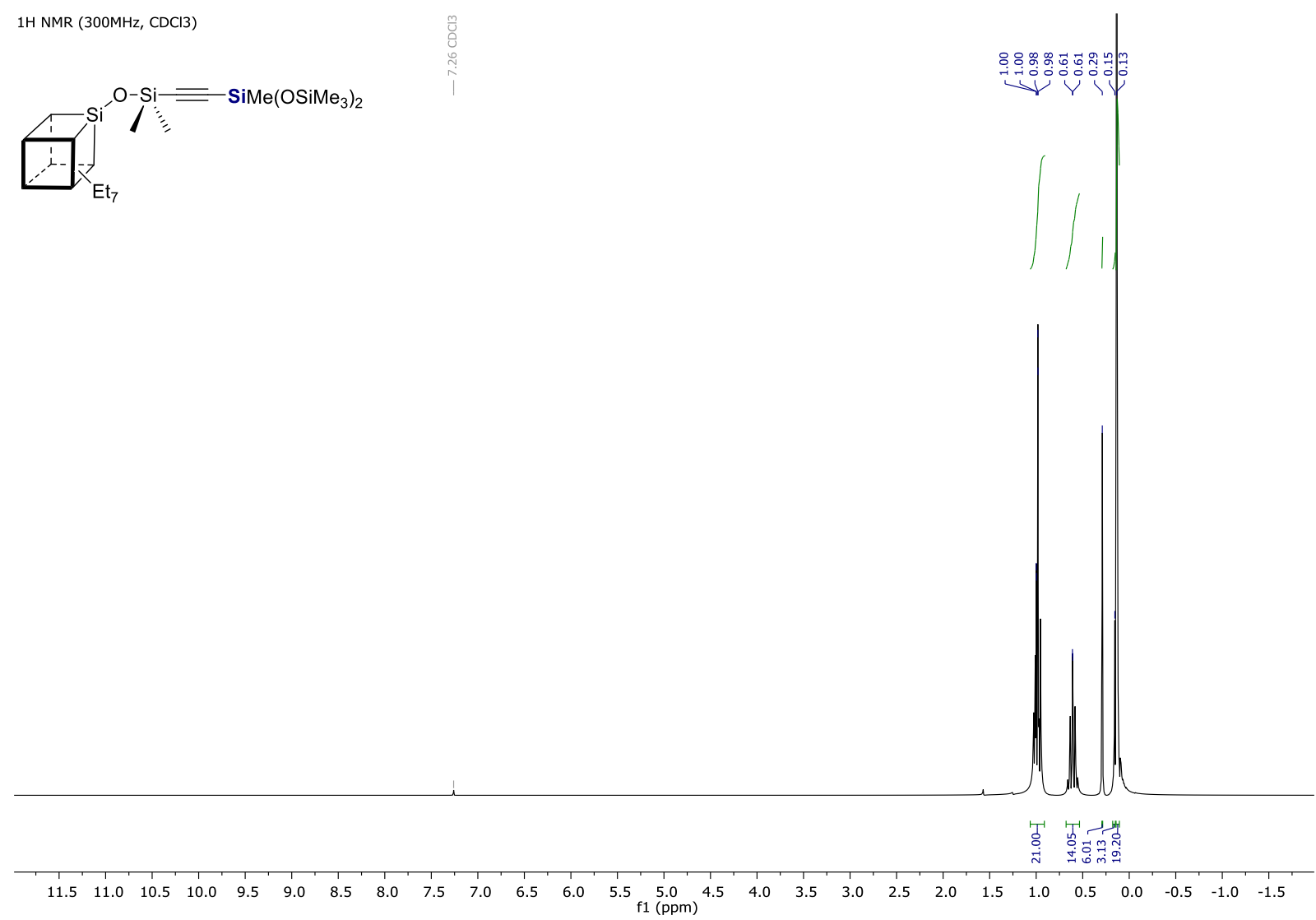

Figure S-27. ${ }^{1} \mathrm{H}$ NMR spectrum $\left(100 \mathrm{MHz}, \mathrm{CDCl}_{3}\right)$ of product $\mathrm{P}-2 \mathrm{~d}$. 


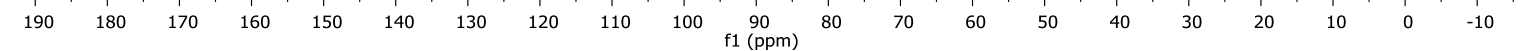

Figure S-28. ${ }^{13} \mathrm{C}$ NMR spectrum $\left(75 \mathrm{MHz}, \mathrm{CDCl}_{3}\right)$ of product P-2d.
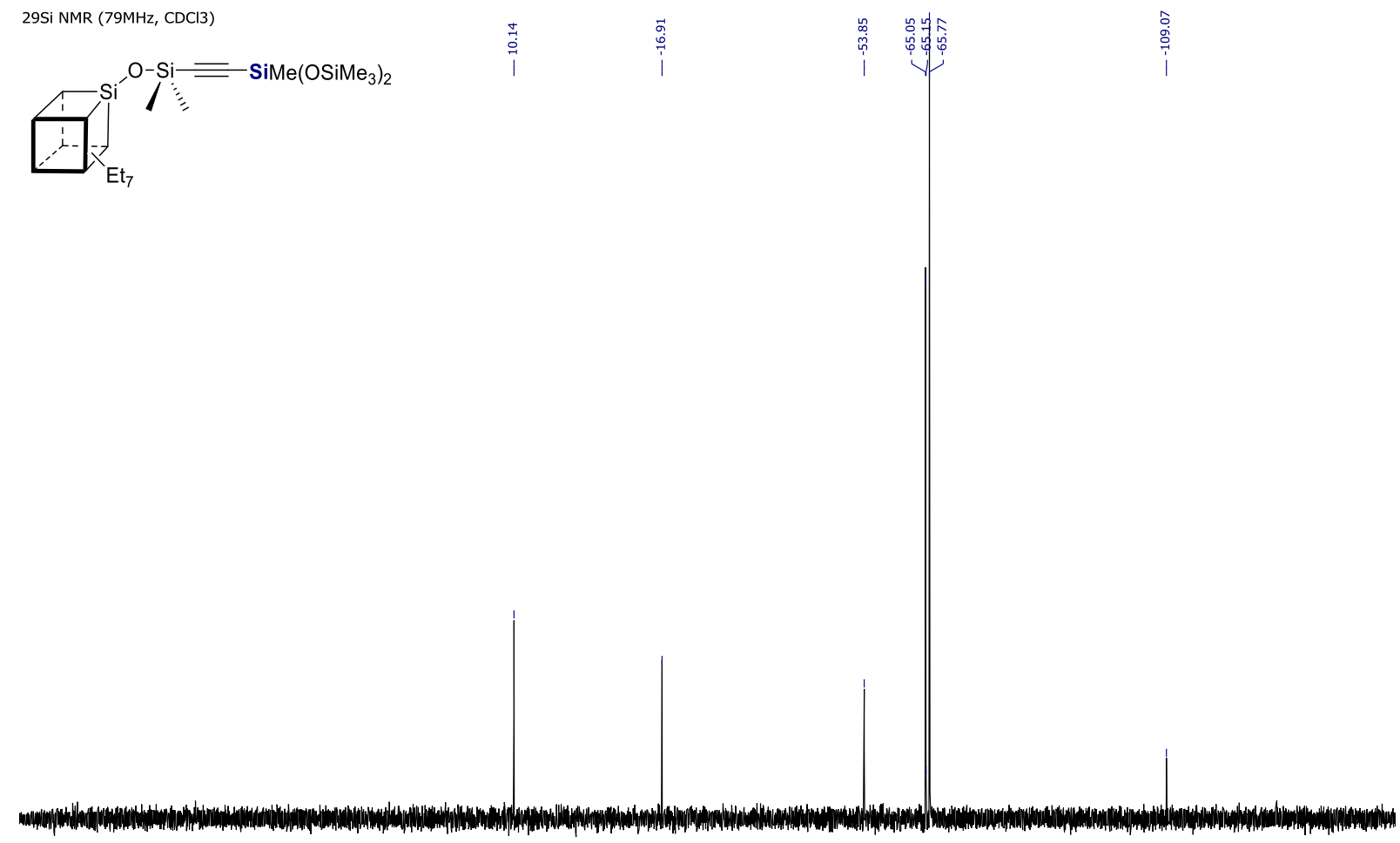

$\begin{array}{llllllllllllllllllllllllllll}00 & 90 & 80 & 70 & 60 & 50 & 40 & 30 & 20 & 10 & 0 & -10 & -20 & -30 & -40 & -50 & -60 & -70 & -80 & -90 & -100 & -110 & -120 & -130 & -140 & -1 !\end{array}$

Figure S-29. ${ }^{29} \mathrm{Si} \mathrm{NMR} \mathrm{spectrum}\left(79 \mathrm{MHz}, \mathrm{CDCl}_{3}\right)$ of product $\mathrm{P}-2 \mathrm{~d}$. 
White solid. Isolated yield $90 \%$.

${ }^{1} \mathrm{H}$ NMR $\left(\mathrm{CDCl}_{3}, 300 \mathrm{MHz}\right): \delta 1.75-1.49(\mathrm{~m}, 56 \mathrm{H}, \mathrm{Cyp}), 1.00-0.89$ (m, 7H, Cyp), $0.28\left(\mathrm{~s}, 6 \mathrm{H},-\mathrm{SiMe}_{2}\right)$, $0.17\left(\mathrm{~s}, 9 \mathrm{H},-\mathrm{SiMe}_{3}\right)$.

${ }^{13} \mathrm{C} \mathrm{NMR}\left(\mathrm{CDCl}_{3}, 75 \mathrm{MHz}\right): \delta 112.88(\mathrm{C} \equiv \mathrm{C}), 111.81(\mathrm{C} \equiv \mathrm{C}), 27.43,27.16,22.31,22.22,1.94,-0.08$.

${ }^{29} \mathrm{Si} N \mathrm{NMR}\left(\mathrm{CDCl}_{3}, 79 \mathrm{MHz}\right): \delta-17.52,-18.64,-65.93,-66.55,-108.68$

EA: Anal. calcd for $\mathrm{C}_{42} \mathrm{H}_{78} \mathrm{O}_{13} \mathrm{Si}_{10}$ (\%):C, 47.06, $\mathrm{H}, 7.33$; found: $\mathrm{C}, 47.32 ; \mathrm{H}, 7.63$.
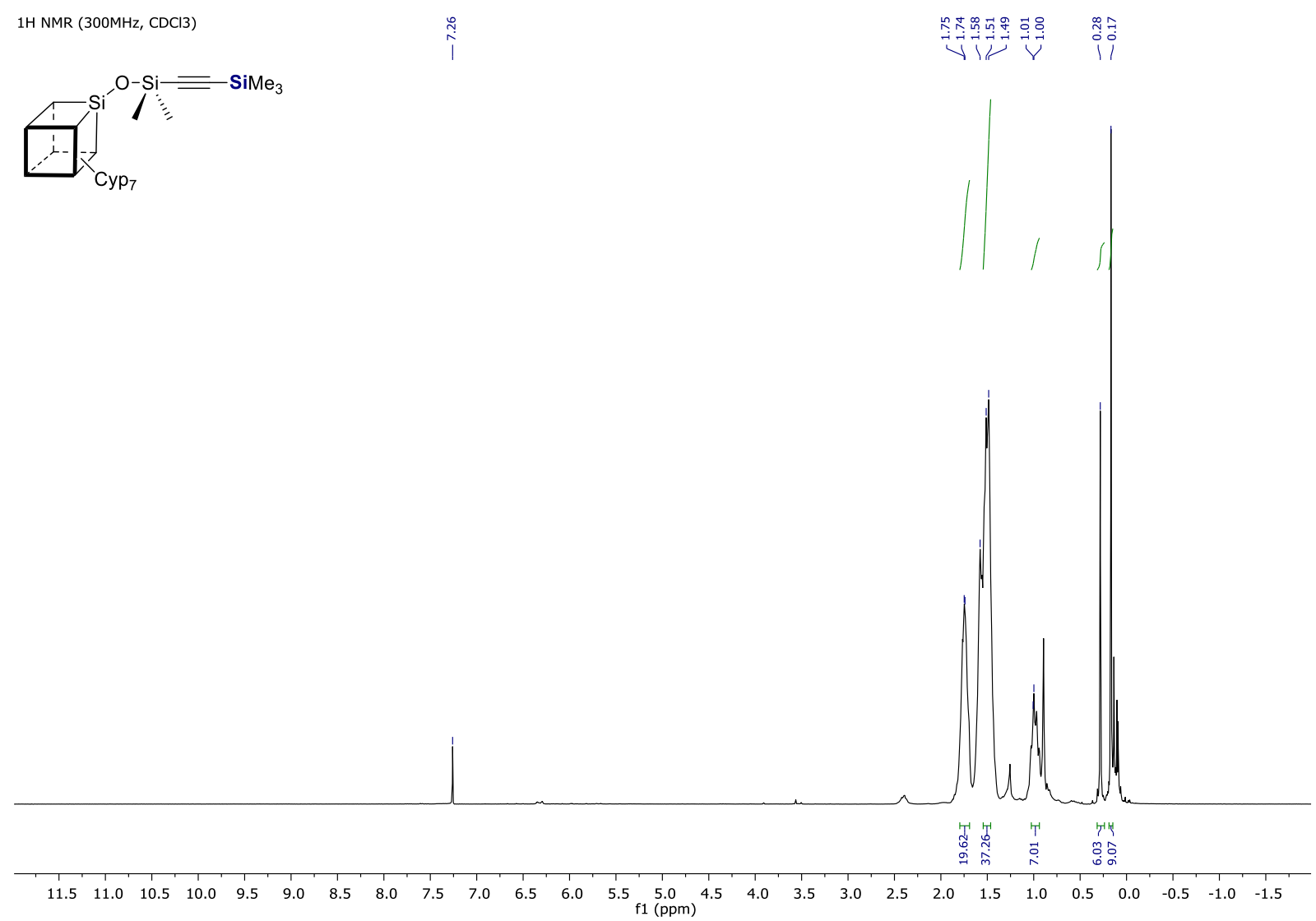

Figure S-30. ${ }^{1} \mathrm{H}$ NMR spectrum ( $300 \mathrm{MHz}, \mathrm{CDCl}_{3}$ ) of product $\mathrm{P}-3 \mathrm{a}$. 


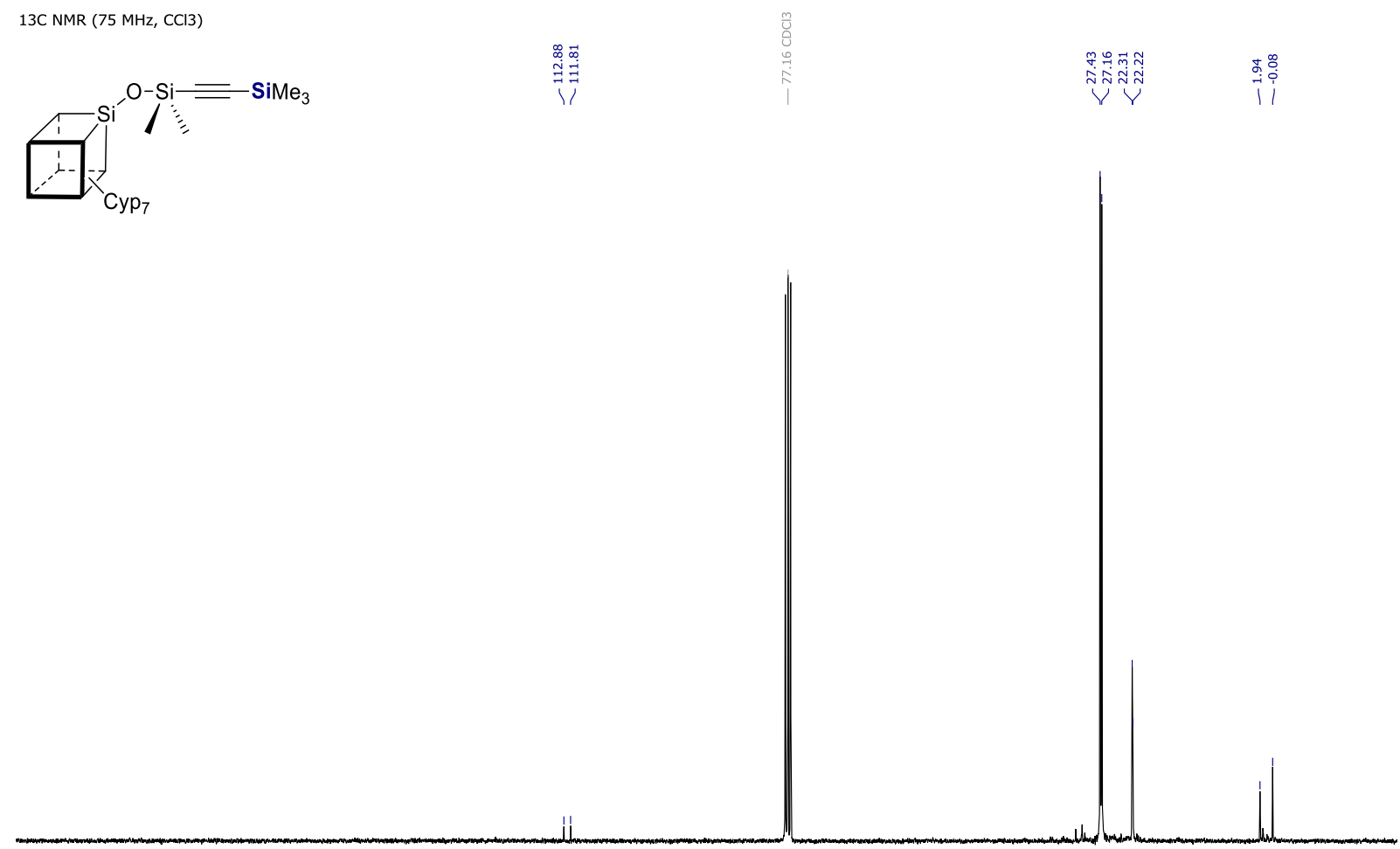

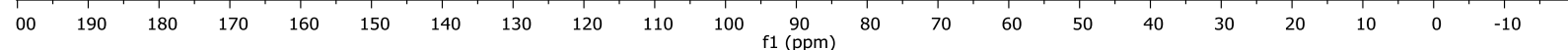

Figure S-31. ${ }^{13} \mathrm{C}$ NMR spectrum $\left(75 \mathrm{MHz}, \mathrm{CDCl}_{3}\right)$ of product P-3a.

29Si NMR (79 MHz, CDCl3)
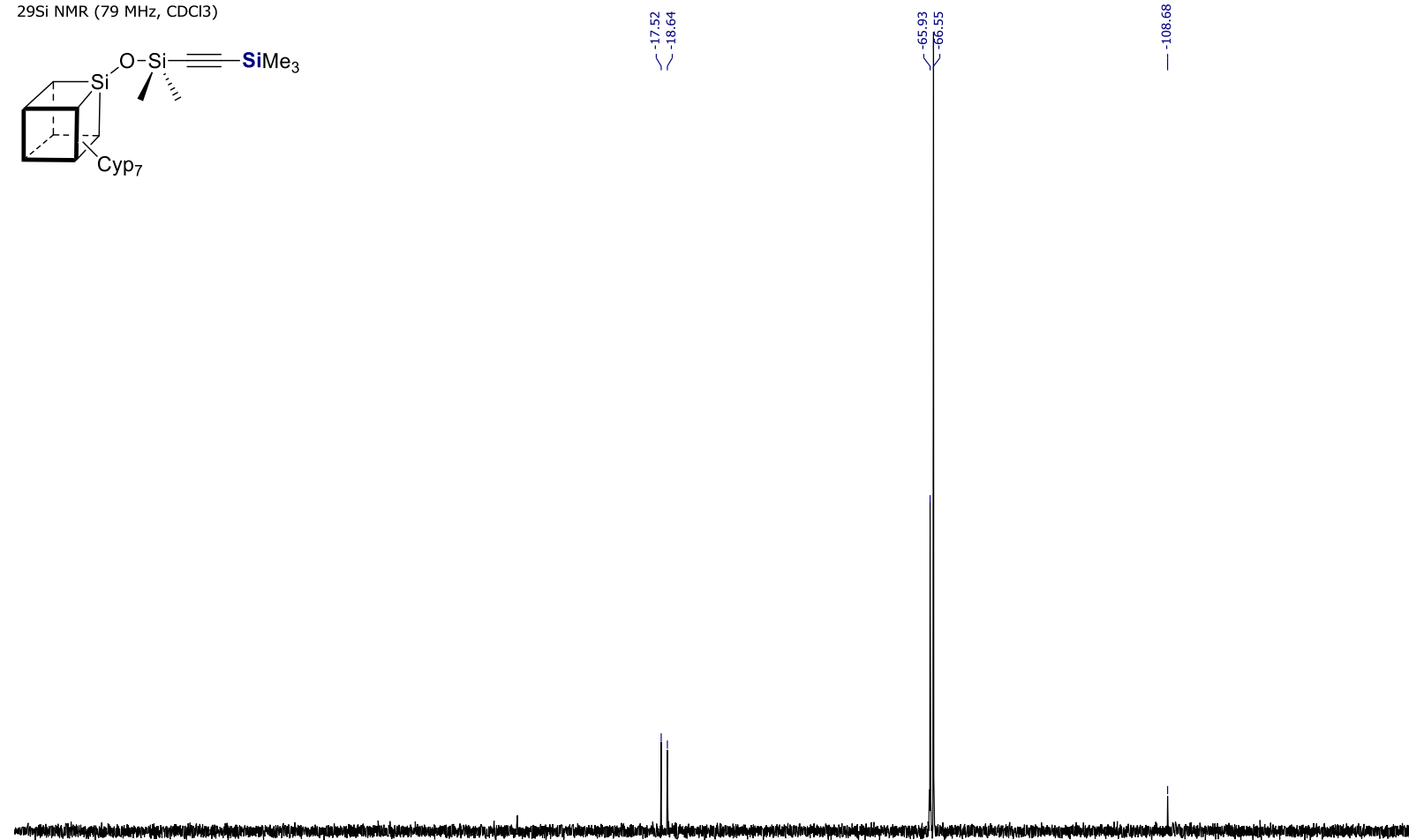

$\begin{array}{llllllllllllllllllllllllllll}90 & 80 & 70 & 60 & 50 & 40 & 30 & 20 & 10 & 0 & -10 & -20 & -30 & -40 & -50 & -60 & -70 & -80 & -90 & -100 & -110 & -120 & -130 & -140 & -15 & \end{array}$ Figure S-32. ${ }^{29} \mathrm{Si} \mathrm{NMR}$ spectrum $\left(79 \mathrm{MHz}, \mathrm{CDCl}_{3}\right)$ of product P-3a. 
White solid. Isolated yield $60 \%$.

${ }^{1} \mathrm{H}$ NMR ( $\left.\mathrm{CDCl}_{3}, 300 \mathrm{MHz}\right): \delta$ 7.64-7.61 (m, 2H, Ph), 7.38-7.36 (m, 3H, Ph), $1.76-1.29$ (m, 56H, Cyp), 1.02-0.91 (m, 7H, Cyp), 0.41 (s, 6H (-SiMe 2$)), 0.31$ (s, 6H (-SiMe 2$))$.

${ }^{13} \mathrm{C}$ NMR $\left(\mathrm{CDCl}_{3}, 75 \mathrm{MHz}\right): \delta 136.77\left(\mathrm{C}^{*}\right), 133.86,129.52,127.97,113.99(\mathrm{C} \equiv \mathrm{C}), 110.51(\mathrm{C} \equiv \mathrm{C}), 27.44$, $27.17,22.34,22.22,1.93,-0.78$.

${ }^{29} \mathrm{Si} \mathrm{NMR}\left(\mathrm{CDCl}_{3}, 79 \mathrm{MHz}\right): \delta$ - 17.31, - 22.50, -65.90, -66.54, -108.65

EA: Anal. calcd for $\mathrm{C}_{47} \mathrm{H}_{80} \mathrm{O}_{13} \mathrm{Si}_{10}$ (\%):C, 49.78, $\mathrm{H}, 7.11$; found: $\mathrm{C}, 49.89 ; \mathrm{H}, 7.22$.
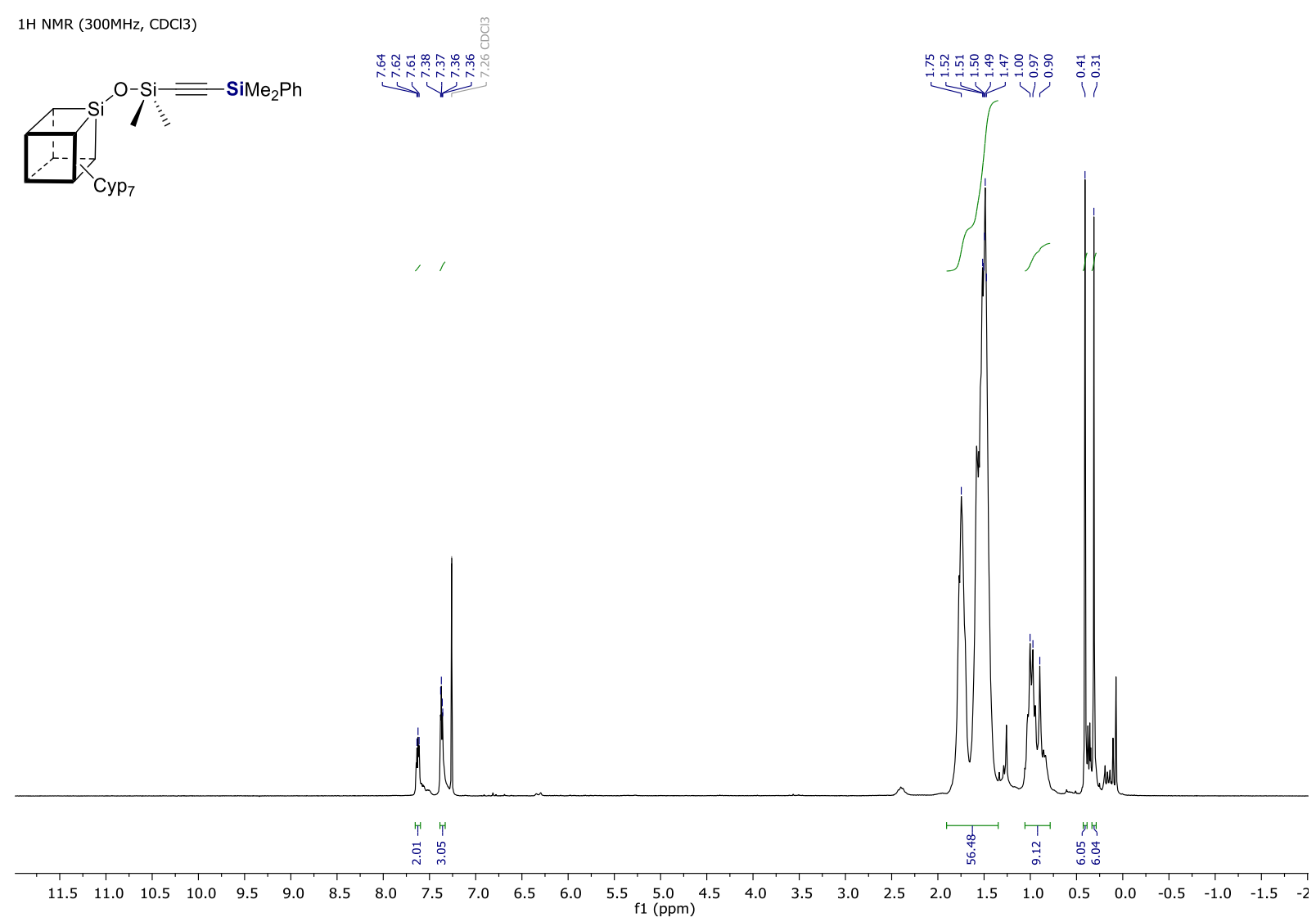

Figure S-33. ${ }^{1} \mathrm{H}$ NMR spectrum $\left(300 \mathrm{MHz}, \mathrm{CDCl}_{3}\right)$ of product $\mathrm{P}-3 \mathrm{~b}$. 


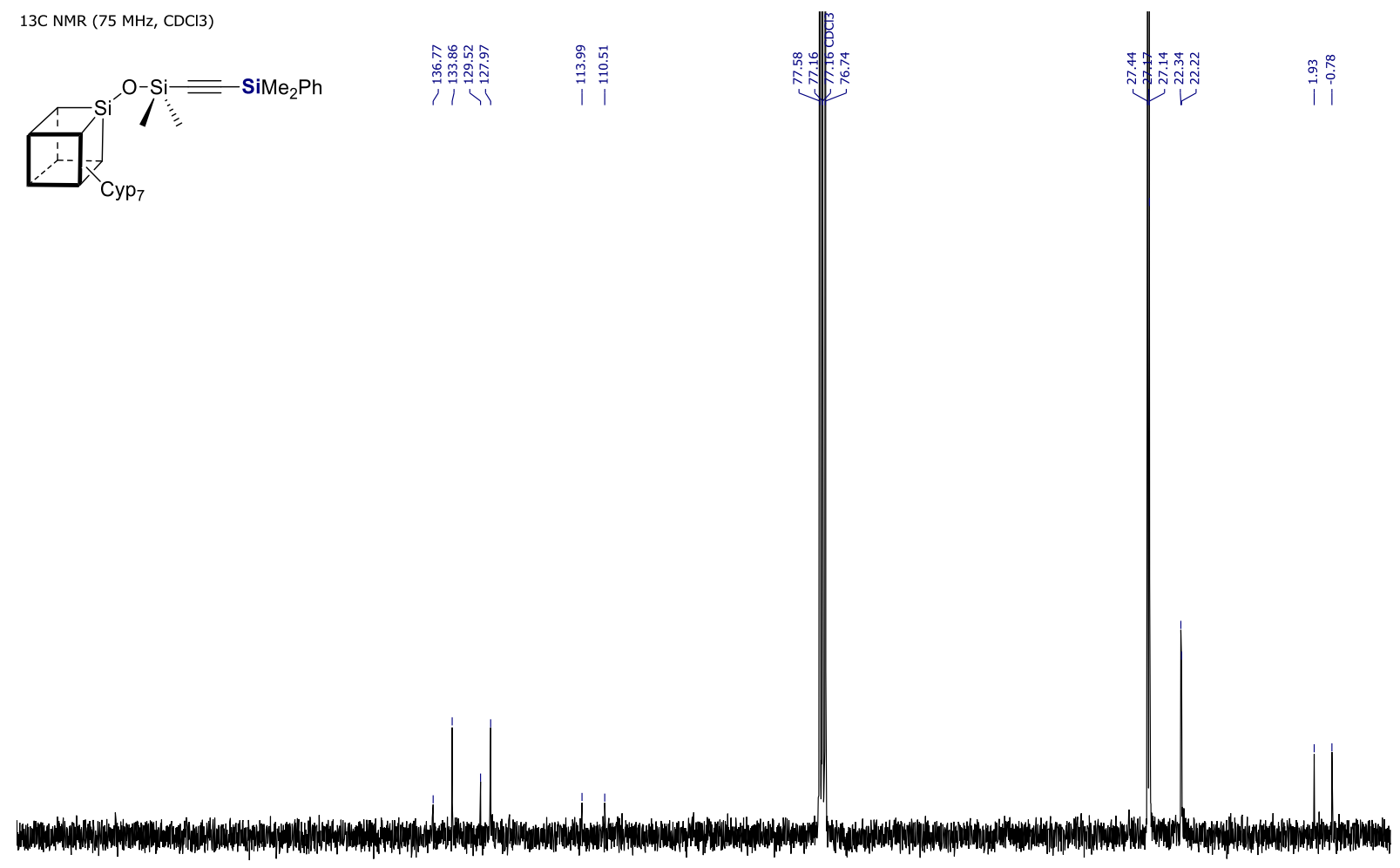

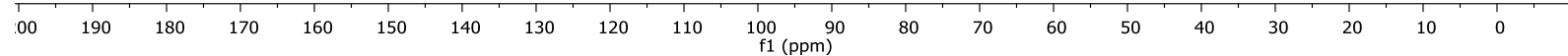

Figure S-34. ${ }^{13} \mathrm{C}$ NMR spectrum $\left(75 \mathrm{MHz}, \mathrm{CDCl}_{3}\right.$ ) of product $\mathrm{P}-3 \mathrm{~b}$
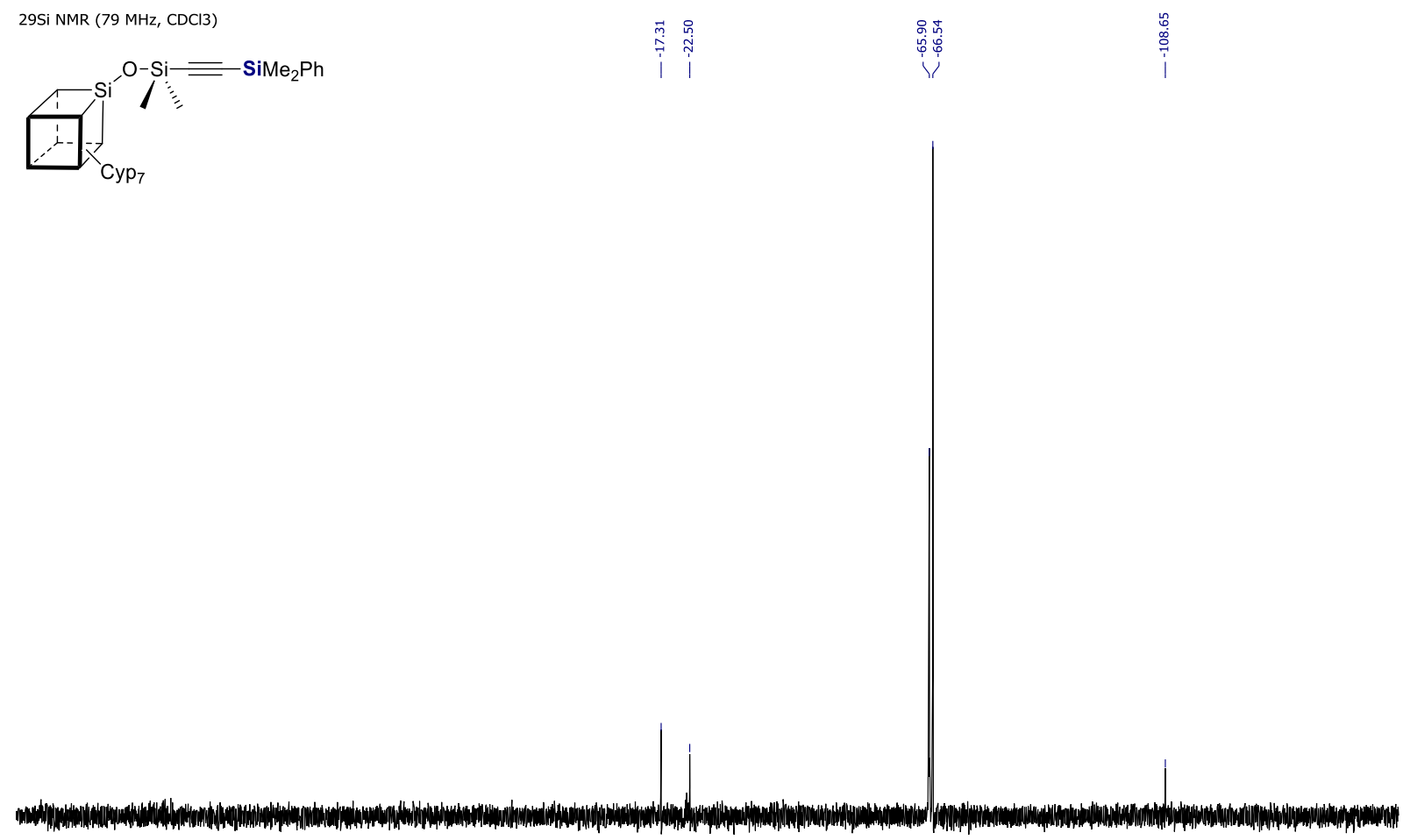

$\begin{array}{lllllllllllllllllllllllll}90 & 80 & 70 & 60 & 50 & 40 & 30 & 20 & 10 & 0 & -10 & -20 & -30 & -40 & -50 & -60 & -70 & -80 & -90 & -100 & -110 & -120 & -130 & -140 & -1 !\end{array}$

Figure S-35. ${ }^{29} \mathrm{Si} \mathrm{NMR} \mathrm{spectrum}\left(79 \mathrm{MHz}, \mathrm{CDCl}_{3}\right)$ of product $\mathrm{P}-3 \mathrm{~b}$ 
White solid. Isolated yield $85 \%$.

${ }^{1} \mathrm{H}$ NMR (CDCl, $\left.300 \mathrm{MHz}\right): \delta 1.75-1.47$ (m, 56H, Cyp), 1.01-0.89 (m, 9H, -Me + 7H, Cyp), 0.64-0.53 (m, $\left.6 \mathrm{H},\left(-\mathrm{CH}_{2}-\right)\right), 0.28\left(\mathrm{~s}, 6 \mathrm{H},-\mathrm{SiMe}_{2}\right)$.

${ }^{13} \mathrm{C}$ NMR $\left(\mathrm{CDCl}_{3}, 75 \mathrm{MHz}\right): \delta 113.41(\mathrm{C} \equiv \mathrm{C}), 110.47(\mathrm{C} \equiv \mathrm{C}), 27.43,27.17,22.32,7.53,4.33,2.03$.

${ }^{29} \mathrm{Si}$ NMR $\left(\mathrm{CDCl}_{3}, 125 \mathrm{MHz}\right): \delta-7.95,-17.95,-65.93,66.55,-108.76$.

EA: Anal. calcd for $\mathrm{C}_{45} \mathrm{H}_{84} \mathrm{O}_{13} \mathrm{Si}_{10}$ (\%):C, 48.52, $\mathrm{H}, 7.60$; found: $\mathrm{C}, 48.75 ; \mathrm{H}, 7.78$.
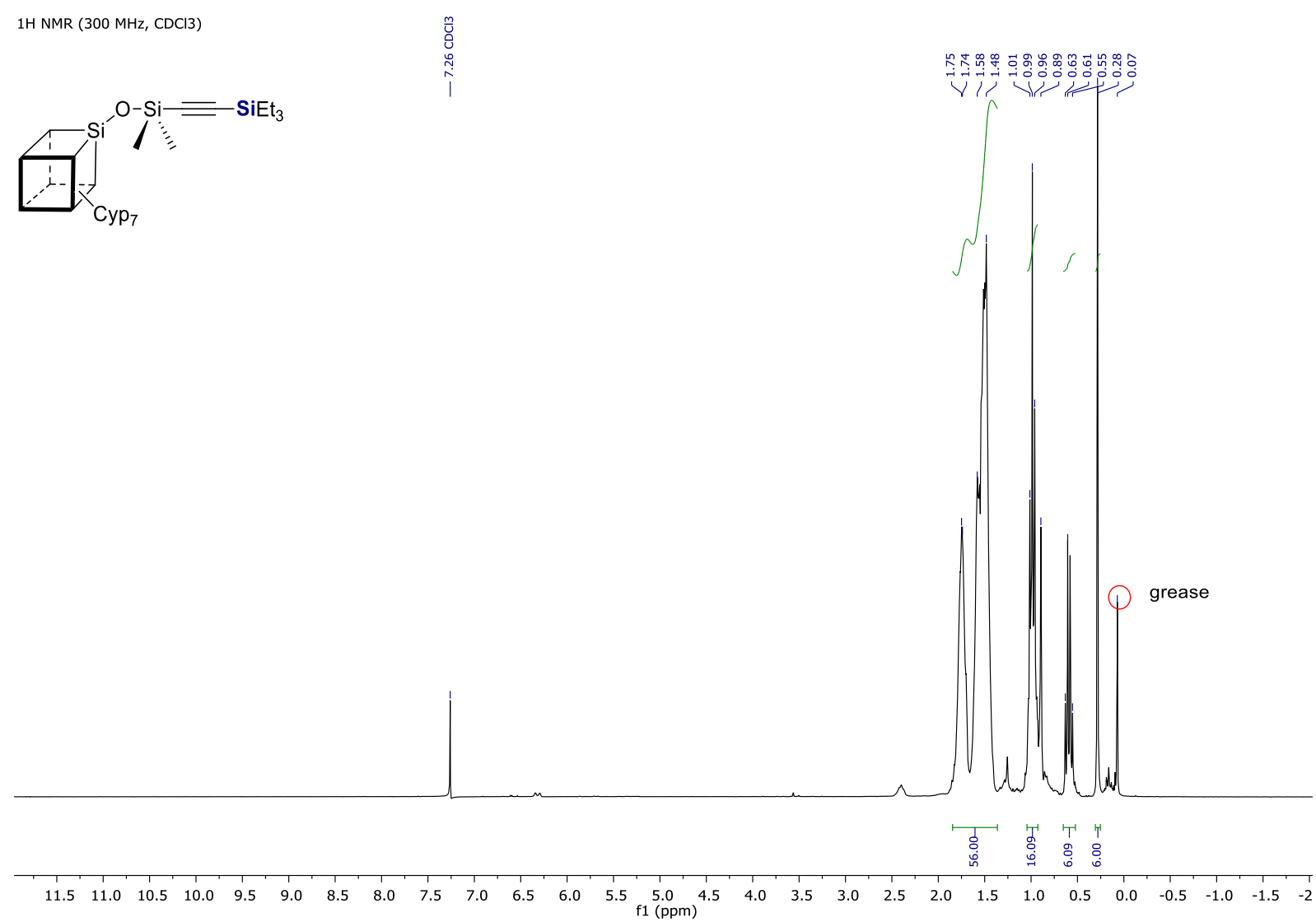

Figure S-36. ${ }^{1} \mathrm{H}$ NMR spectrum $\left(300 \mathrm{MHz}, \mathrm{CDCl}_{3}\right)$ of product $\mathrm{P}-3 \mathrm{C}$ 

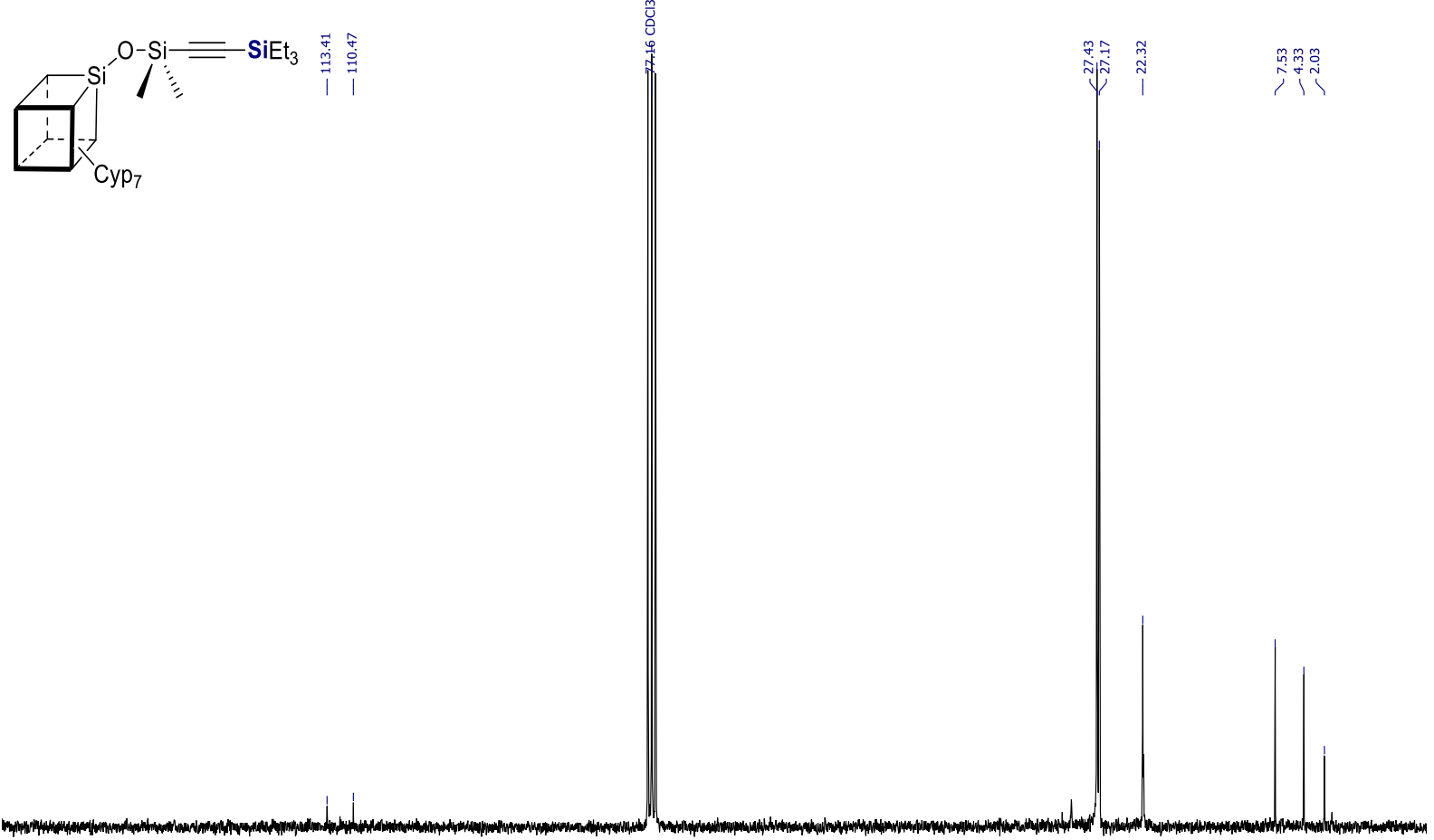

140

130

$10 \quad 100 \quad 90 \quad 80$

70
$f 1(p p m)$

$60 \quad 50$

40

$30 \quad 20 \quad 10$

Figure S-37. ${ }^{13} \mathrm{C} \mathrm{NMR}$ spectrum $\left(75 \mathrm{MHz}, \mathrm{CDCl}_{3}\right.$ ) of product $\mathrm{P}-3 \mathrm{C}$.

29Si NMR (79MHz, CDCl3)

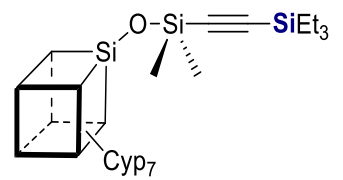

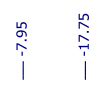
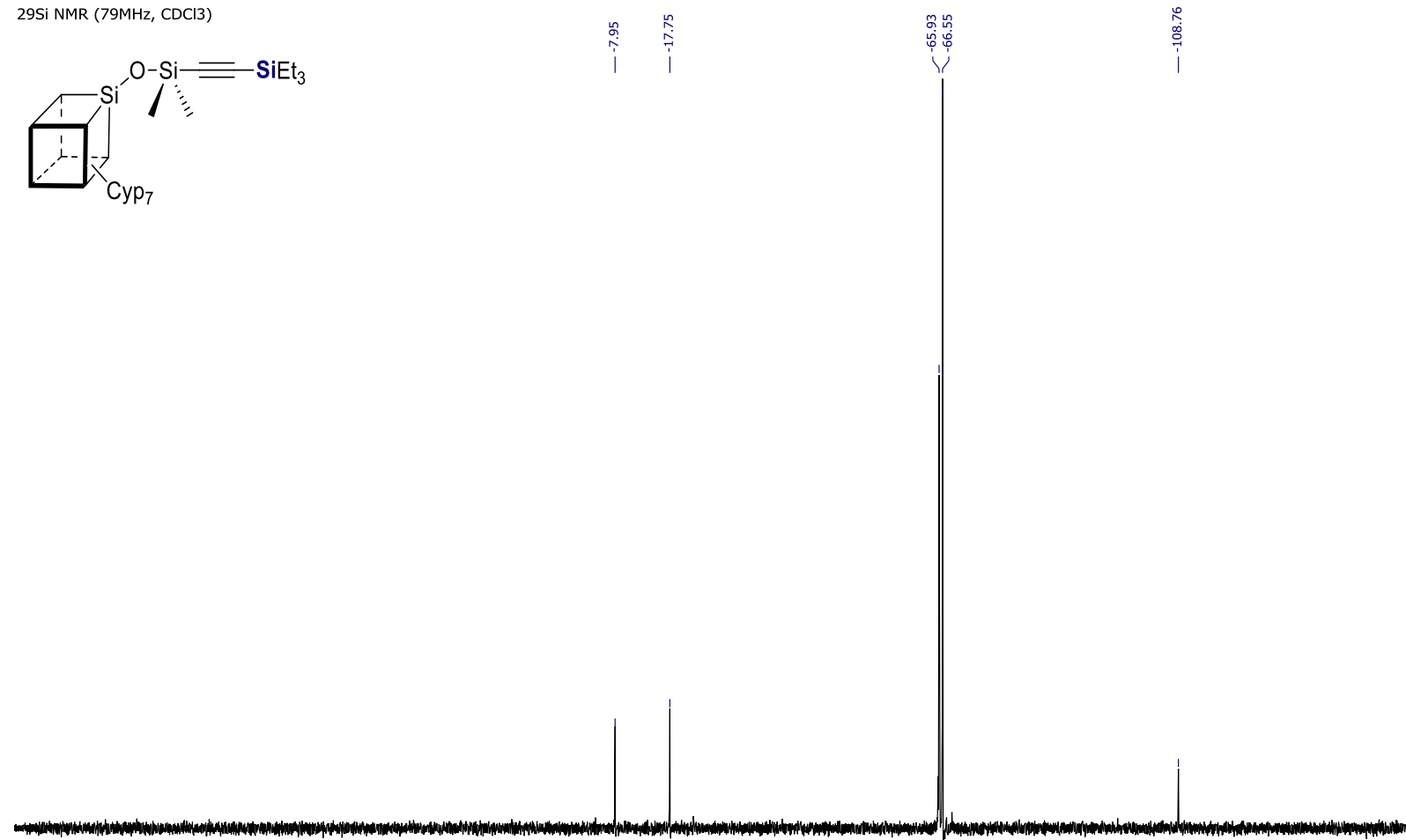

$\begin{array}{llllllllllllllll}90 & 80 & 70 & 60 & 50 & 40 & 30 & 20 & 10 & 0 & -10 & \begin{array}{c}-20 \\ \mathrm{f} 1(\mathrm{pmm})\end{array} & -40 & -50 & -60\end{array}$

Figure S-38. ${ }^{29} \mathrm{Si} \mathrm{NMR} \mathrm{spectrum}\left(79 \mathrm{MHz}, \mathrm{CDCl}_{3}\right)$ of product P-3c. 
White solid. Isolated yield $65 \%$.

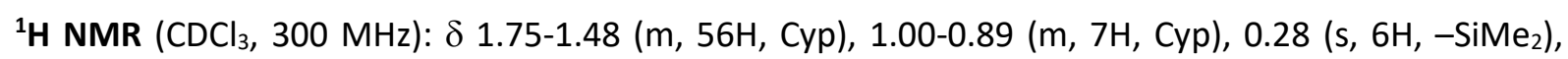
0.15 (s, 3H (-SiMe $\left.\left(\mathrm{OSiMe}_{3}\right)_{2}\right), 0.14\left(\mathrm{~s}, 18 \mathrm{H}\left(-\mathrm{SiCH}_{3}\left(\mathrm{OSiMe}_{3}\right)_{2}\right)\right.$.

${ }^{13} \mathrm{C}$ NMR $\left(\mathrm{CDCl}_{3}, 100 \mathrm{MHz}\right.$ ): $\delta 110.83$ ( $\left.\mathrm{C} \equiv \mathrm{C}\right), 108.75$ (C $\left.\equiv \mathrm{C}\right), 27.44,27.42,22.39,22.23,1.79,1.71,1.62$. ${ }^{29} \mathrm{Si} \mathrm{NMR}\left(\mathrm{CDCl}_{3}, 79 \mathrm{MHz}\right): \delta 10.13,-17.15,-53.80,-65.93,66.55,-108.73$.

EA: Anal. calcd for $\mathrm{C}_{46} \mathrm{H}_{90} \mathrm{O}_{15} \mathrm{Si}_{12}$ (\%):C, 45.28, $\mathrm{H}, 7.43$; found: $\mathrm{C}, 45.44 ; \mathrm{H}, 7.65$.
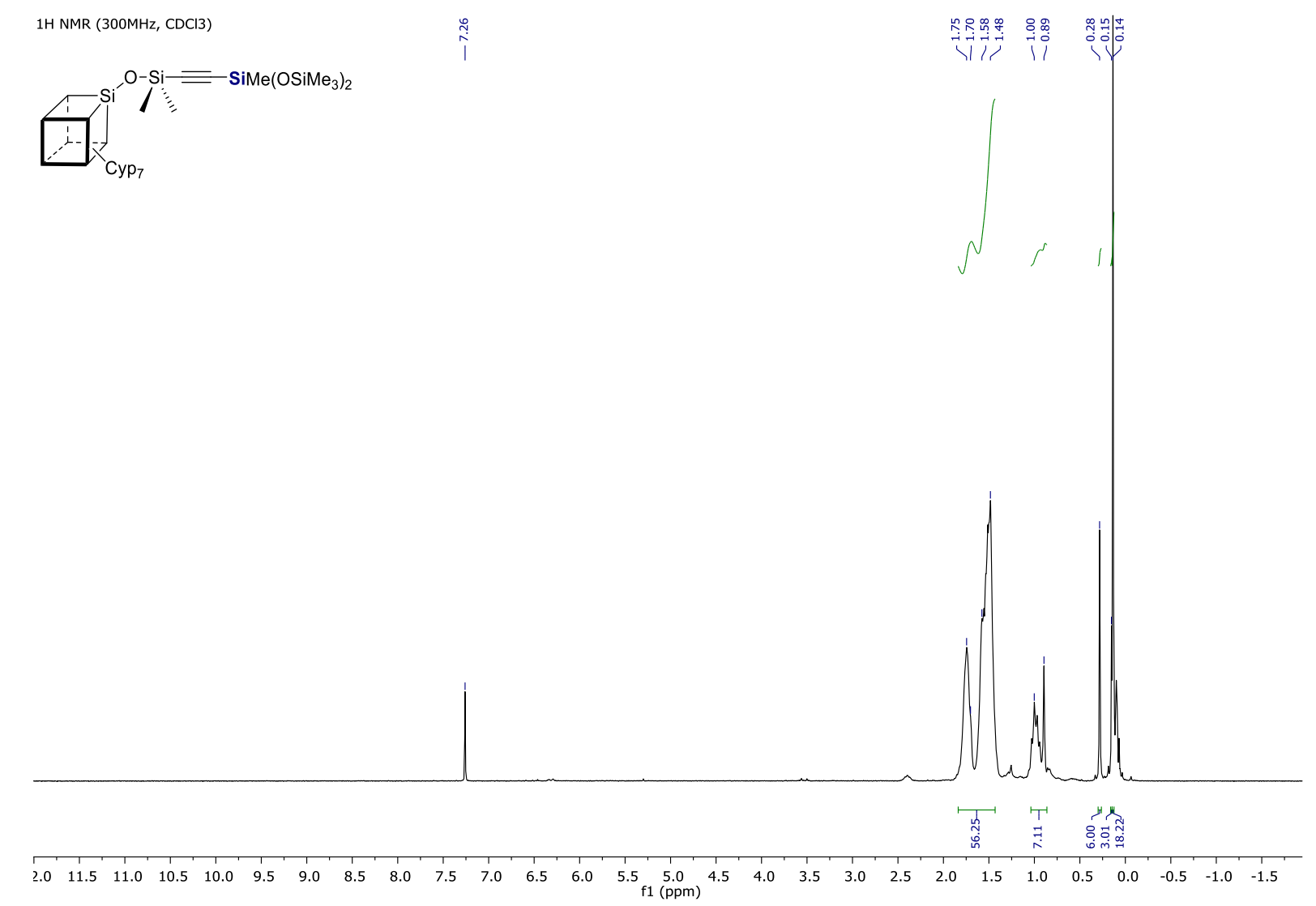

Figure S-39. ${ }^{1} \mathrm{H}$ NMR spectrum $\left(300 \mathrm{MHz}, \mathrm{CDCl}_{3}\right)$ of product $\mathrm{P}-3 \mathrm{~d}$. 
13C NMR (100MHz, CDCl3)
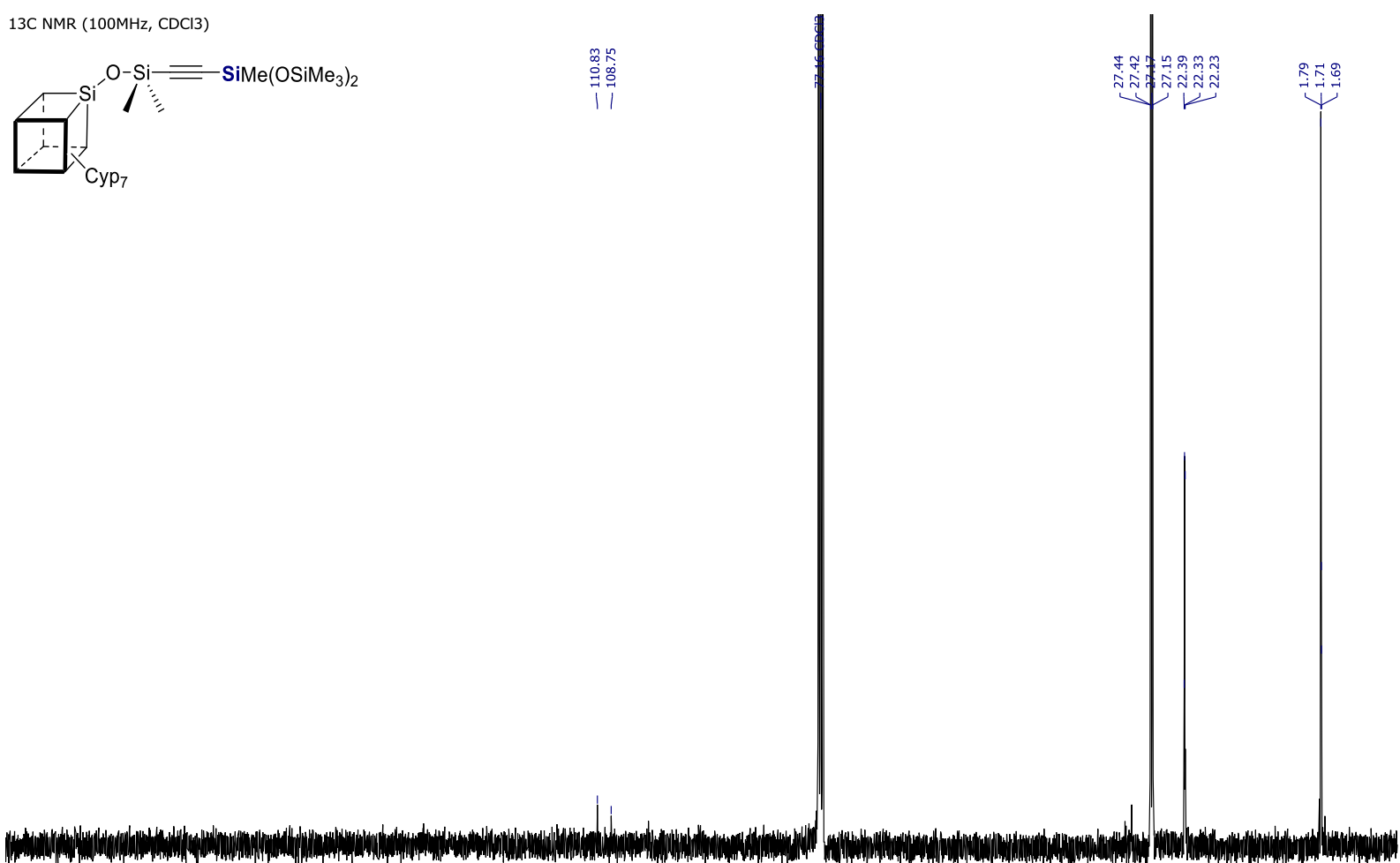

Figure S-40. ${ }^{13} \mathrm{C}$ NMR spectrum (100 MHz, CDCl ${ }_{3}$ ) of product P-3d

29Si NMR $(79 \mathrm{MHz}, \mathrm{CDCl} 3)$

SiMe $\left(\mathrm{OSiMe}_{3}\right)_{2}$

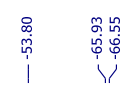

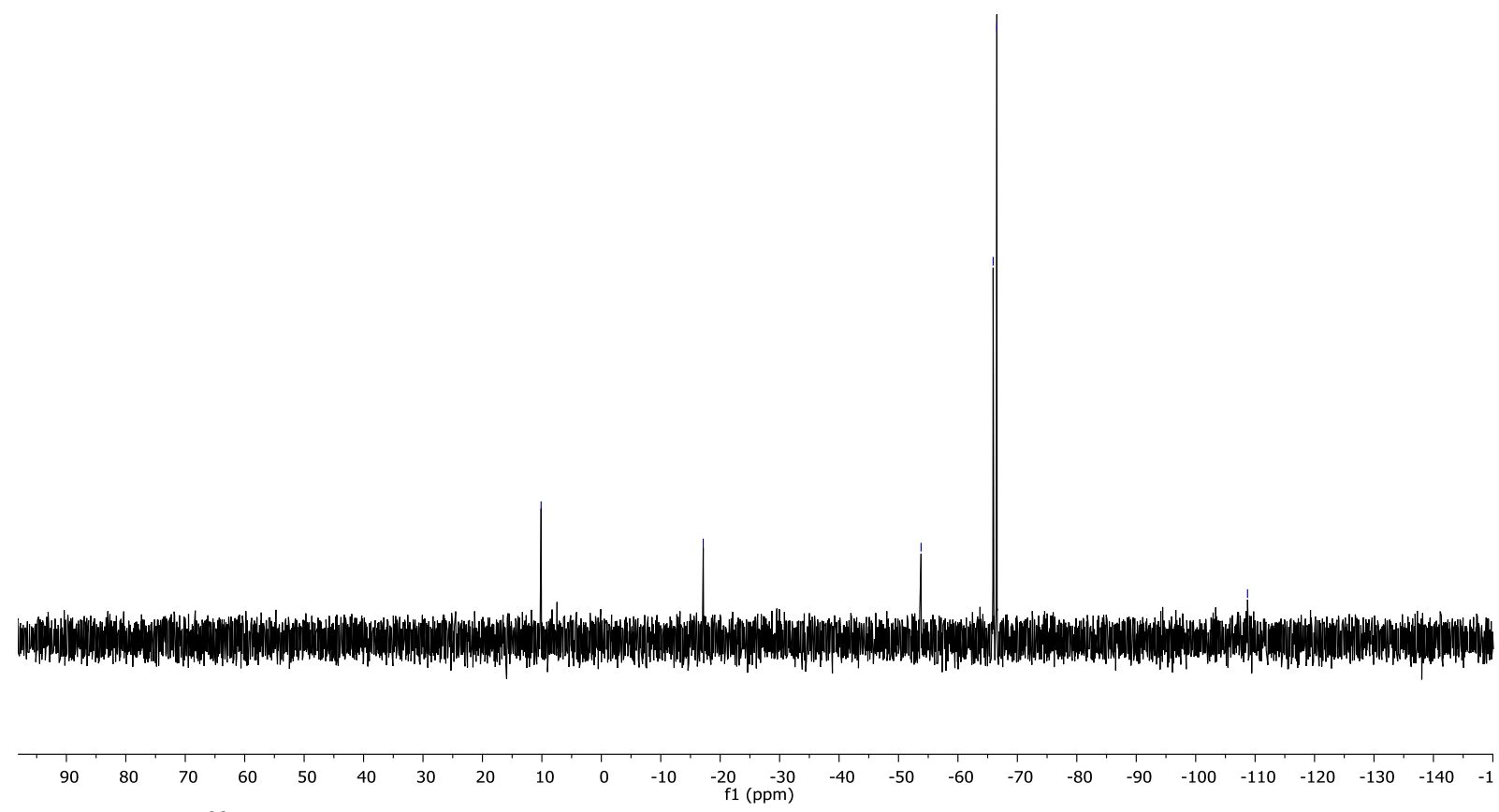

Figure S-41. ${ }^{29}$ Si NMR spectrum $\left(75 \mathrm{MHz}, \mathrm{CDCl}_{3}\right)$ of product P-3d. 
White solid. Isolated yield $80 \%$.

${ }^{1} \mathbf{H}$ NMR $\left(\mathrm{CDCl}_{3}, 300 \mathrm{MHz}\right): \delta$ 7.54-7.51 (m, 2H, Ph), 7.39-7.34 (m, 3H, Ph), 1,75-1,73 (m, 28H, Cy); 1,24-1,21 (m, 42H, Cy), 0,80-0,72 (m, 7H), 0.41 (s, 6H-SiMe 2 Ph), 0.31 (s, 6H, SiMe $)$.

${ }^{13} \mathrm{C} \mathrm{NMR}\left(\mathrm{CDCl}_{3}, 75 \mathrm{MHz}\right): \delta 136.74,133.85,129.53,127.98,113.92$ (C $\left.\equiv \mathrm{C}\right), 110.57$ (C $\left.\equiv \mathrm{C}\right), 27.64,27.57$, 27.04, 26.98, 26.77, 26.66, 23.27, 23.23, 23.10, 2.02, 1.18, -0.76.

${ }^{29} \mathrm{Si} \mathrm{NMR}\left(\mathrm{CDCl}_{3}, 79 \mathrm{MHz}\right): \delta-17.46,-22.45,-67.99,-68.66,-68.68,-108.63$.

EA: Anal. calcd for $\mathrm{C}_{54} \mathrm{H}_{94} \mathrm{O}_{13} \mathrm{Si}_{10}(\%): \mathrm{C}, 52.64, \mathrm{H}, 7.69$; found: C, 52.98; $\mathrm{H}, 7.99$.

$1 \mathrm{H} \mathrm{NMR} \mathrm{(300MHz,} \mathrm{CDCl3)}$
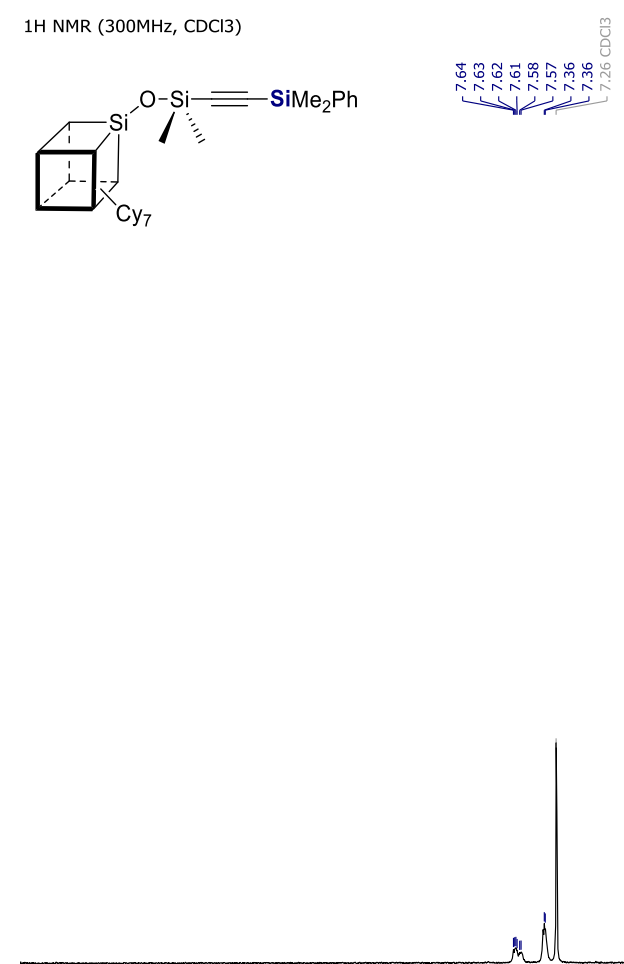

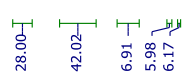

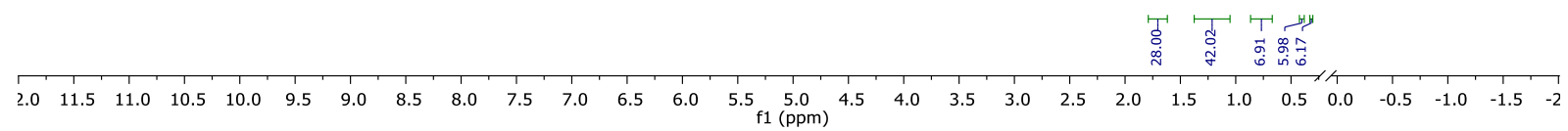

Figure S-42. ${ }^{1} \mathrm{H}$ NMR spectrum $\left(300 \mathrm{MHz}, \mathrm{CDCl}_{3}\right)$ of product $\mathrm{P}-4 \mathrm{~b}$. 


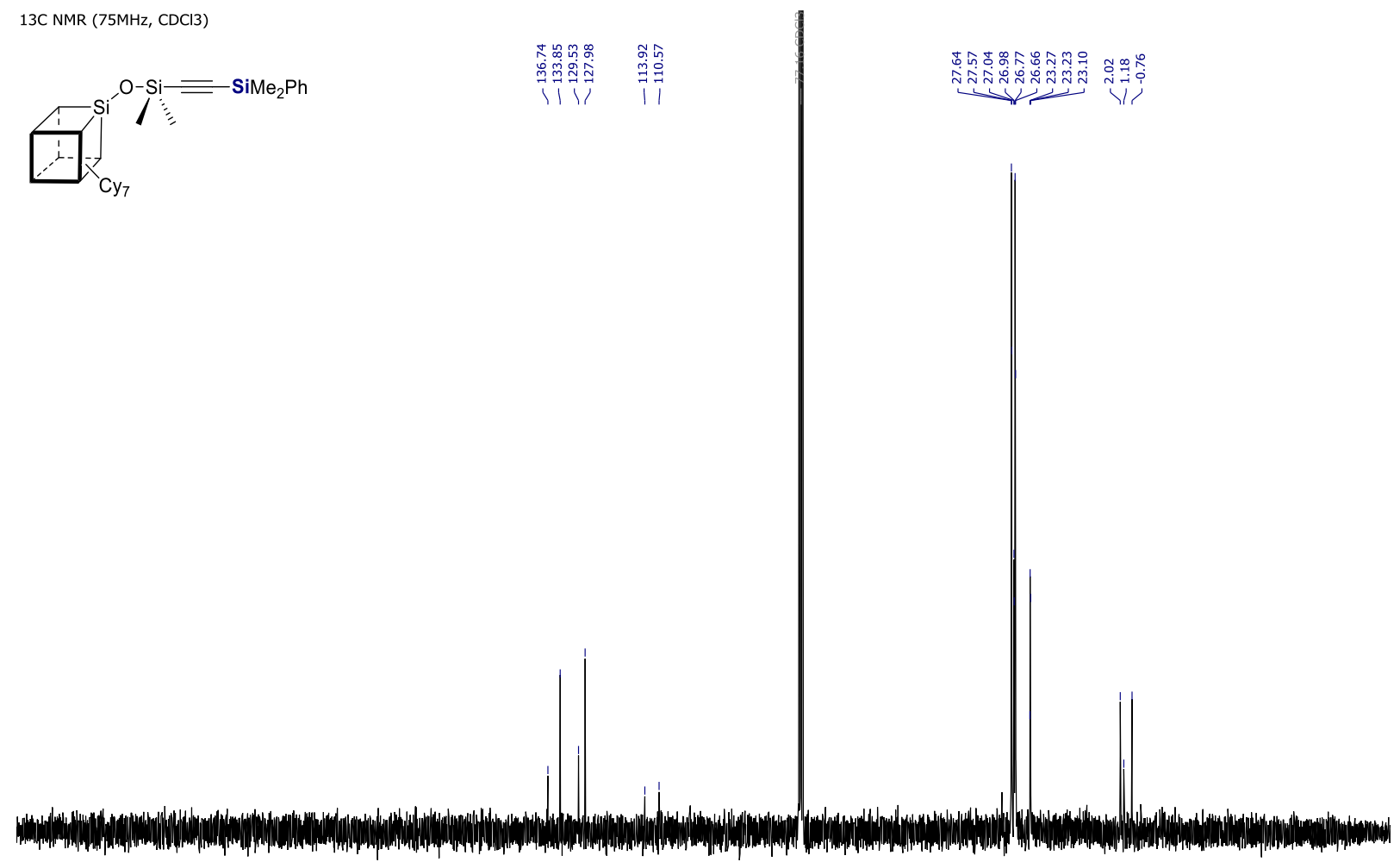

$\begin{array}{llllllllllllllllllllllllllllllllllllllllll}260 & 250 & 240 & 230 & 220 & 210 & 200 & 190 & 180 & 170 & 160 & 150 & 140 & 130 & 120 & 110 & 100 & 90 & 80 & 70 & 60 & 50 & 40 & 30 & 20 & 10 & 0 & -10 & -20 & -30 & -40 & -50 & -6\end{array}$

Figure S-43. ${ }^{13} \mathrm{C}$ NMR spectrum $\left(75 \mathrm{MHz}, \mathrm{CDCl}_{3}\right)$ of product $\mathrm{P}-4 \mathrm{~b}$.
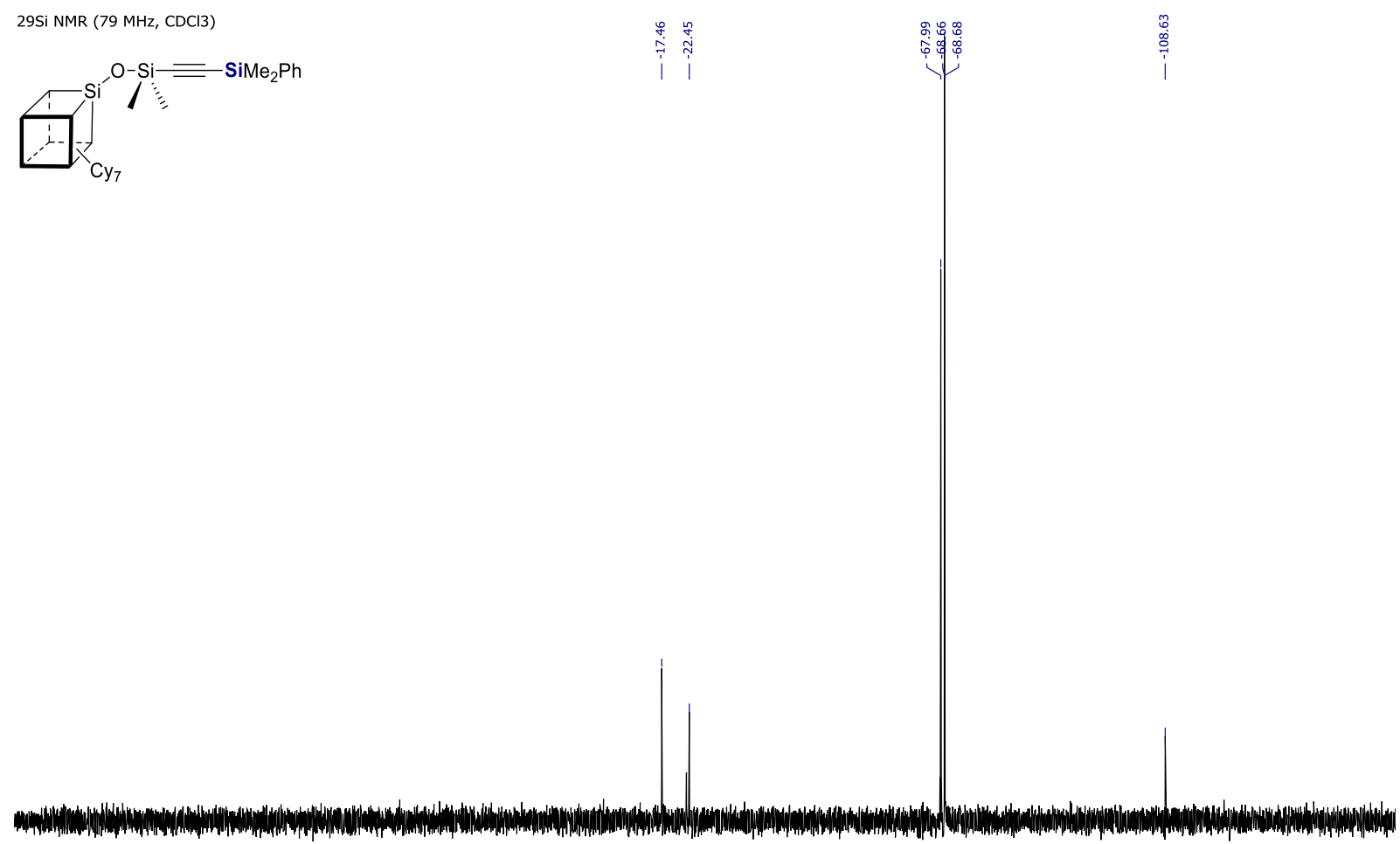

$\begin{array}{llllllllllllllllllllllllllll}90 & 80 & 70 & 60 & 50 & 40 & 30 & 20 & 10 & 0 & -10 & -20 & -30 & -40 & -50 & -60 & -70 & -80 & -90 & -100 & -110 & -120 & -130 & -140 & -1\end{array}$

Figure S-44. ${ }^{29} \mathrm{Si} \mathrm{NMR} \mathrm{spectrum}\left(79 \mathrm{MHz}, \mathrm{CDCl}_{3}\right)$ of product P-4b. 
Product obtained as white solid. Isolated yield $85 \%$.

${ }^{1} \mathrm{H}$ NMR $\left(\mathrm{CDCl}_{3}, 300 \mathrm{MHz}\right): \delta$ 1.78-1.67 (m, 28H, Cy), 1.28-1.18 (m, 42H, Cy), 0.99-0.97 (m, 9H, -Me), $0.76(\mathrm{~m}, 7 \mathrm{H}, \mathrm{Cy}), 0.64-0.56\left(\mathrm{~m}, 6 \mathrm{H},-\mathrm{CH}_{2}-\right), 0.30\left(\mathrm{~s}, 6 \mathrm{H},-\mathrm{SiMe}_{2}\right)$.

${ }^{13} \mathrm{C}$ NMR $\left(\mathrm{CDCl}_{3}, 75 \mathrm{MHz}\right): \delta 113.37(\mathrm{C} \equiv \mathrm{C}), 110.55(\mathrm{C} \equiv \mathrm{C}), 27.64,27.60,26.77,26.66,23.24,23.12$, 7.55, 4.34, 2.12 .

${ }^{29} \mathrm{Si}$ NMR $\left(\mathrm{CDCl}_{3}, 125 \mathrm{MHz}\right): \delta-7.91,-17.92,-68.04,-68.17,68.68,-108.77$

EA: Anal. calcd for $\mathrm{C}_{52} \mathrm{H}_{98} \mathrm{O}_{13} \mathrm{Si}_{10}(\%): \mathrm{C}, 51.52, \mathrm{H}, 8.15$; found: $\mathrm{C}, 51.88 ; \mathrm{H}, 8.48$.

$1 \mathrm{H} \mathrm{NMR} \mathrm{(300MHz,} \mathrm{CDCl3)}$

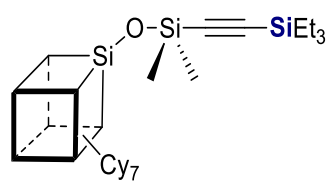

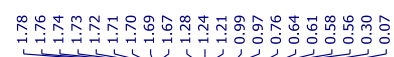
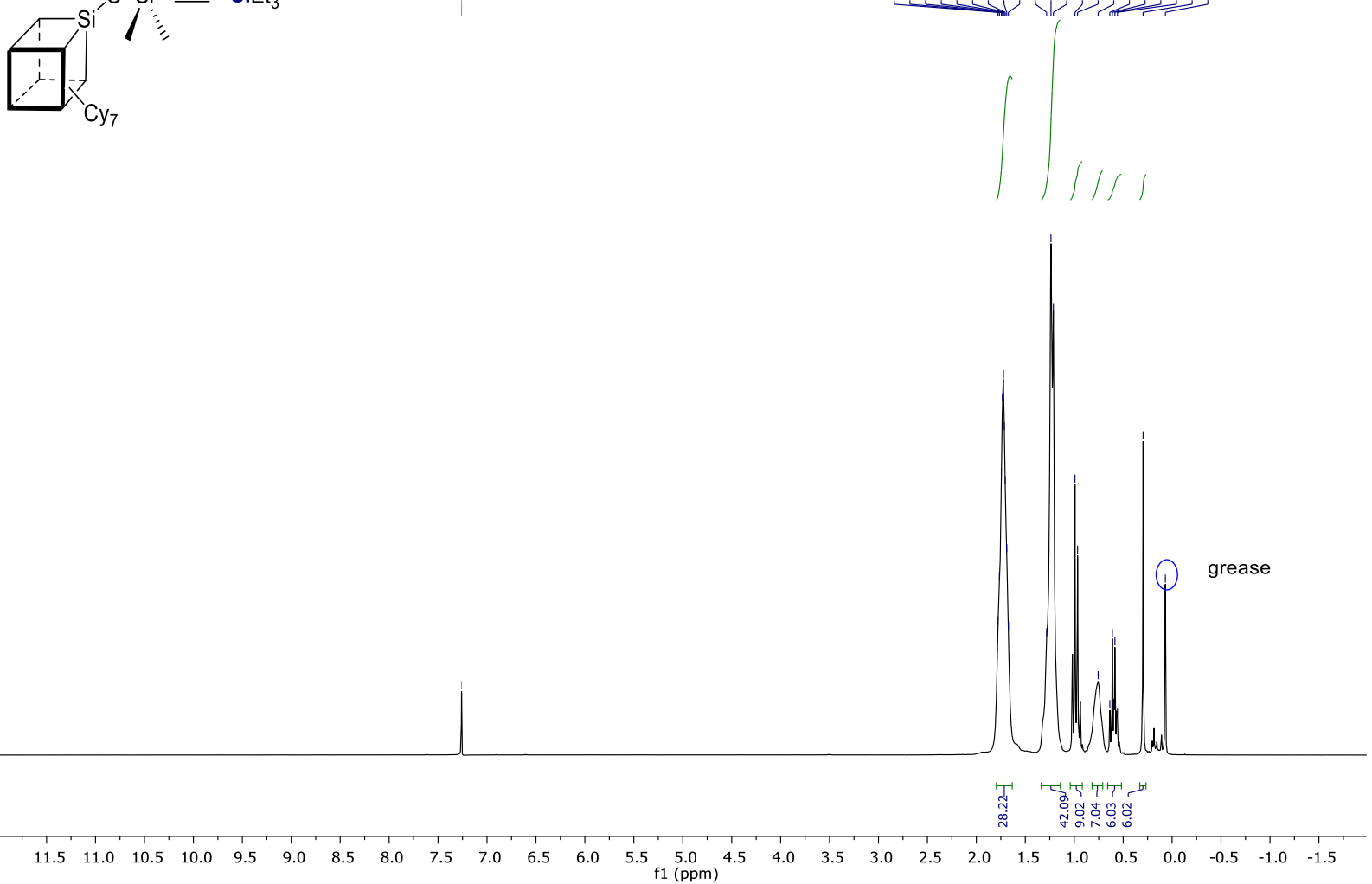

Figure S-45. ${ }^{1} \mathrm{H}$ NMR spectrum $\left(300 \mathrm{MHz}, \mathrm{CDCl}_{3}\right)$ of product $\mathrm{P}-4 \mathrm{c}$. 
$13 \mathrm{C} N \mathrm{NMR}(75 \mathrm{MHz}, \mathrm{CDCl} 3)$
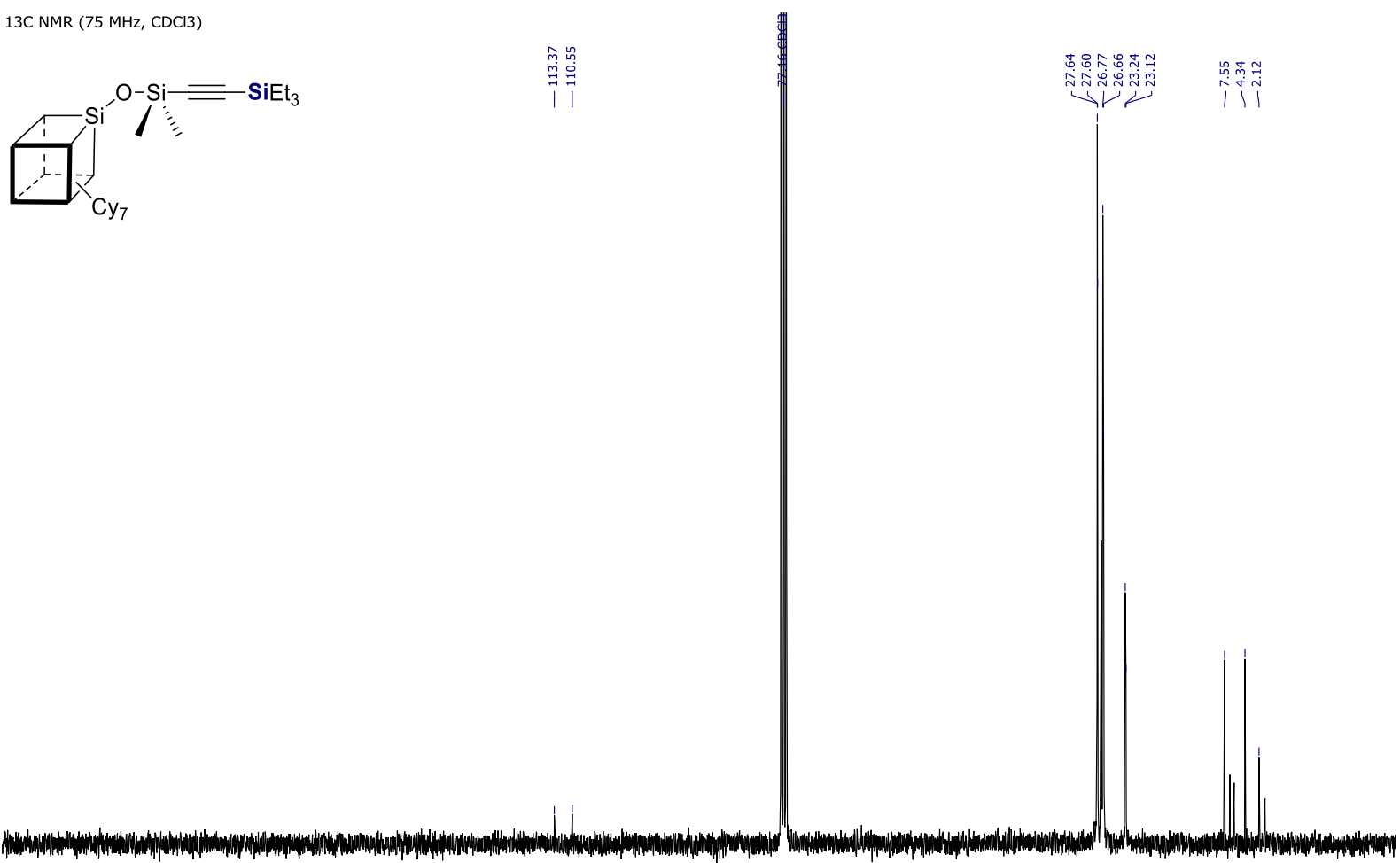

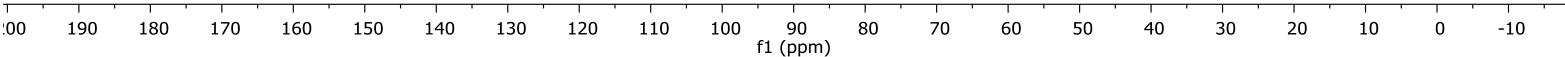

Figure S-46. ${ }^{13} \mathrm{C}$ NMR spectrum $\left(75 \mathrm{MHz}, \mathrm{CDCl}_{3}\right)$ of product P-4c.

29Si NMR $(79 \mathrm{MHz}, \mathrm{CDCl} 3)$

$\mathrm{SiEt}_{3}$

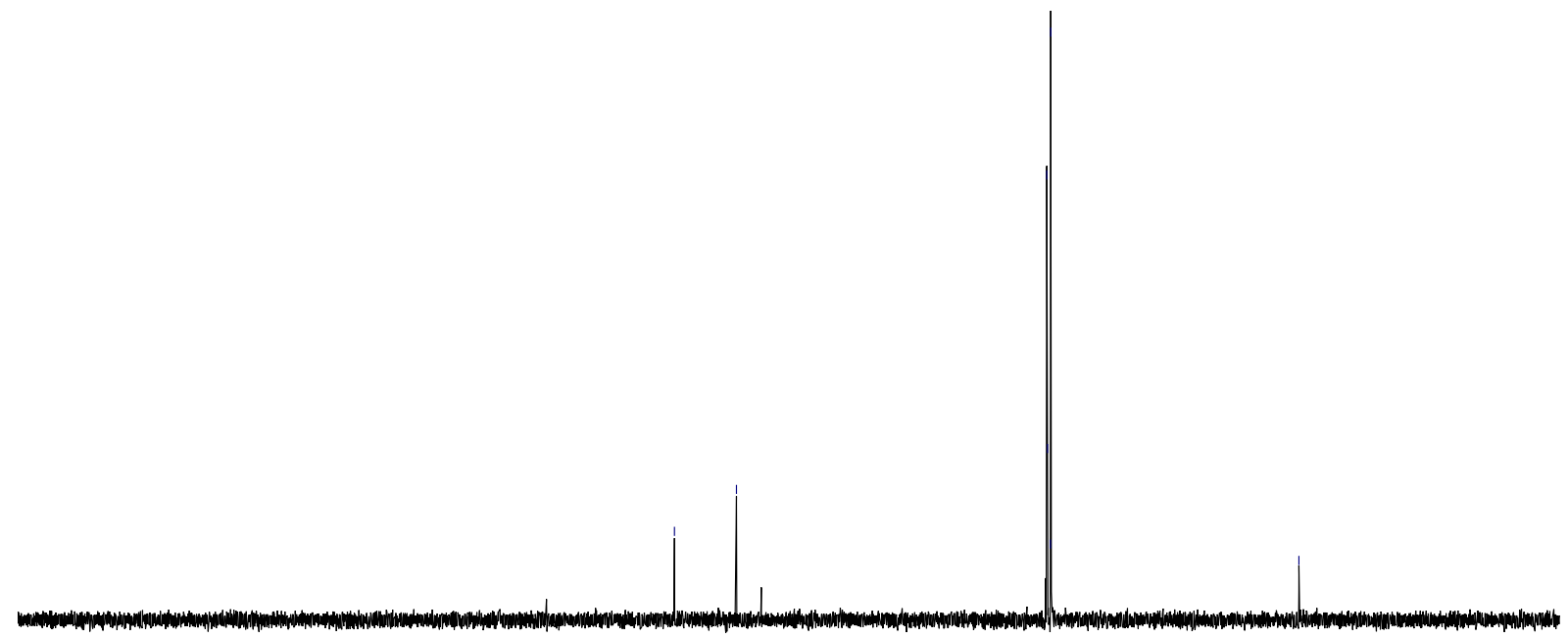

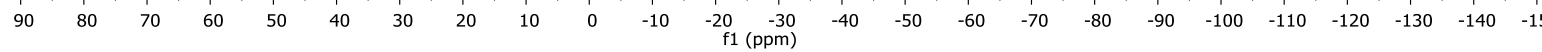

Figure S-47. ${ }^{29} \mathrm{Si} N \mathrm{NR}$ spectrum $\left(79 \mathrm{MHz}, \mathrm{CDCl}_{3}\right)$ of product $\mathrm{P}-4 \mathrm{c}$. 


\section{P-5a}

White solid. Isolated yield $82 \%$.

${ }^{1} \mathrm{H}$ NMR $\left(\mathrm{CDCl}_{3}, 400 \mathrm{MHz}\right): \delta$ 1.89-1.84 (m, 7H, iBu (-CH-)), 0.98-0.94 (m, 42H, iBu $\left.-\left(\mathrm{CH}_{3}\right)_{2}\right), 0.66-0.58$ $\left(\mathrm{m}, 14 \mathrm{H}, \mathrm{iBu}\left(-\mathrm{CH}_{2}-\right)\right), 0.19\left(\mathrm{~s}, 9 \mathrm{H},-\mathrm{SiMe}_{3}\right)$.

${ }^{13} \mathrm{C} \mathrm{NMR}\left(\mathrm{CDCl}_{3}, 125 \mathrm{MHz}\right): \delta 111.71(\mathrm{C} \equiv \mathrm{C}), 104.89(\mathrm{C} \equiv \mathrm{C}), 25.85,25.81,23.98,22.61,-0.38$.

${ }^{29} \mathrm{Si}$ NMR $\left(\mathrm{CDCl}_{3}, 99 \mathrm{MHz}\right): \delta-17.39,-67.34,-67.86,-100.57$.

EA: Anal. calcd for $\mathrm{C}_{33} \mathrm{H}_{72} \mathrm{O}_{12} \mathrm{Si}_{8}$ (\%):C,43.38, $\mathrm{H}, 7.94$; found: $\mathrm{C}, 43.62 ; \mathrm{H}, 8.25$.
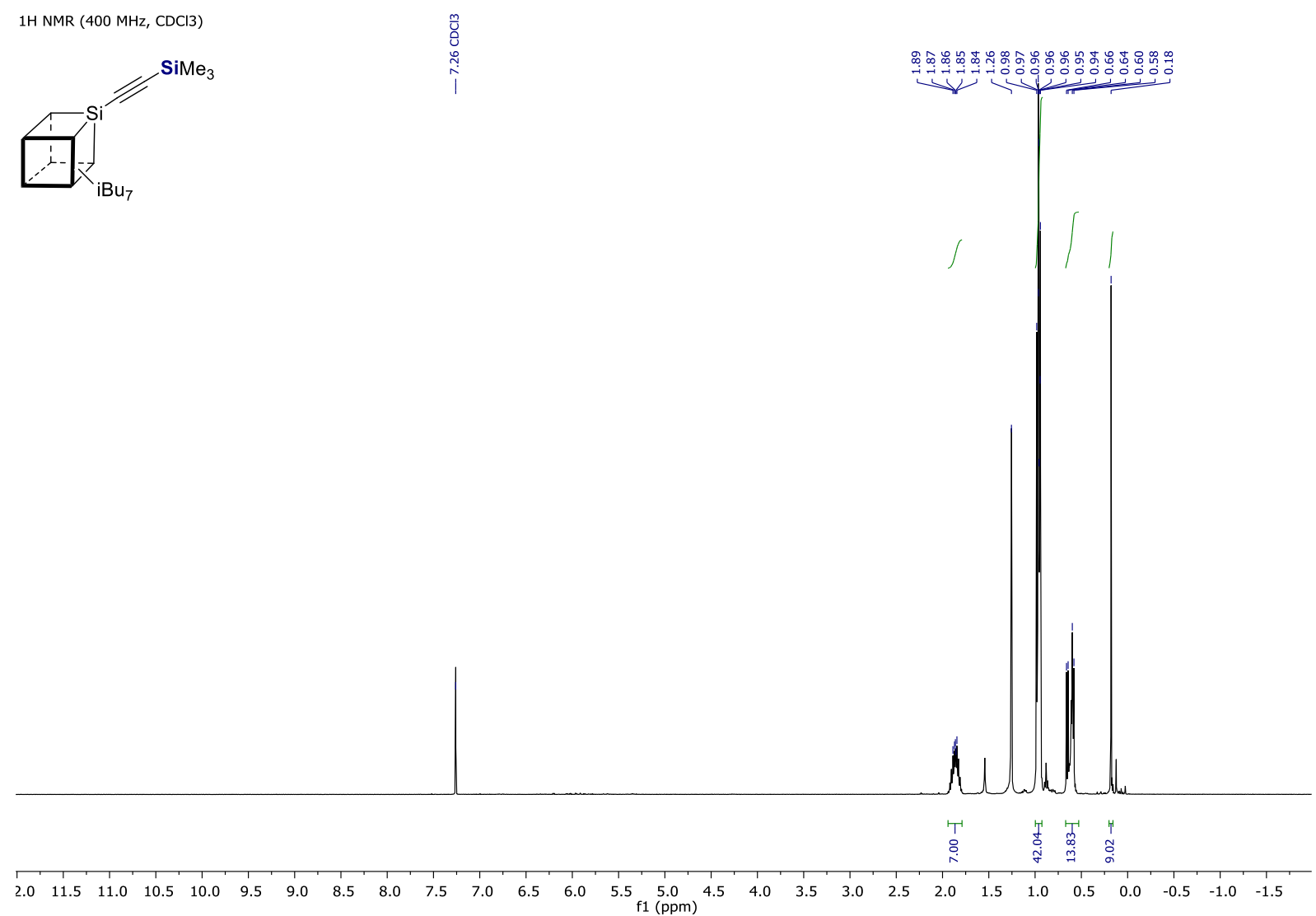

Figure S-48. ${ }^{1} \mathrm{H}$ NMR spectrum $\left(400 \mathrm{MHz}, \mathrm{CDCl}_{3}\right)$ of product $\mathrm{P}-5 \mathrm{a}$. 


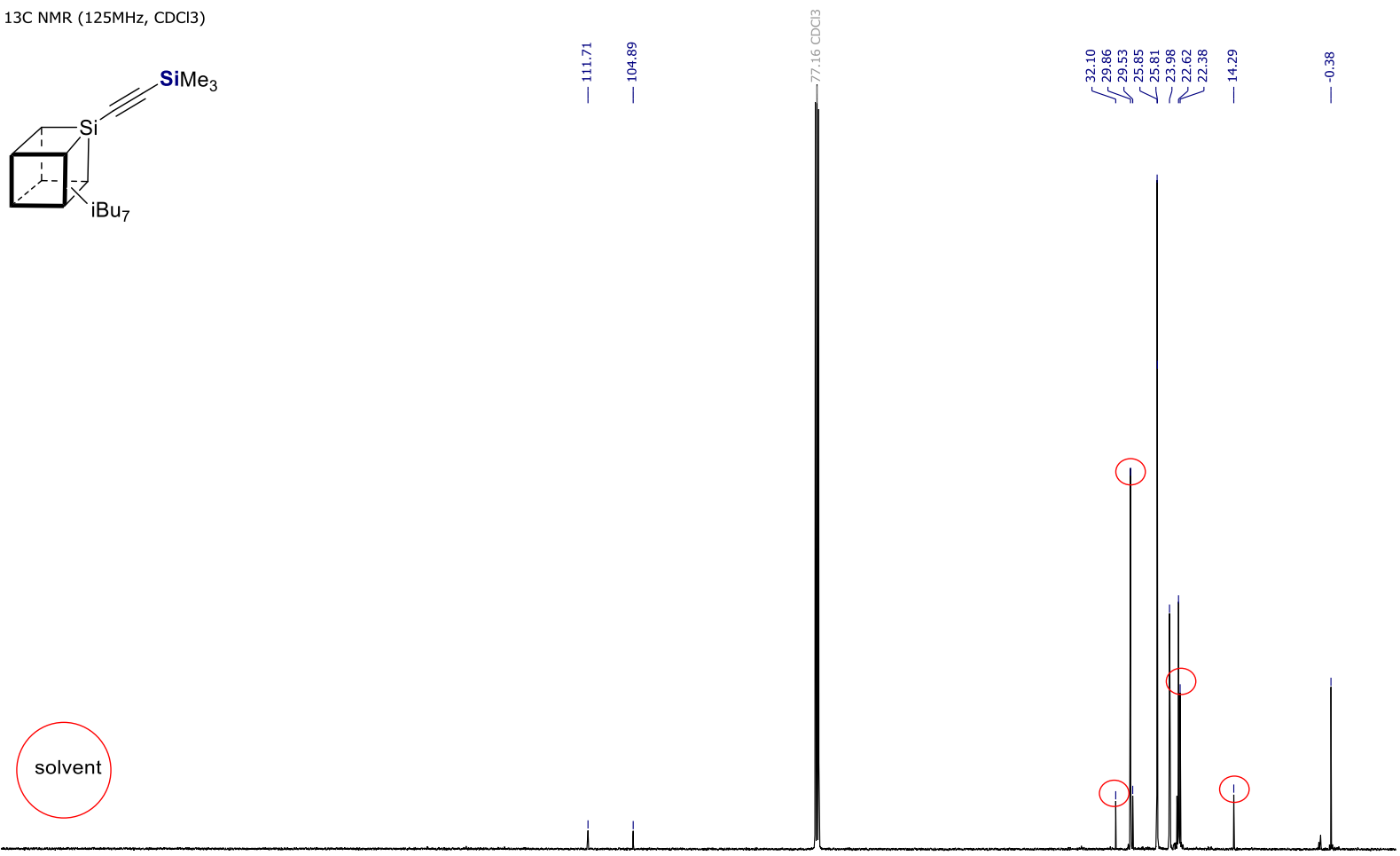

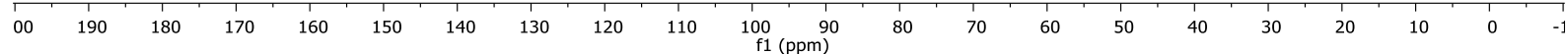

Figure S-49. ${ }^{13} \mathrm{C}$ NMR spectrum $\left(125 \mathrm{MHz}, \mathrm{CDCl}_{3}\right)$ of product $\mathrm{P}-5 \mathrm{a}$.

$29 \mathrm{Si} \mathrm{NMR} \mathrm{(99} \mathrm{MHz,} \mathrm{CDCl3)}$
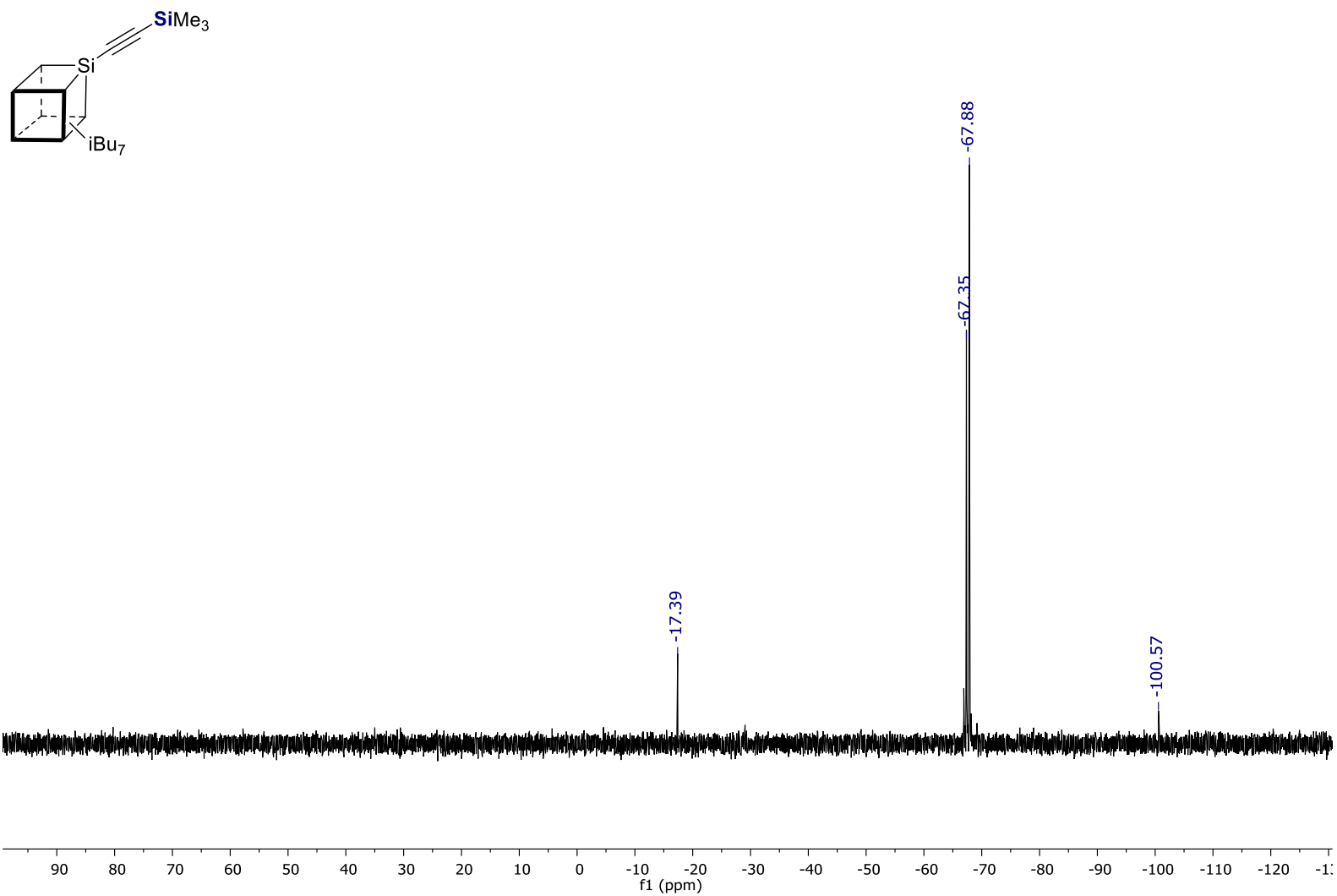

Figure S-50. ${ }^{29} \mathrm{Si} \mathrm{NMR} \mathrm{spectrum} \mathrm{(99} \mathrm{MHz}_{2} \mathrm{CDCl}_{3}$ ) of product $\mathrm{P}-5 \mathrm{a}$. 


\section{$\mathbf{P}-5 \mathrm{C}$}

White solid. Isolated yield $84 \%$.

${ }^{1} \mathrm{H}$ NMR $\left(\mathrm{CDCl}_{3}, 400 \mathrm{MHz}\right): \delta$ 1.89-1.80 (m, 7H, iBu (-CH-)), 0.99-0.94 (m, 42H, iBu +9H, Et), 0.61-0.59 (m, 14H, iBu + 6H, Et).

${ }^{13} \mathrm{C}$ NMR $\left(\mathrm{CDCl}_{3}, 75 \mathrm{MHz}\right): \delta 109.21(\mathrm{C} \equiv \mathrm{C}), 106.22(\mathrm{C} \equiv \mathrm{C}), 25.85,25.80,24.01,22.62,7.41,4.16$.

${ }^{29} \mathrm{Si}$ NMR $\left(\mathrm{CDCl}_{3}, 99 \mathrm{MHz}\right): \delta-0.80(\mathrm{HP}),-6.79,-66.73,-67.38,-67.89,-100.71$.

EA: Anal. calcd for $\mathrm{C}_{36} \mathrm{H}_{78} \mathrm{O} 12 \mathrm{Si9}$ (\%):C, 45.24, $\mathrm{H}, 8.23$; found: $\mathrm{C}, 45.39 ; \mathrm{H}, 8.73$.

$1 \mathrm{H} \mathrm{NMR} \mathrm{(300MHz,} \mathrm{CDCl3)}$
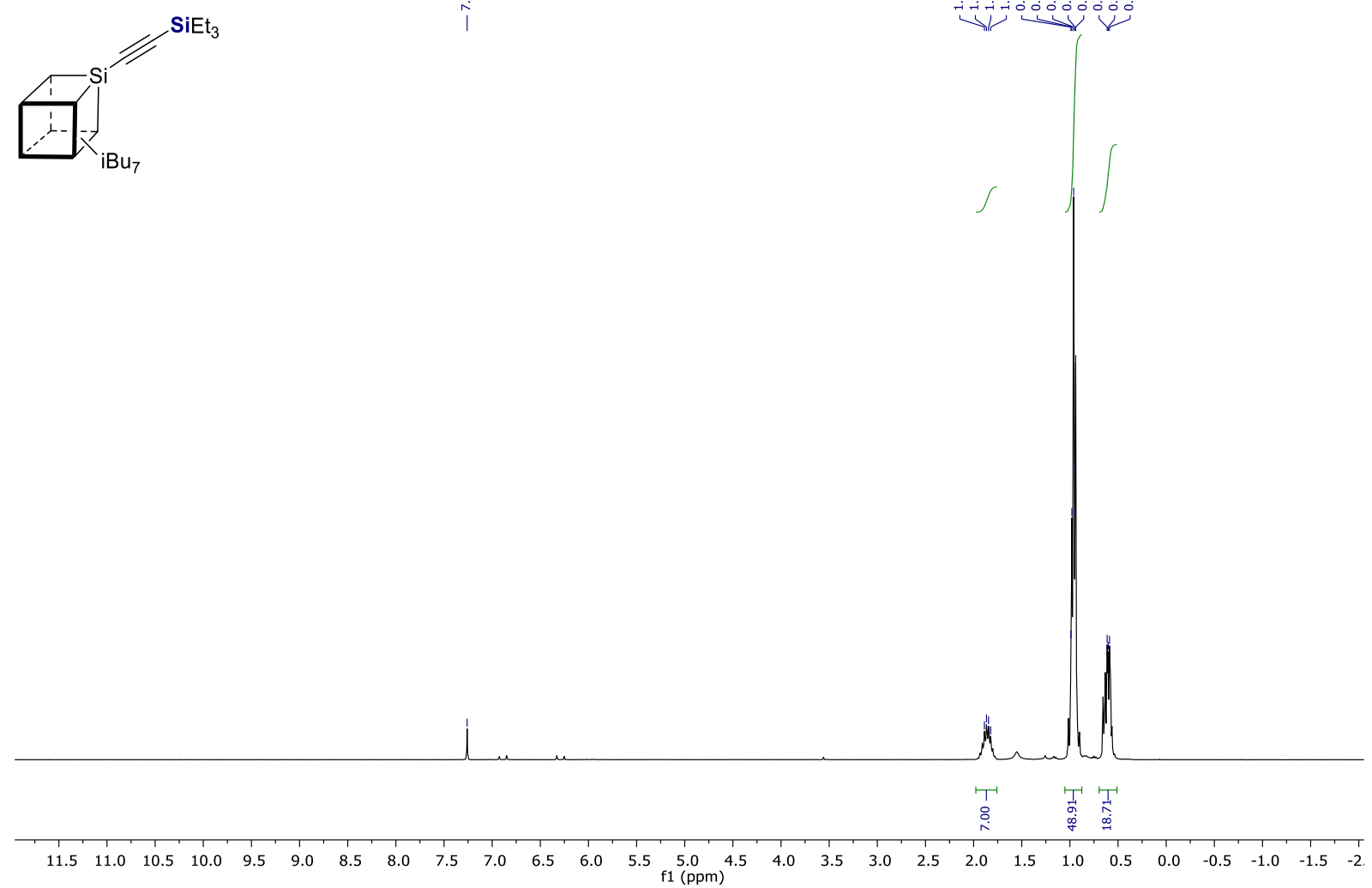

Figure S-51. ${ }^{1} \mathrm{H}$ NMR spectrum $\left(300 \mathrm{MHz}, \mathrm{CDCl}_{3}\right)$ of product $\mathrm{P}-5 \mathrm{C}$. 

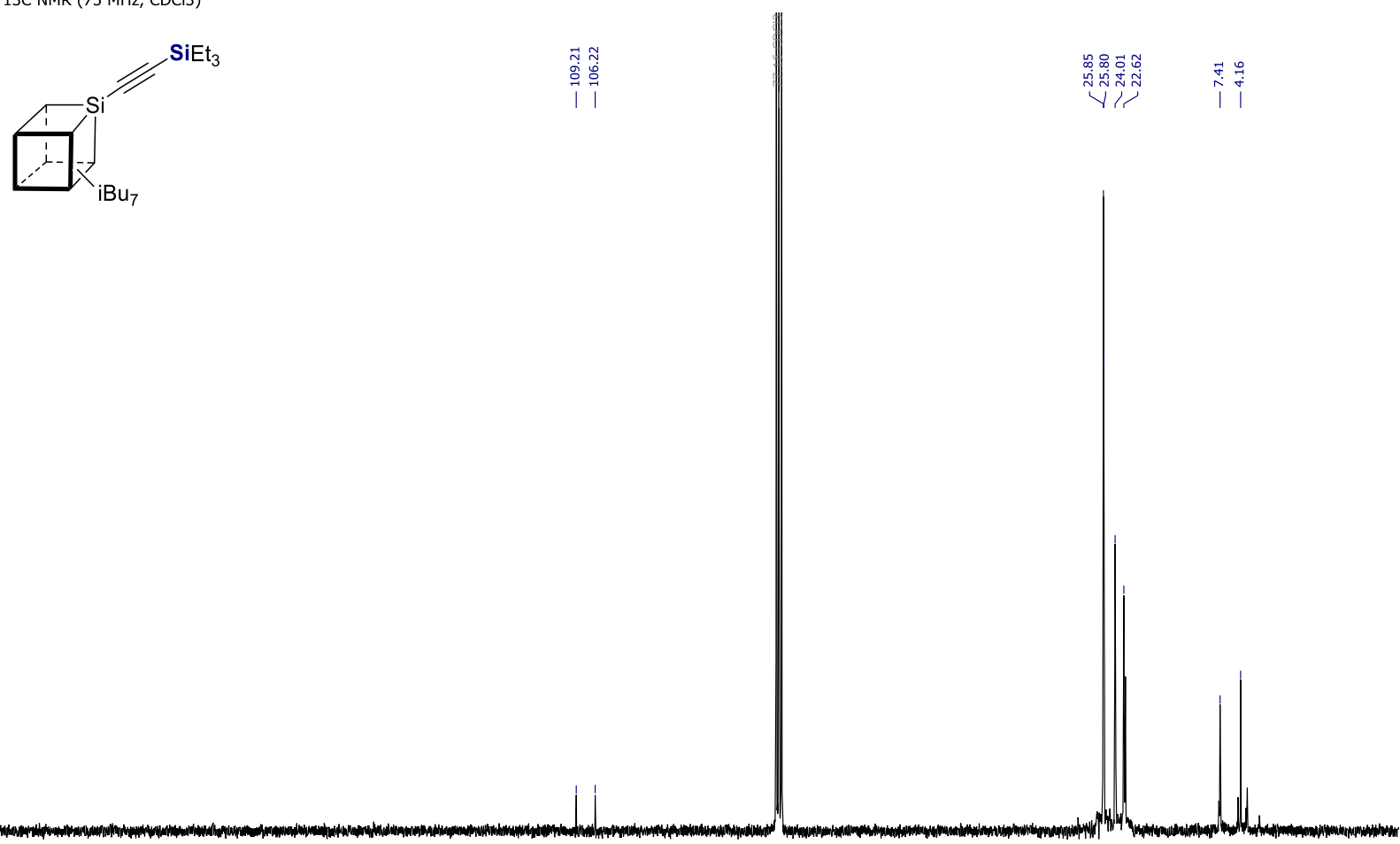

\begin{tabular}{lllllllllllllllllllllll}
\hline 00 & 190 & 180 & 170 & 160 & 150 & 140 & 130 & 120 & 110 & 100 & $\underset{\mathrm{f} 1}{90}(\mathrm{ppm})$ & 80 & 70 & 60 & 50 & 40 & 30 & 20 & 10 & 0 & -10 & -2
\end{tabular}

Figure S-52. ${ }^{13} \mathrm{C}$ NMR spectrum $\left(75 \mathrm{MHz}, \mathrm{CDCl}_{3}\right)$ of product $\mathrm{P}-5 \mathrm{c}$.

29Si NMR (79MHz, CDCl3)
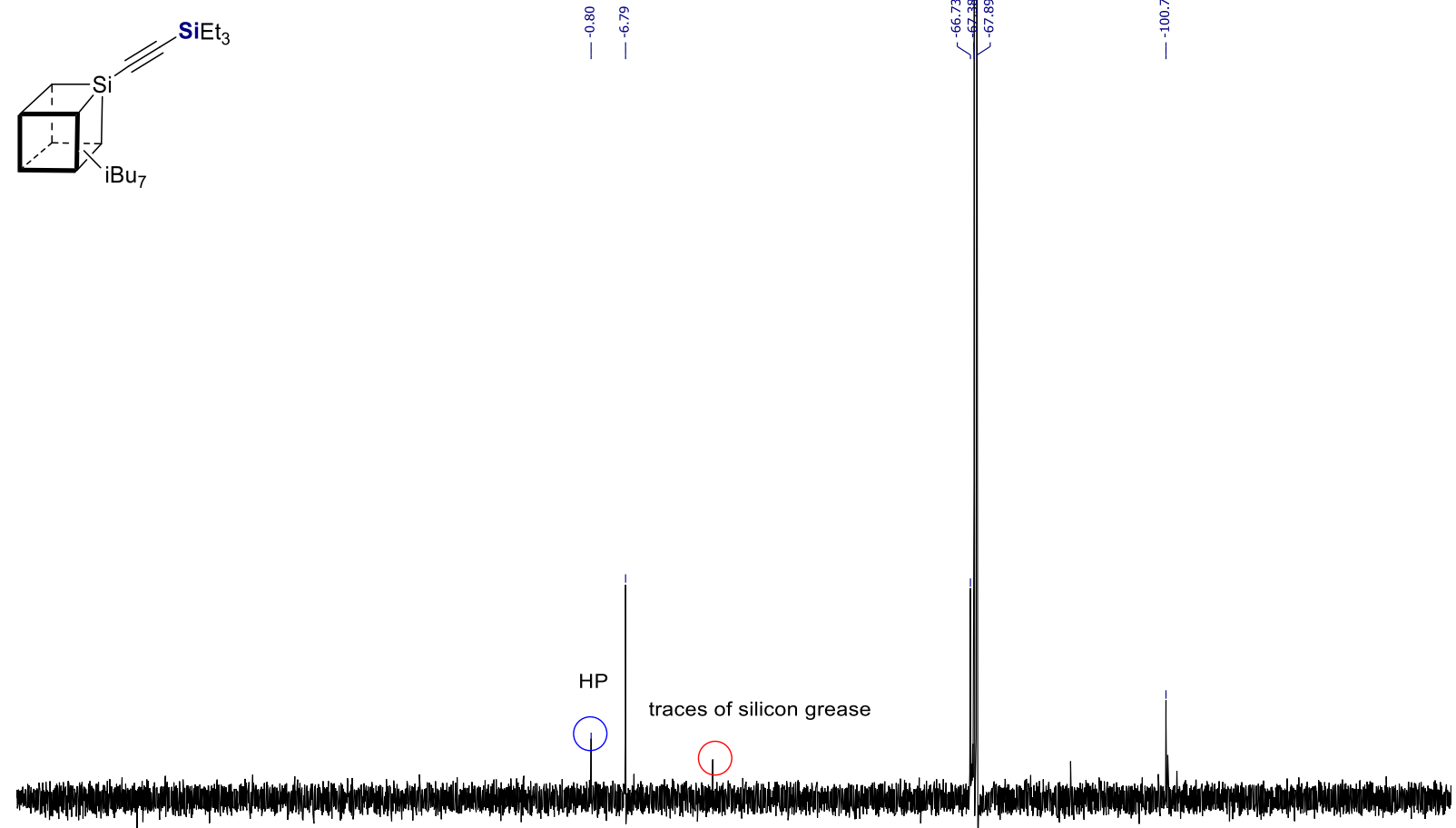

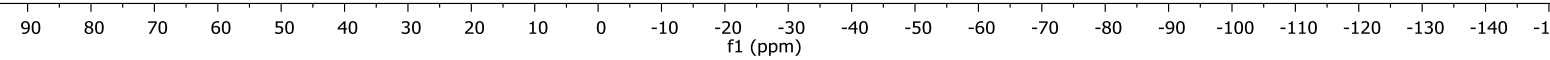

Figure S-53. ${ }^{29} \mathrm{Si} \mathrm{NMR}$ spectrum $\left(79 \mathrm{MHz}, \mathrm{CDCl}_{3}\right)$ of product $\mathrm{P}-5 \mathrm{c}$. 
White solid. Isolated yield $79 \%$.

${ }^{1} \mathrm{H}$ NMR (CDCl, $\left.300 \mathrm{MHz}\right): \delta$ 1.92-1.81 (m, 7H, iBu), 0.96-0.94 (m, 42H, iBu), 0.65-0.58 (m, 14H, iBu), $0.16\left(\mathrm{~s}, 3 \mathrm{H},-\mathrm{SiMe}\left(\mathrm{OSiMe}_{3}\right)_{3}, 0.14\left(\mathrm{~s}, 18 \mathrm{H},-\mathrm{SiMe}\left(\mathrm{OSiMe}_{3}\right)_{3}\right)\right.$.

${ }^{13} \mathrm{C}$ NMR $\left(\mathrm{CDCl}_{3}, 100 \mathrm{MHz}\right): \delta 108.33(\mathrm{C} \equiv \mathrm{C}), 101.63(\mathrm{C} \equiv \mathrm{C}), 25.83,25.65,23.98,22.61,1.75,1.57$.

${ }^{29} \mathrm{Si}$ NMR $\left(\mathrm{CDCl}_{3}, 79 \mathrm{MHz}\right): \delta 10.50,-54.15,-67.29,-67.38,-67.90$.

EA: Anal. calcd for $\mathrm{C}_{37} \mathrm{H}_{84} \mathrm{O}_{14} \mathrm{Si}_{11}$ (\%):C, 41.85, $\mathrm{H}, 7.97$; found: $\mathrm{C}, 42.19 ; \mathrm{H}, 8.23$.
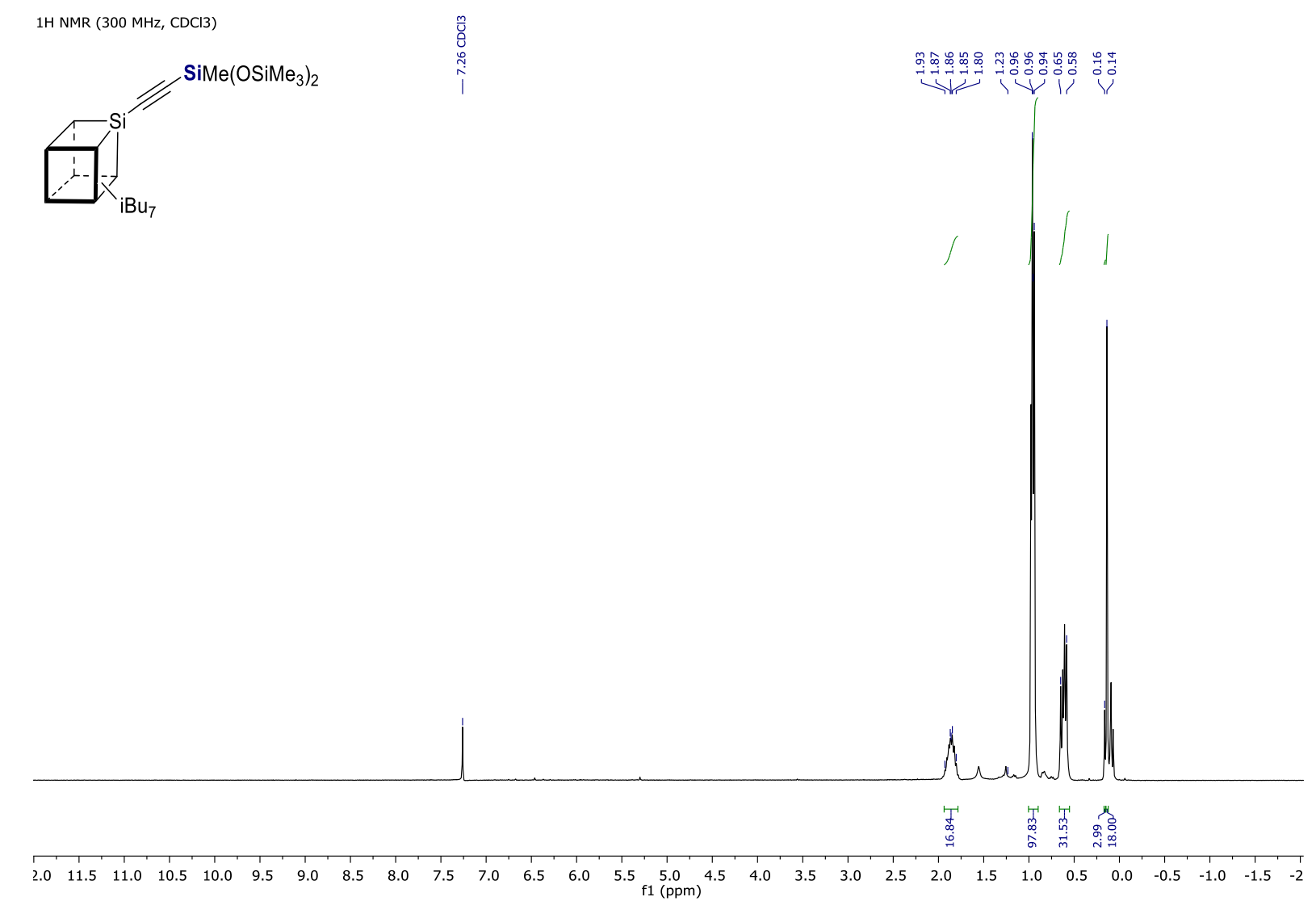

Figure S-54. ${ }^{1} \mathrm{H}$ NMR spectrum $\left(300 \mathrm{MHz}, \mathrm{CDCl}_{3}\right)$ of product $\mathrm{P}-5 \mathrm{~d}$. 

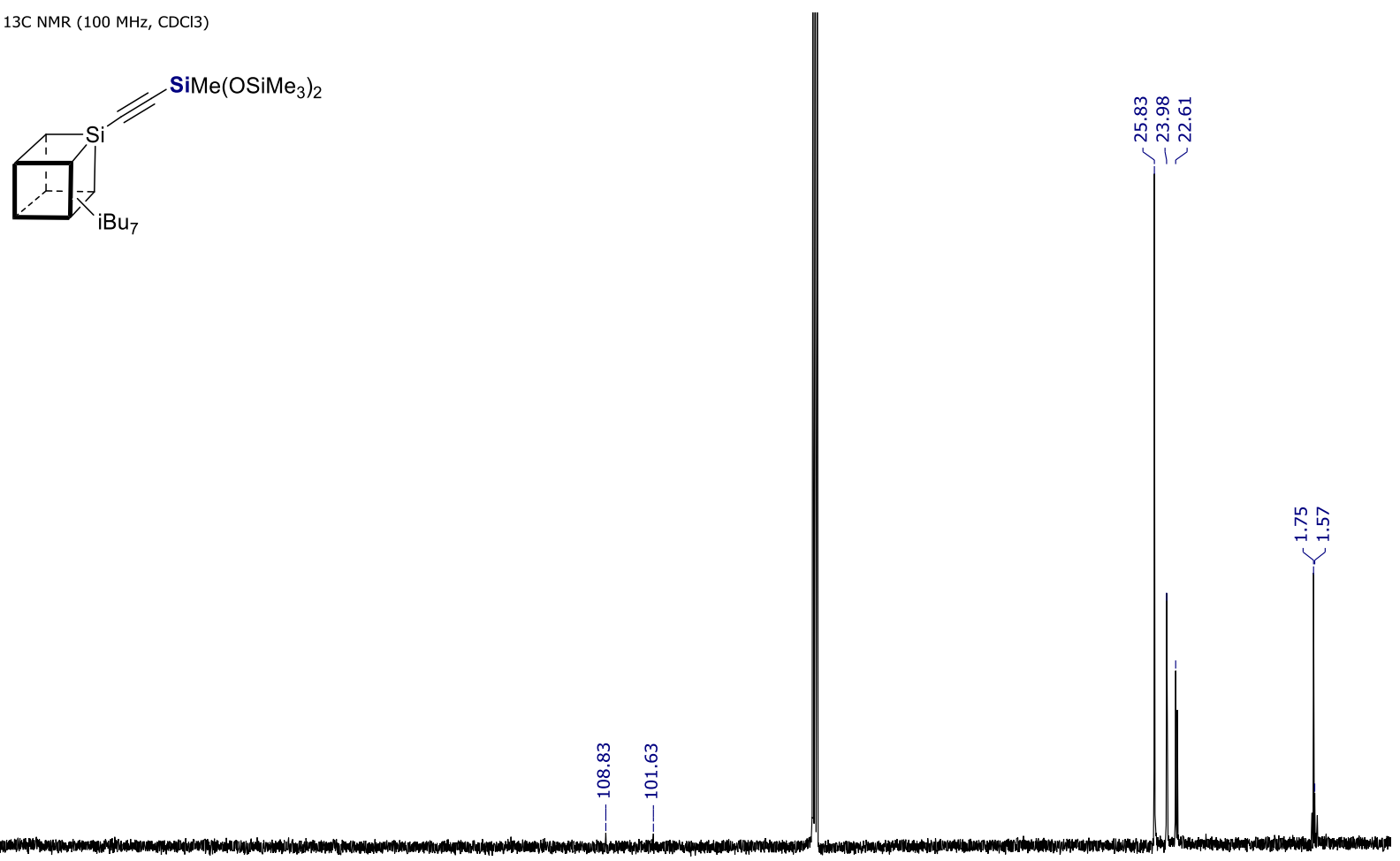

00

Figure S-55. ${ }^{13} \mathrm{C}$ NMR spectrum $\left(100 \mathrm{MHz}, \mathrm{CDCl}_{3}\right)$ of product $\mathrm{P}-5 \mathrm{~d}$.

29Si NMR (79 MHz, CDCl3)
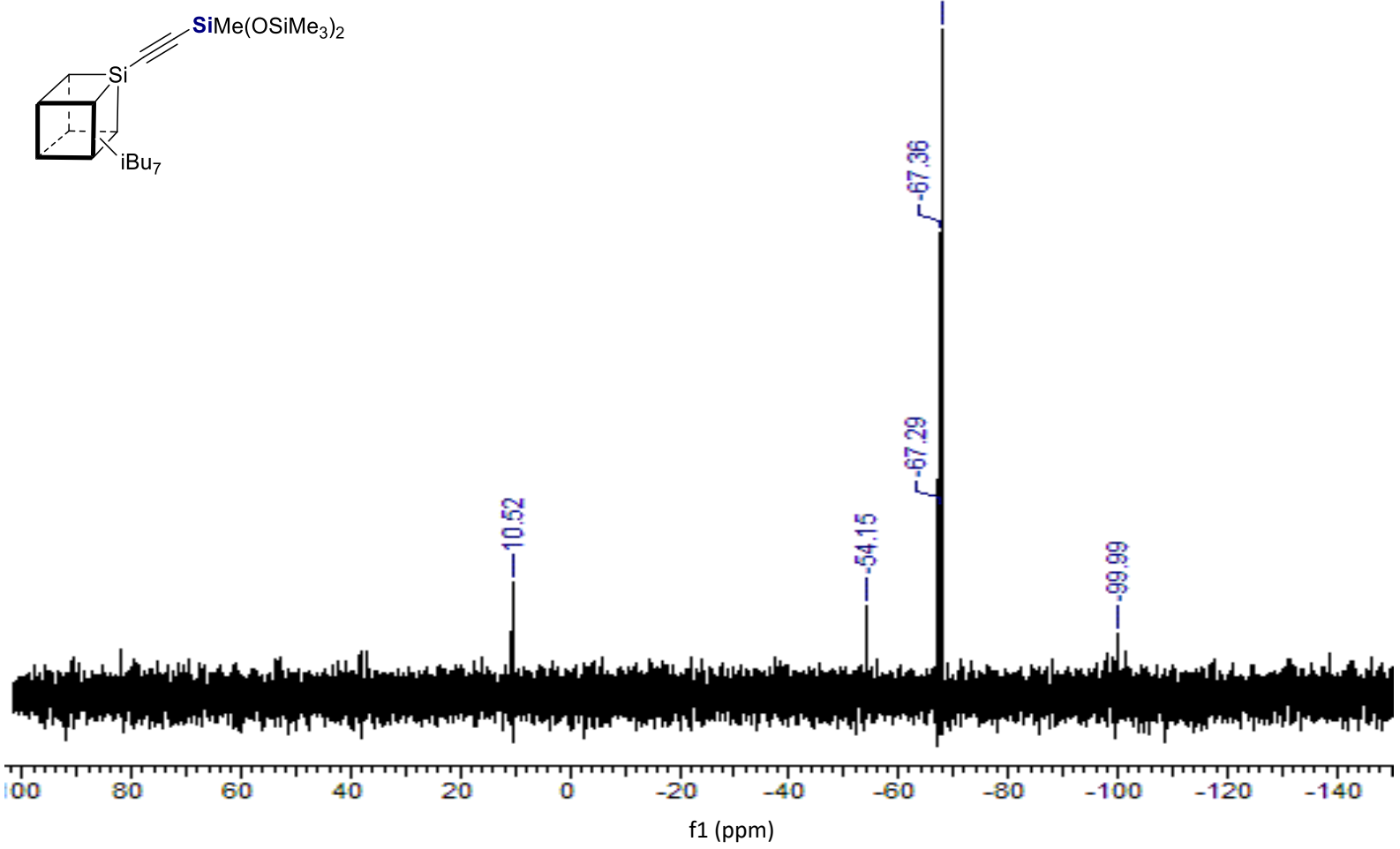

Figure S-56. ${ }^{29}$ Si NMR spectrum $\left(79 \mathrm{MHz}, \mathrm{CDCl}_{3}\right)$ of product $\mathrm{P}-5 \mathrm{~d}$. 


\section{P-6a}

Product obtained as white solid. Isolated yield $85 \%$.

${ }^{1} \mathrm{H}$ NMR $\left(\mathrm{CDCl}_{3}, 400 \mathrm{MHz}\right): \delta$ 7.63-7.19 (m, Ph), $0.36(\mathrm{~s}, 6 \mathrm{H},-\mathrm{SiMe}), 0.18\left(\mathrm{~s}, 12 \mathrm{H},-\mathrm{SiMe}_{2}\right), 0.11(\mathrm{~s}, 18 \mathrm{H}$, $\left.-\mathrm{SiMe}_{3}\right)$.

${ }^{13} \mathrm{C}$ NMR $\left(\mathrm{CDCl}_{3}, 75 \mathrm{MHz}\right): \delta$ 134.21-130.45(Ph), 127.99-127.60, 113.26(C=C), 112.18(C=C), 2.09, -0.14 , -2.74 .

${ }^{29} \mathrm{Si}$ NMR $\left(\mathrm{CDCl}_{3}, 79 \mathrm{MHz}\right): \delta-18.62,-18.64,-63.66,-79.13,-79.47$.

EA: Anal. calcd for $\mathrm{C}_{60} \mathrm{H}_{64} \mathrm{O}_{14} \mathrm{Si}_{12}$ (\%):C, 53.53, $\mathrm{H}, 4.79$; found: $\mathrm{C}, 53.69 ; \mathrm{H}, 4.86$.

$1 \mathrm{H} \mathrm{NMR} \mathrm{(300MHz,} \mathrm{CDCl3)}$

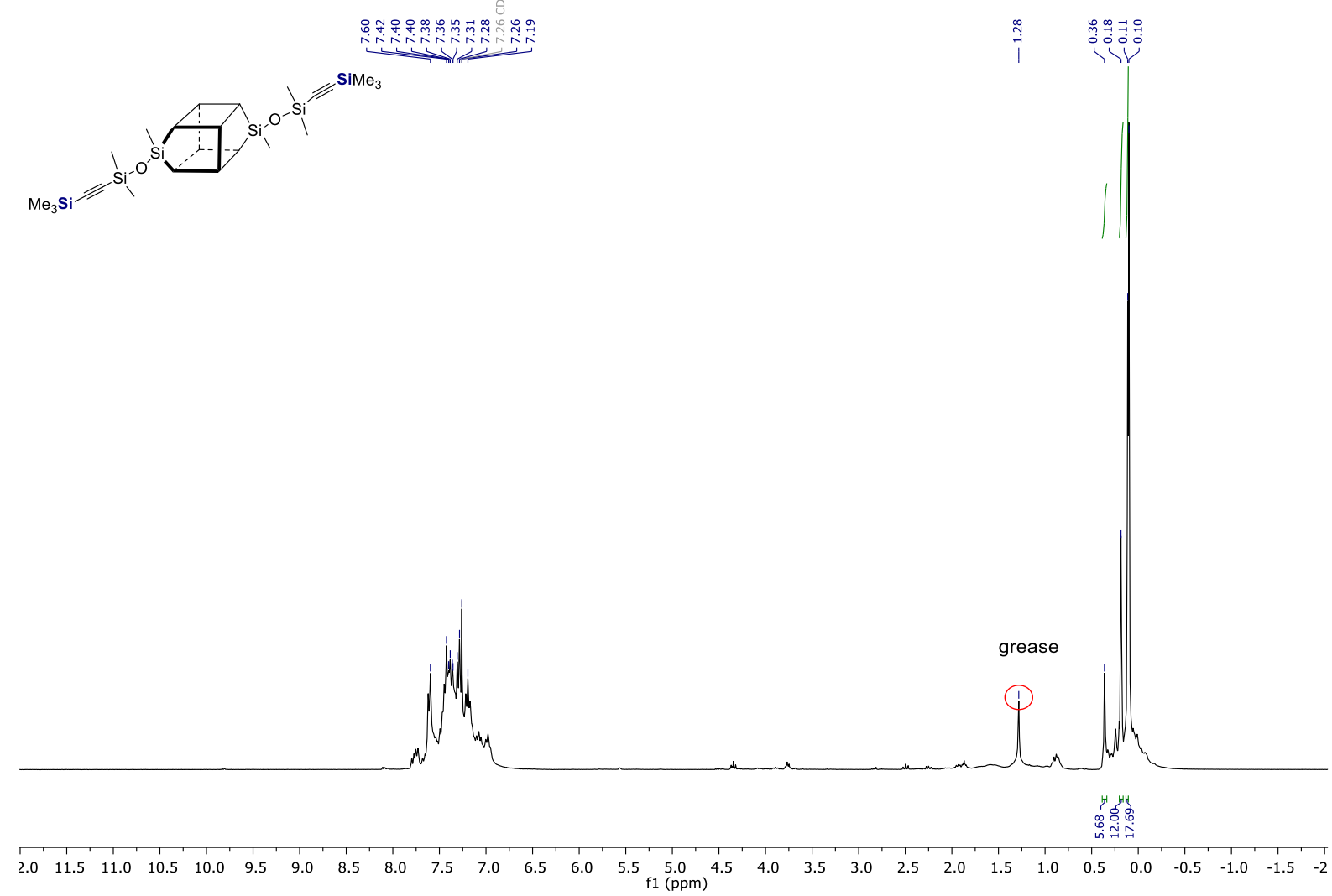

Figure S-57. ${ }^{1} \mathrm{H}$ NMR spectrum $\left(300 \mathrm{MHz}, \mathrm{CDCl}_{3}\right)$ of product $\mathrm{P}-6 \mathrm{a}$. 


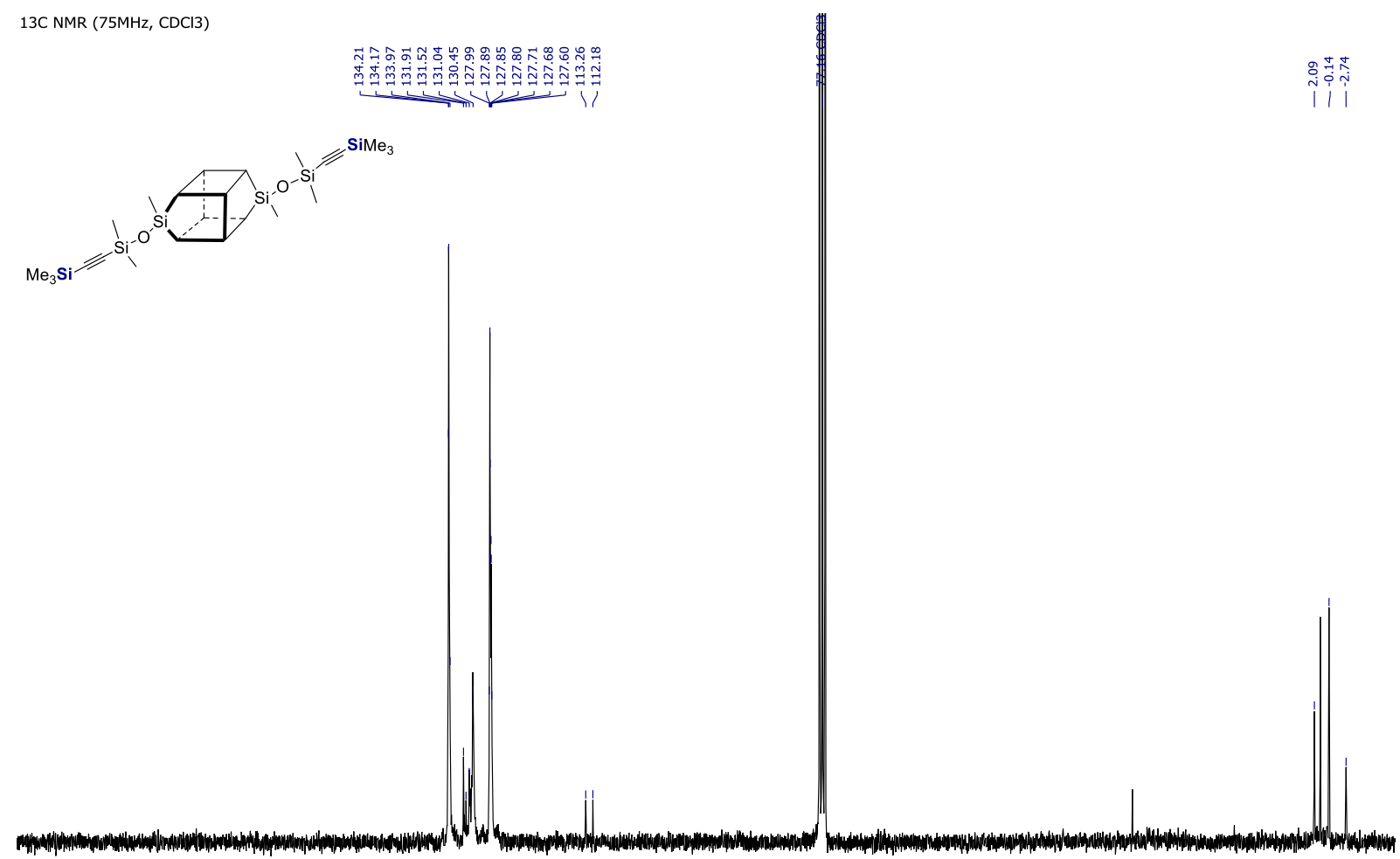

$\begin{array}{lllllllllllllllllllll}1 & 190 & 180 & 170 & 160 & 150 & 140 & 130 & 120 & 110 & \underset{f}{100}(\mathrm{ppm}) & 80 & 70 & 60 & 50 & 40 & 30 & 20 & 10 & 0 & -1\end{array}$

Figure S-58. ${ }^{13} \mathrm{C}$ NMR spectrum $\left(75 \mathrm{MHz}, \mathrm{CDCl}_{3}\right.$ ) of product P-6a.

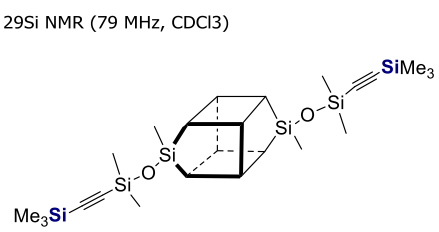

Vं

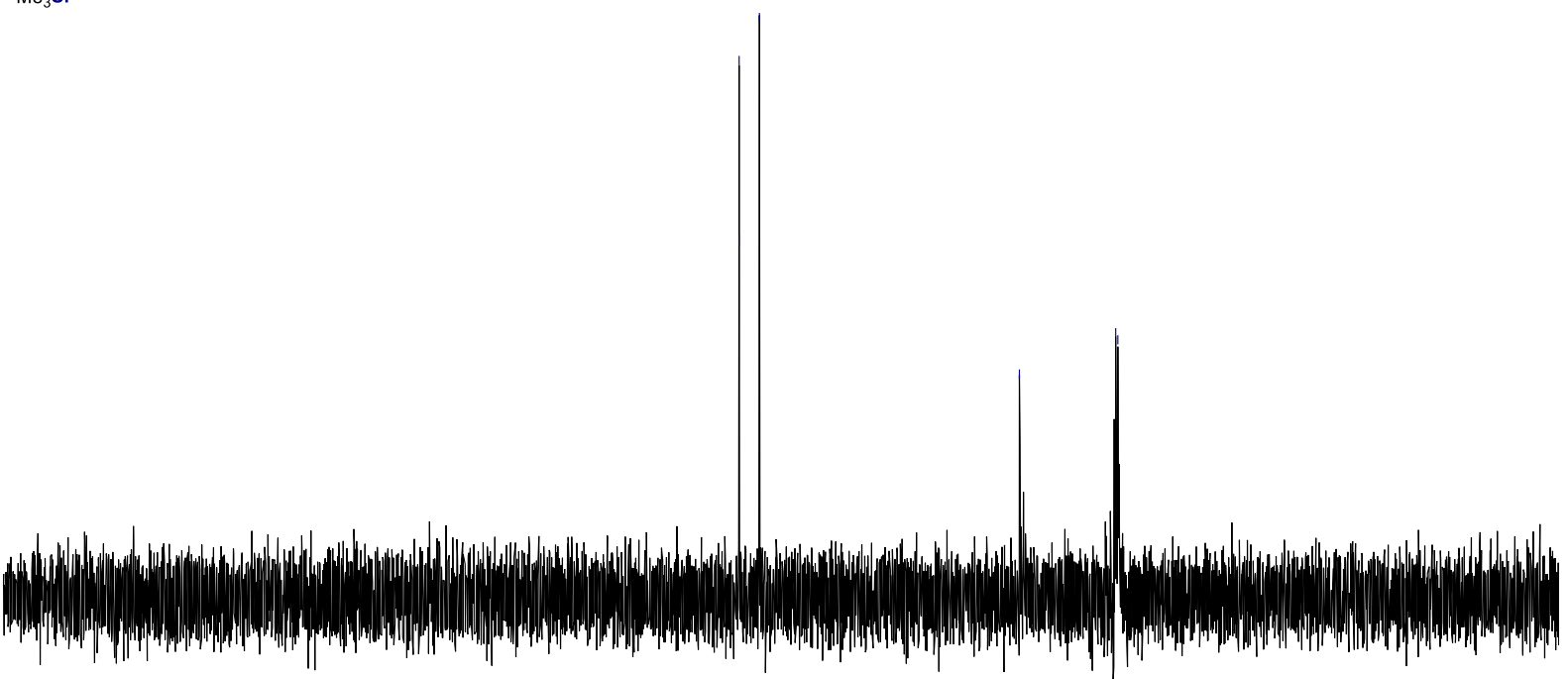

$\begin{array}{llllllllllllllllllllllllll}90 & 80 & 70 & 60 & 50 & 40 & 30 & 20 & 10 & 0 & -10 & \underset{-20}{-20}-30 & -40 & -50 & -60 & -70 & -80 & -90 & -100 & -110 & -120 & -130 & -140 & -1\end{array}$

Figure S-59. ${ }^{29} \mathrm{Si} \mathrm{NMR} \mathrm{spectrum}\left(300 \mathrm{MHz}, \mathrm{CDCl}_{3}\right)$ of product $\mathrm{P}-6 \mathrm{a}$. 


\section{P-6b}

${ }^{1} \mathrm{H}$ NMR $\left(\mathrm{CDCl}_{3}, 300 \mathrm{MHz}\right): \delta$ 7.58-7.15 (m, Ph), $0.34\left(\mathrm{~s}, 12 \mathrm{H},-\mathrm{SiMe}_{2} \mathrm{Ph}\right), 0.20(\mathrm{~s}, 12 \mathrm{H},-\mathrm{Me}), 0.09$ (s, $6 \mathrm{H},-\mathrm{Me})$.

${ }^{13} \mathrm{C}$ NMR $\left(\mathrm{CDCl}_{3}, 75 \mathrm{MHz}\right): \delta 136.61,134.20,134.14,133.81,131.86,130.51,129.52,127.90,114.25$ $(\mathrm{C} \equiv \mathrm{C}), 110.84(\mathrm{C} \equiv \mathrm{C}), 2.08,1.19,-0.90,-2.76$.

${ }^{29} \mathrm{Si}$ NMR $\left(\mathrm{CDCl}_{3}, 79 \mathrm{MHz}\right): \delta-18.37,-18.40,-63.58,-79.04,-79.25,-79.42,-79.60$.

EA: Anal. calcd for $\mathrm{C}_{74} \mathrm{H}_{80} \mathrm{O}_{16} \mathrm{Si}_{14}$ (\%):C, 53.53, $\mathrm{H}, 4.79$; found: $\mathrm{C}, 53.89 ; \mathrm{H}, 5.06$.
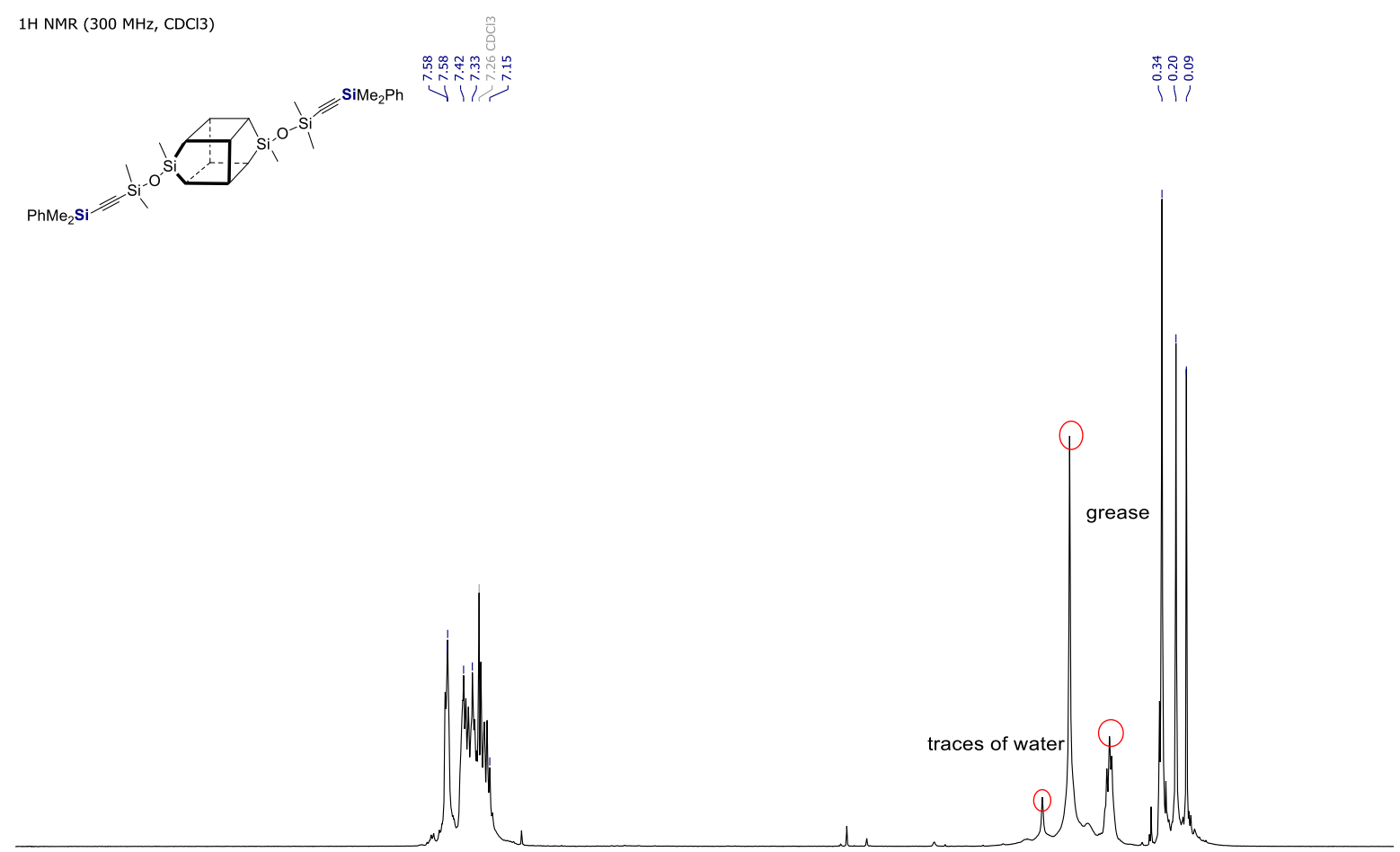

$\begin{array}{lllllllllllllllllllllllllllllllllll}11.5 & 11.0 & 10.5 & 10.0 & 9.5 & 9.0 & 8.5 & 8.0 & 7.5 & 7.0 & 6.5 & 6.0 & 5.5 & 5.0 & 4.5 & 4.0 & 3.5 & 3.0 & 2.5 & 2.0 & 1.5 & 1.0 & 0.5 & 0.0 & -0.5 & -1.0 & -1.5 & -2\end{array}$

Figure S-60. ${ }^{1} \mathrm{H}$ NMR spectrum $\left(300 \mathrm{MHz}, \mathrm{CDCl}_{3}\right.$ ) of product P-6b. 

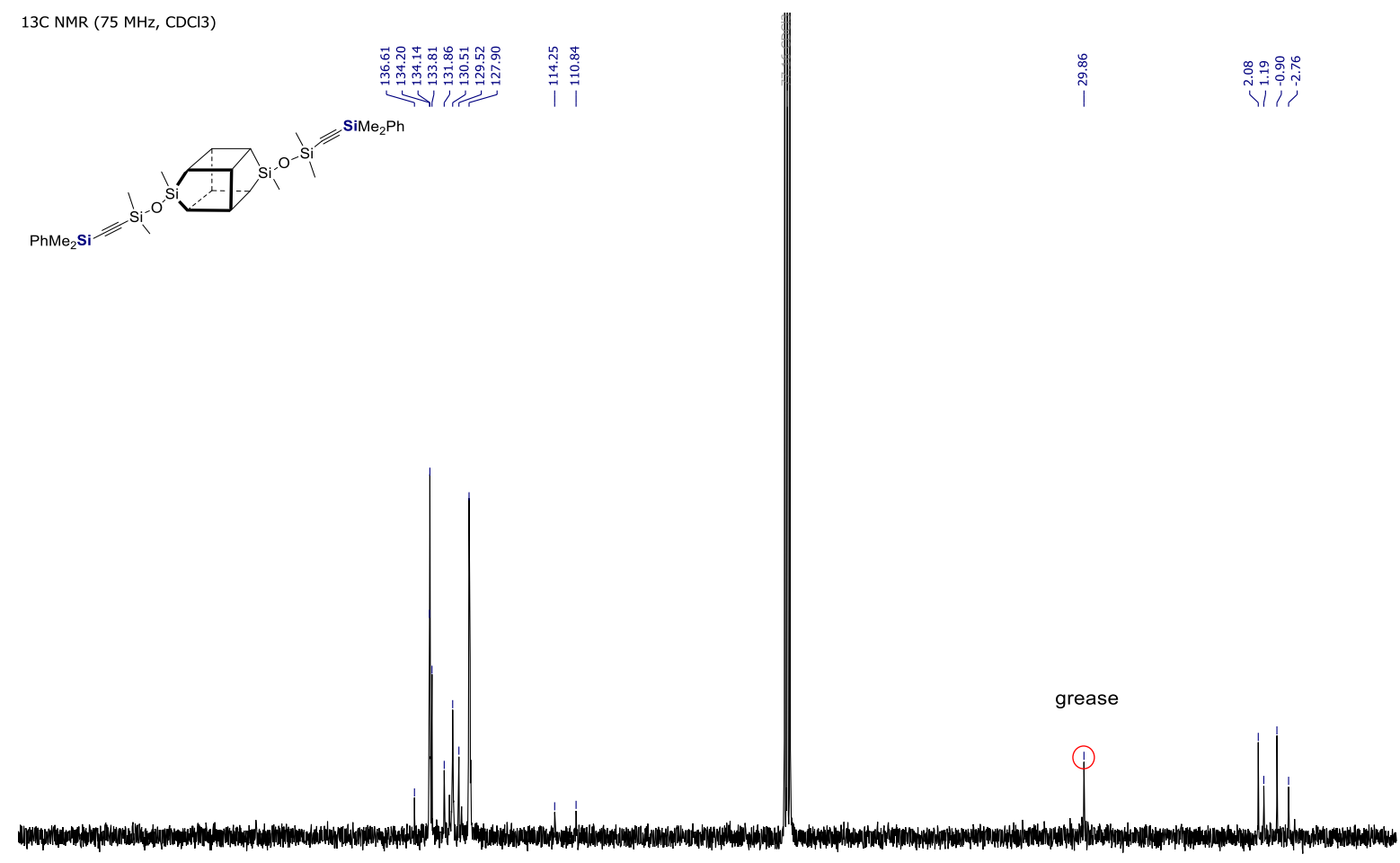

$\begin{array}{lllllllllllllllllllll}190 & 180 & 170 & 160 & 150 & 140 & 130 & 120 & 110 & 100 & \underset{\mathrm{f} 1(\mathrm{ppm})}{90} & 80 & 70 & 60 & 50 & 40 & 30 & 20 & 10 & 0 & -10\end{array}$

Figure S-61. ${ }^{13} \mathrm{C}$ NMR spectrum $\left(75 \mathrm{MHz}, \mathrm{CDCl}_{3}\right)$ of product P-6b.

29Si NMR(79MHz, CDCl3)

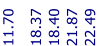

i í

至

PhMe $\mathrm{Si}_{1}=\mathrm{Si}^{\mathrm{O}}$

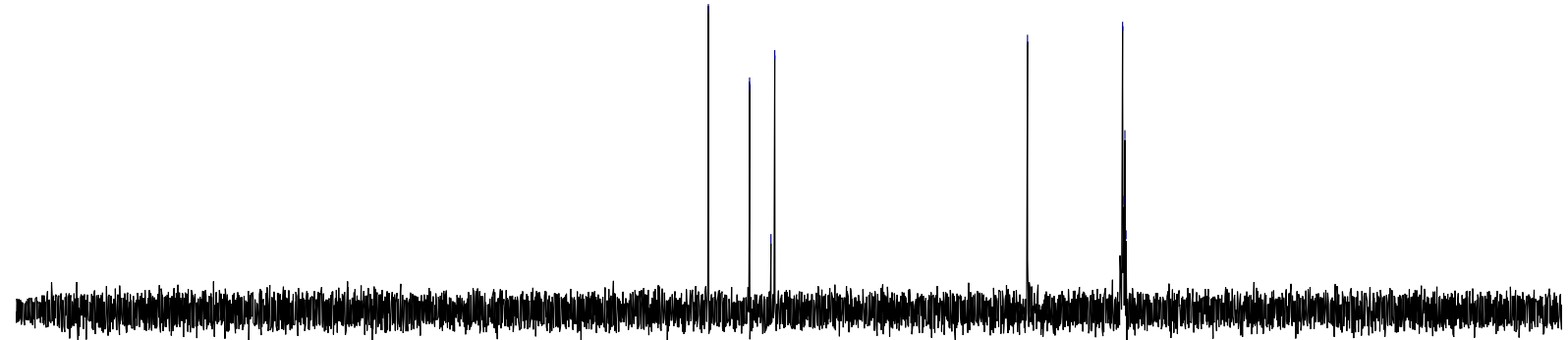

\begin{tabular}{rlllllllllllllllllllllllllllllllllll}
\hline 00 & 90 & 80 & 70 & 60 & 50 & 40 & 30 & 20 & 10 & 0 & -10 & -20 & -30 & -40 & -50 & -60 & -70 & -80 & -90 & -100 & -110 & -120 & -130 & -140 & -1
\end{tabular}

Figure S-62. ${ }^{29} \mathrm{Si} \mathrm{NMR}$ spectrum $\left(79 \mathrm{MHz}, \mathrm{CDCl}_{3}\right)$ of product P-6b. 


\section{P-6c}

White solid. Isolated yield $75 \%$,

Product accompanied by homocoupling product of S-c, i.e. 1,2-bis(triethylsilyl)ethene (HP).

${ }^{1} \mathrm{H} \mathrm{NMR}\left(\mathrm{CDCl}_{3}, 500 \mathrm{MHz}\right): \delta$ 7.63-7.21 (m, 40H, Ph), 0.98- 0.85 (t, 18H, Et), 0.49-0.57 (m, 12H, Et), $0.16(\mathrm{~s}, 12 \mathrm{H}, \mathrm{Me})$.

${ }^{13} \mathrm{C} \mathrm{NMR}\left(\mathrm{CDCl}_{3}, 500 \mathrm{MHz}\right): \delta 134.07(\mathrm{Ph}), 130.36,127.75-127.60,113.57$ (C=C), 110.69 (C=C), 7.38, 4.16, 2.03, 1.04, -2.90.

${ }^{29} \mathrm{Si}$ NMR $\left(\mathrm{CDCl}_{3}, 99 \mathrm{MHz}\right): \delta-7.94,-18.82,-66.97,-78.97,-79.13$

EA: Anal. calcd for $\mathrm{C}_{70} \mathrm{H}_{88} \mathrm{O}_{16} \mathrm{Si}_{14}$ (\%):C, 53.26, $\mathrm{H}, 5.62$; found: $\mathrm{C}, 53.49 ; \mathrm{H}, 5.76$.
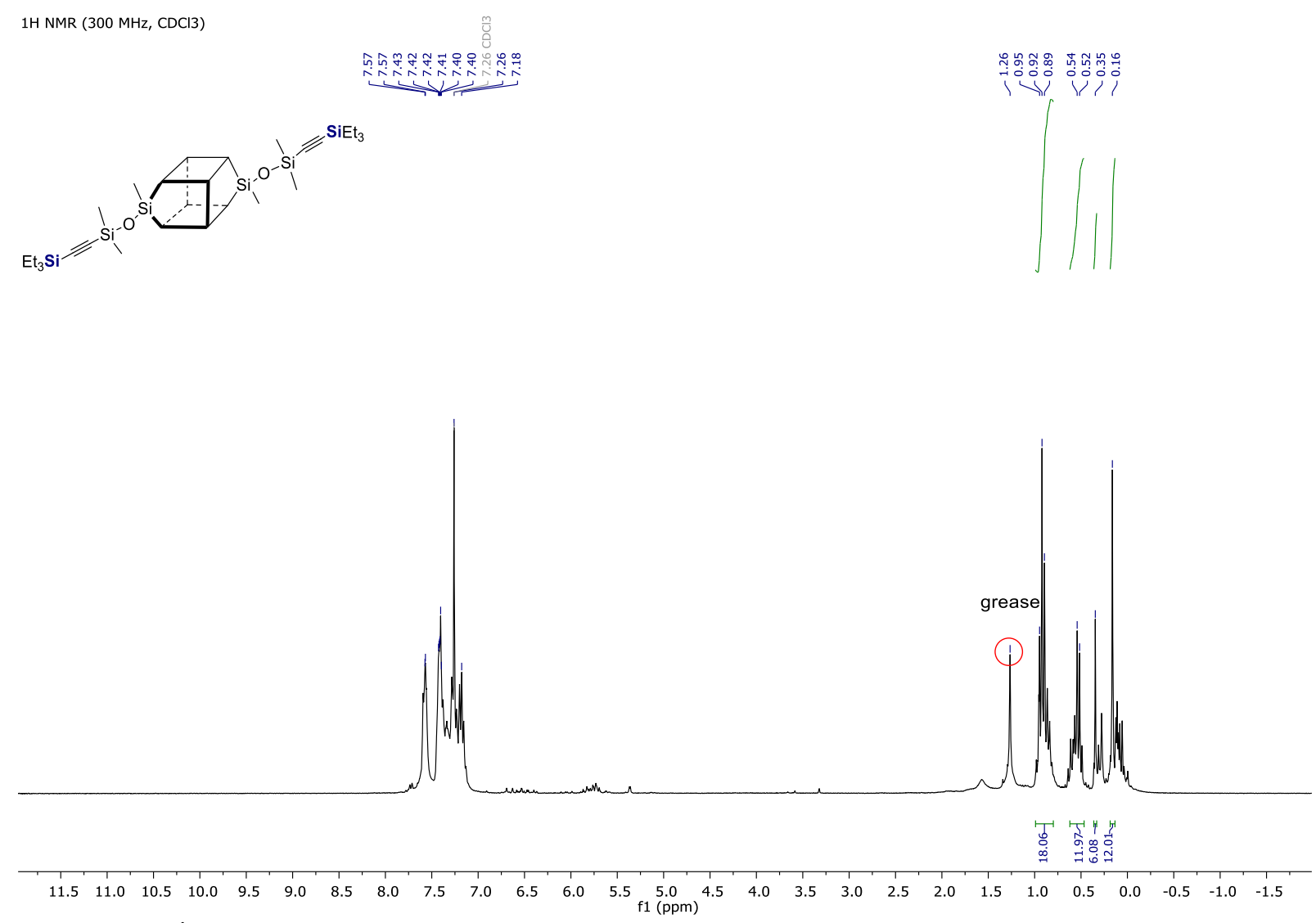

Figure S-63. ${ }^{1} \mathrm{H}$ NMR spectrum $\left(300 \mathrm{MHz}, \mathrm{CDCl}_{3}\right)$ of product $\mathrm{P}-6 \mathrm{c}$. 


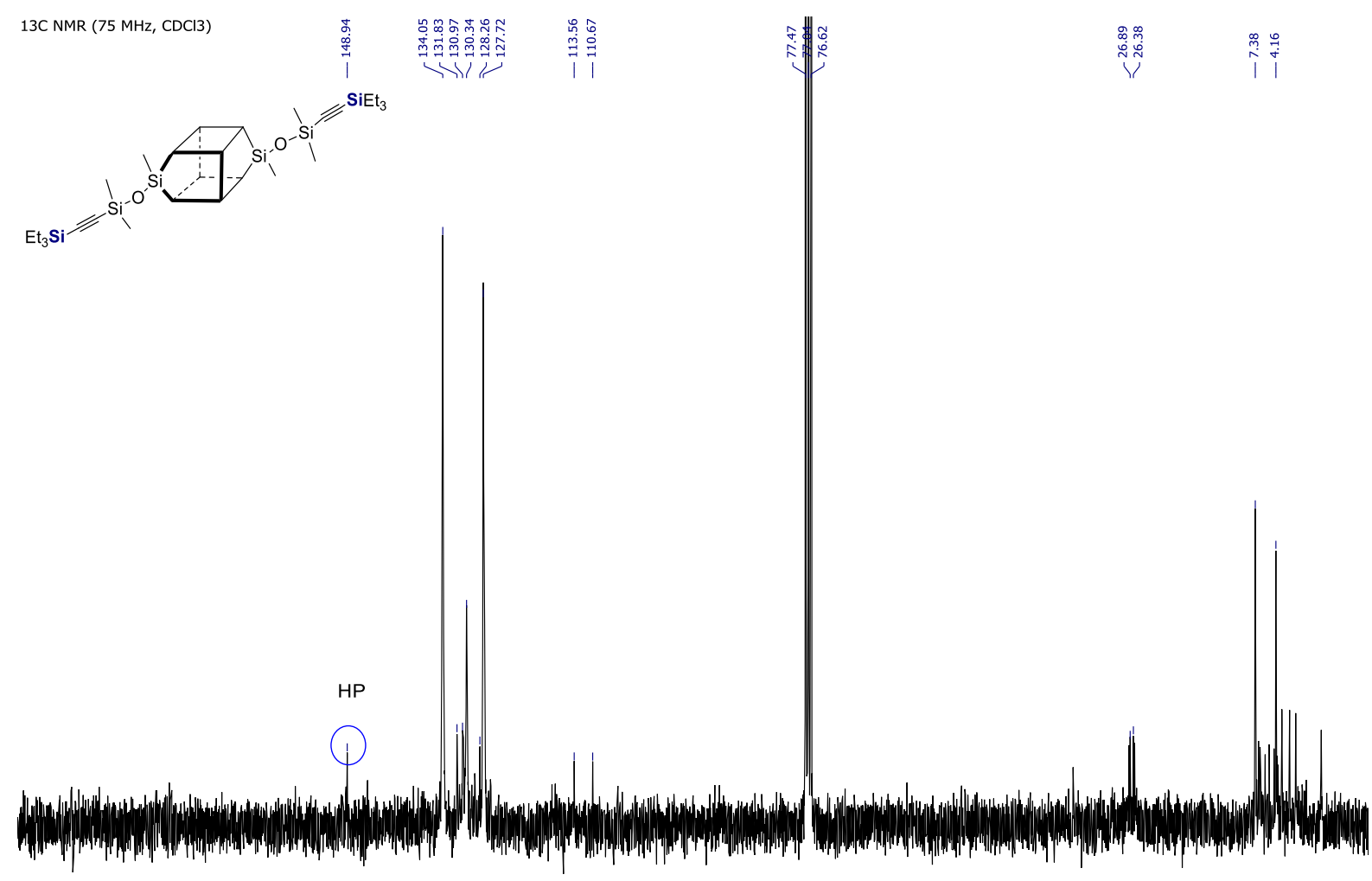

$\begin{array}{llllllllllllllllllllllll}0 & 190 & 180 & 170 & 160 & 150 & 140 & 130 & 120 & 110 & \underset{\mathrm{f} 1(\mathrm{ppm})}{100} & 80 & 70 & 60 & 50 & 40 & 30 & 20 & 10 & 0 & -1\end{array}$

Figure S-64. ${ }^{13} \mathrm{C}$ NMR spectrum $\left(75 \mathrm{MHz}, \mathrm{CDCl}_{3}\right.$ ) of product P-6c.

29Si NMR $(79 \mathrm{MHz}, \mathrm{CDCl} 3)$

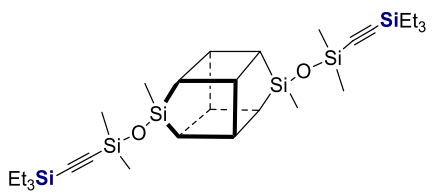

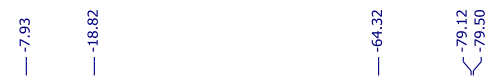

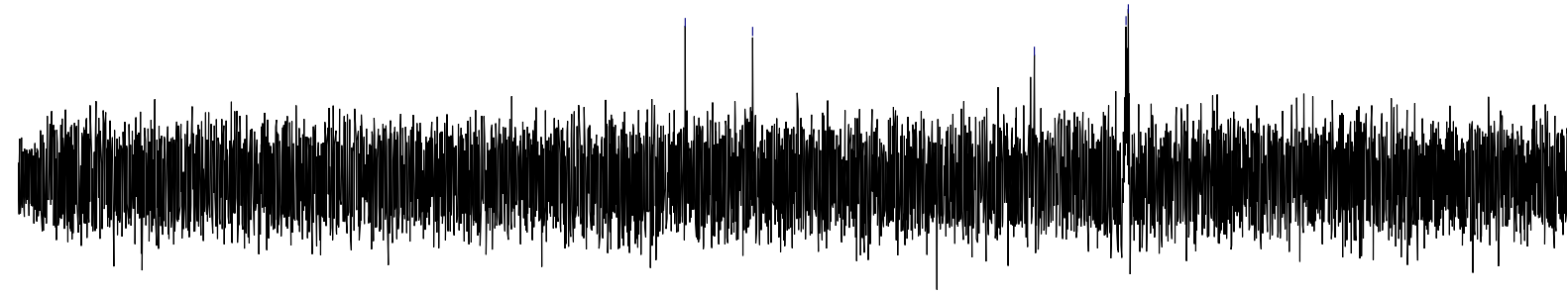

$\begin{array}{lllllllllllllllllllllllll}90 & 80 & 70 & 60 & 50 & 40 & 30 & 20 & 10 & 0 & -10 & -20 & -30 & -40 & -50 & -60 & -70 & -80 & -90 & -100 & -110 & -120 & -130 & -140 & -1\end{array}$

Figure S-65. ${ }^{29} \mathrm{Si} \mathrm{NMR} \mathrm{spectrum}\left(79 \mathrm{MHz}, \mathrm{CDCl}_{3}\right)$ of product P-6c. 


\section{P-7a}

Crude NMR analyses - performed for the post-reaction mixture.

Product accompanied by homocoupling product of S-a, i.e. 1,2-bis(dimethylphenylsilyl)ethene (HP).

${ }^{1} \mathrm{H}$ NMR $\left(\mathrm{CDCl}_{3}, 300 \mathrm{MHz}\right): \delta$ 7.48-6.96 (m, Ph), 0.10-0.00 (overlapped, -SiMe 3 ).

${ }^{13} \mathrm{C}$ NMR $\left(\mathrm{CDCl}_{3}, 75 \mathrm{MHz}\right): \delta 134.43,134.04,130.22,129.18-128.37,127.60,115.23(\mathrm{C} \equiv \mathrm{C})$, 113.21(C $\equiv C),-0.23,-2.29$.

${ }^{29} \mathrm{Si} \mathrm{NMR}\left(\mathrm{CDCl}_{3}, 99 \mathrm{MHz}\right): \delta-1.54(\mathrm{HP}),-17.39,-17.46,-21,90$ (silicon grease), -74.94, -78.64.
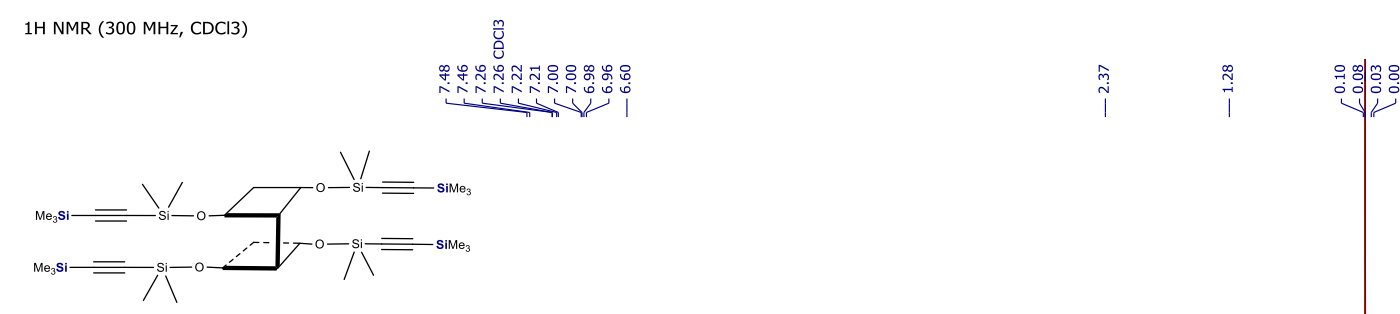

$\begin{array}{lllllllllllllllllllllllllllll}11.5 & 11.0 & 10.5 & 10.0 & 9.5 & 9.0 & 8.5 & 8.0 & 7.5 & 7.0 & 6.5 & 6.0 & 5.5 & \begin{array}{c}5.0 \\ (\mathrm{ppm})\end{array} & 4.5 & 4.0 & 3.5 & 3.0 & 2.5 & 2.0 & 1.5 & 1.0 & 0.5 & 0.0 & -0.5 & -1.0 & -1.5\end{array}$

Figure S-66. ${ }^{1} \mathrm{H}$ NMR spectrum $\left(300 \mathrm{MHz}, \mathrm{CDCl}_{3}\right)$ of product $\mathrm{P}-7 \mathrm{a}$. 


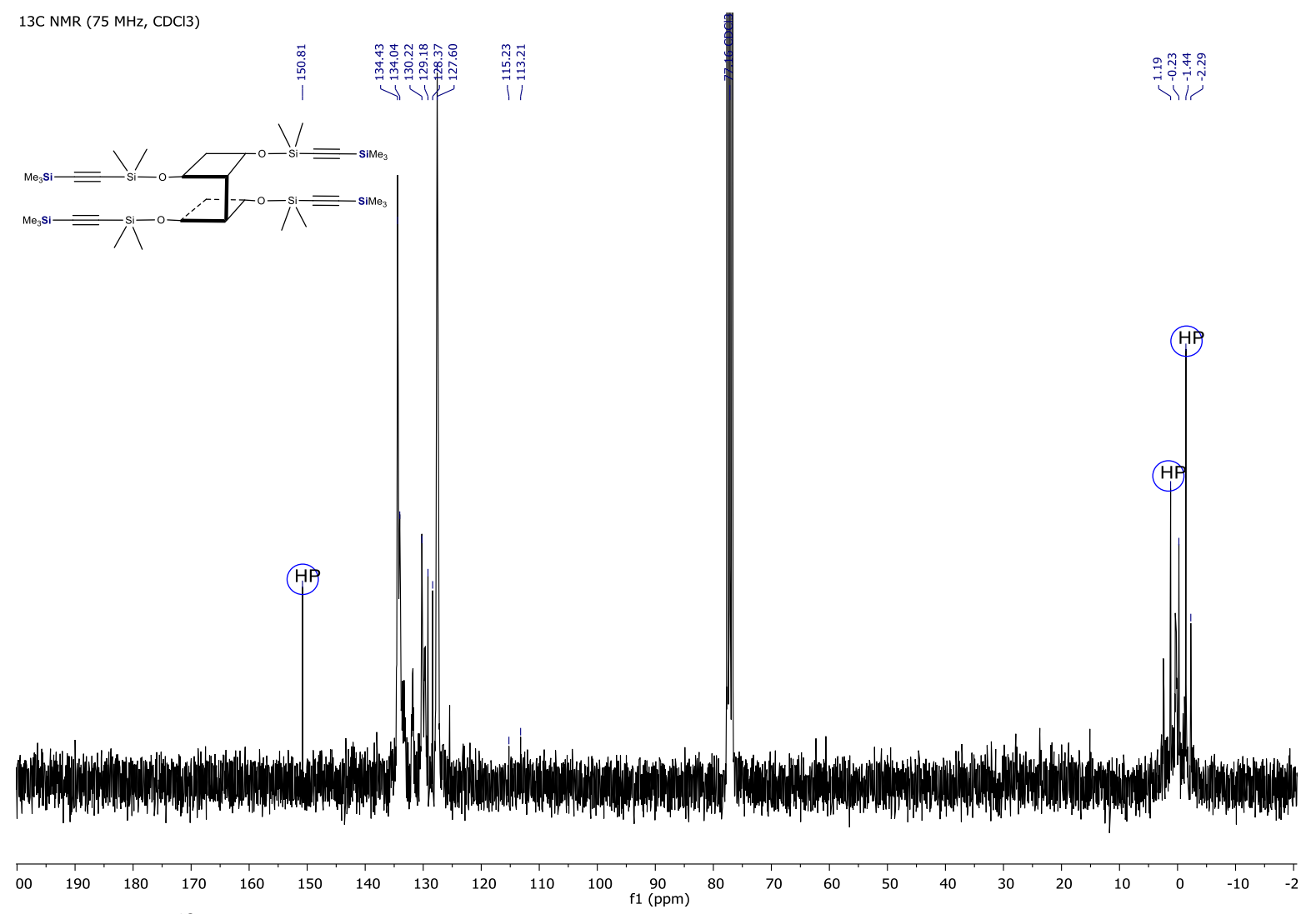

Figure S-67. ${ }^{13} \mathrm{C}$ NMR spectrum $\left(75 \mathrm{MHz}, \mathrm{CDCl}_{3}\right.$ ) of product P-7a. 
${ }^{29} \mathrm{Si} \mathrm{NMR} \mathrm{(not} \mathrm{isolated} \mathrm{sample} \mathrm{of} \mathrm{P-7a):}$
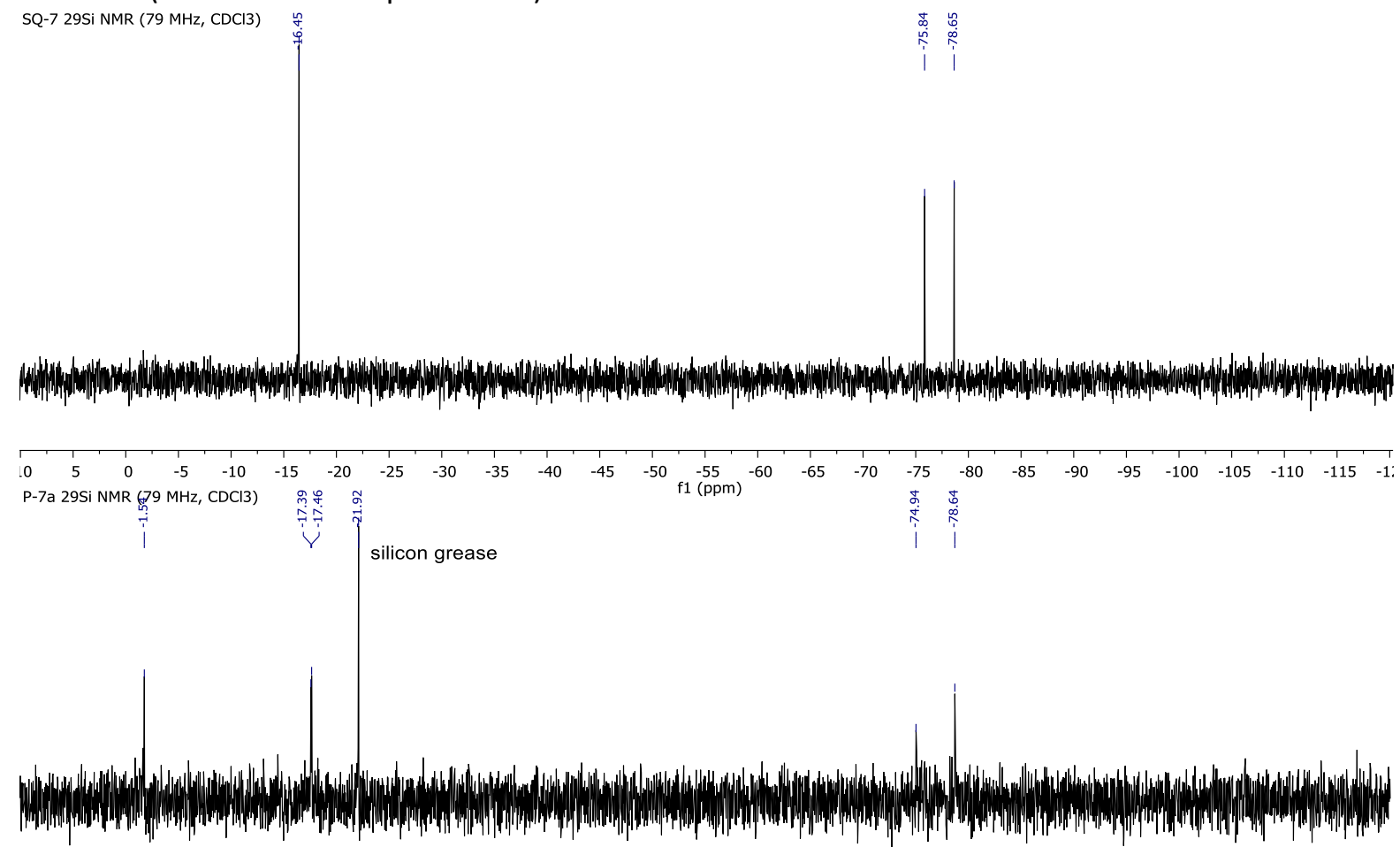

\begin{tabular}{llllllllllllllllllllllllllllllllll}
\hline 10 & 5 & 0 & -5 & -10 & -15 & -20 & -25 & -30 & -35 & -40 & -45 & -50 & -55 & -60 & -65 & -70 & -75 & -80 & -85 & -90 & -95 & -100 & -105 & -110 & -115 & -1
\end{tabular}

Figure S-68. ${ }^{295 i} \mathrm{NMR}$ spectrum $\left(79 \mathrm{MHz}, \mathrm{CDCl}_{3}\right)$ of SQ-7 and product P-7a. 
Crude NMR analyses - performed for the post-reaction mixture.

Product accompanied by homocoupling product of S-c, i.e. 1,2-bis(triethylsilyl)ethene (HP).

${ }^{1} \mathbf{H}$ NMR $\left(\mathrm{CDCl}_{3}, 300 \mathrm{MHz}\right): \delta$ 7.53-7.16 (m, Ph), 1.05-0.30 (overlapped, $\left.-\mathrm{SiEt}_{3}\right) .{ }^{13} \mathrm{C}$ NMR $\left(\mathrm{CDCl}_{3}, 75\right.$ $\mathrm{MHz}): \delta 149.06(\mathrm{HP}), 134.64,134.51,132.81,131.60,130.17,127.51,113.89(\mathrm{C} \equiv \mathrm{C}), 110.60(\mathrm{C} \equiv \mathrm{C}), 7.45$, 4.18, 2.57 .

${ }^{29} \mathrm{Si}$ NMR $\left(\mathrm{CDCl}_{3}, 99 \mathrm{MHz}\right): \delta-1.67(\mathrm{HP}),-8.33,-17.99,-75.87,-78.68$.
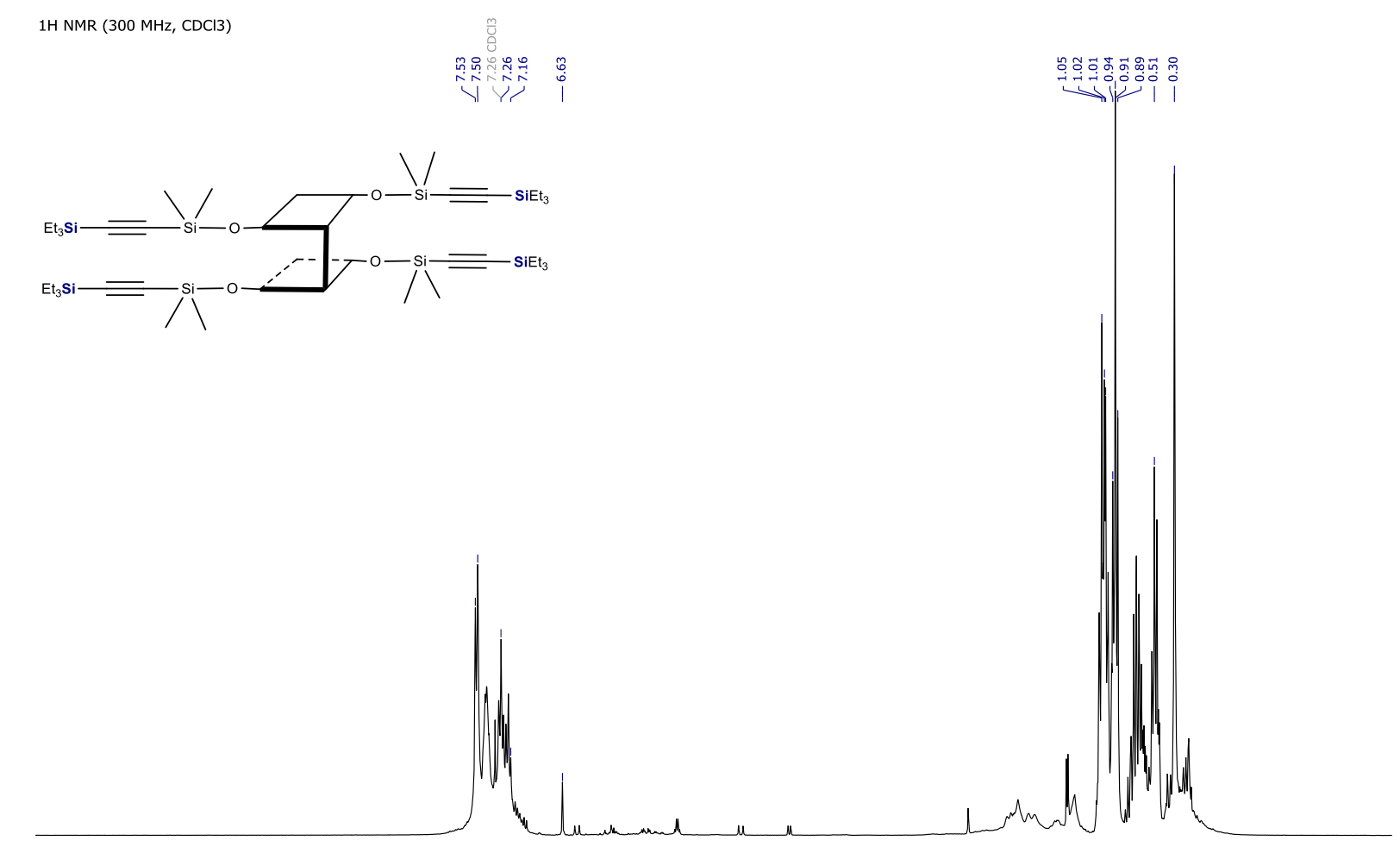

\begin{tabular}{lllllllllllllllllllllllllllllllllllll}
\hline 2.0 & 11.5 & 11.0 & 10.5 & 10.0 & 9.5 & 9.0 & 8.5 & 8.0 & 7.5 & 7.0 & 6.5 & 6.0 & 5.5 & 5.0 & 4.5 & 4.0 & 3.5 & 3.0 & 2.5 & 2.0 & 1.5 & 1.0 & 0.5 & 0.0 & -0.5 & -1.0 & -1.5
\end{tabular}

Figure S-69. ${ }^{1} \mathrm{H}$ NMR spectrum $\left(300 \mathrm{MHz}, \mathrm{CDCl}_{3}\right)$ of product $\mathrm{P}-7 \mathrm{c}$. 


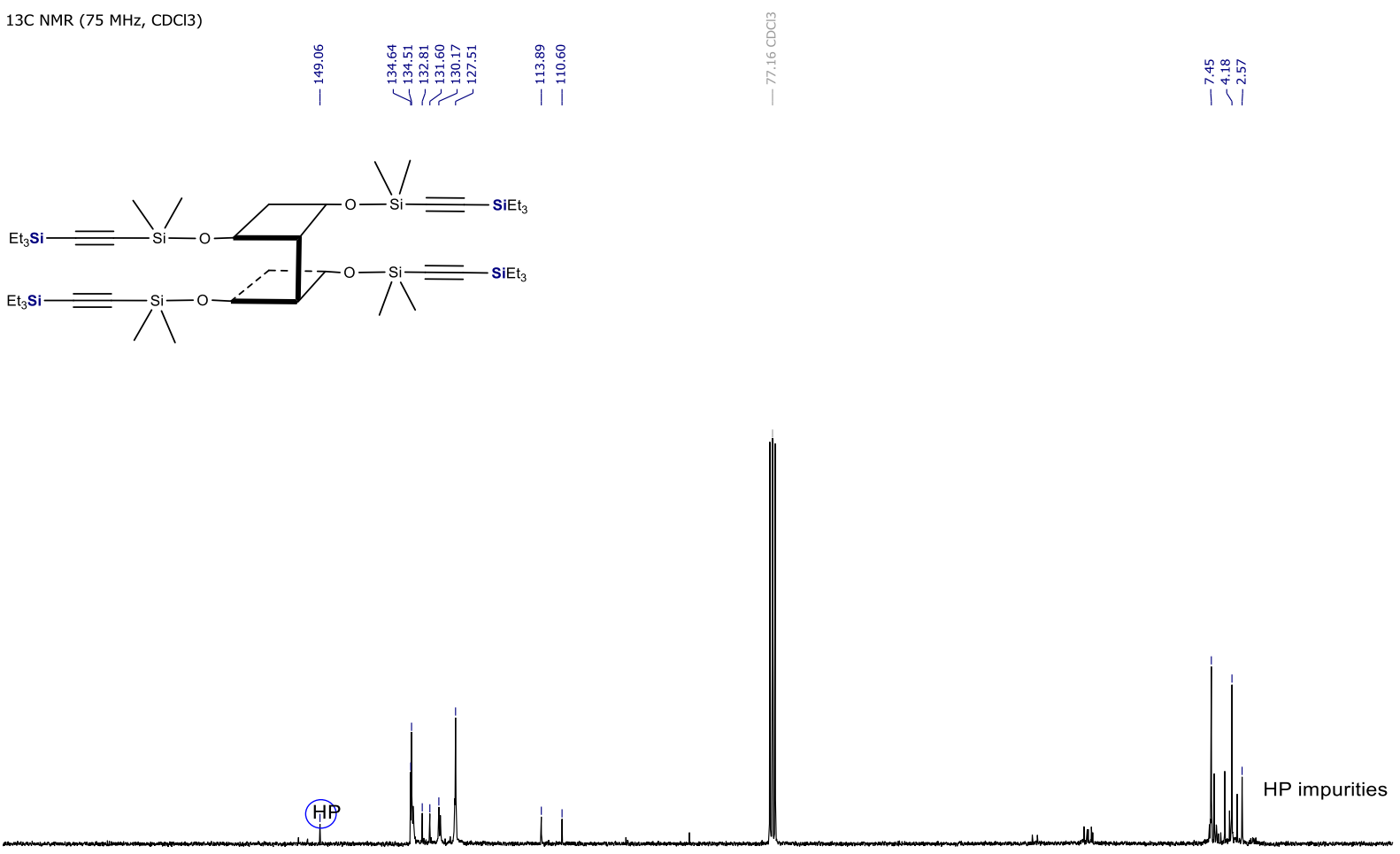

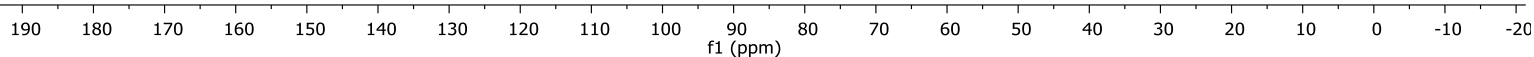

Figure S-70. ${ }^{13} \mathrm{C}$ NMR spectrum $\left(75 \mathrm{MHz}, \mathrm{CDCl}_{3}\right)$ of product P-7c. 

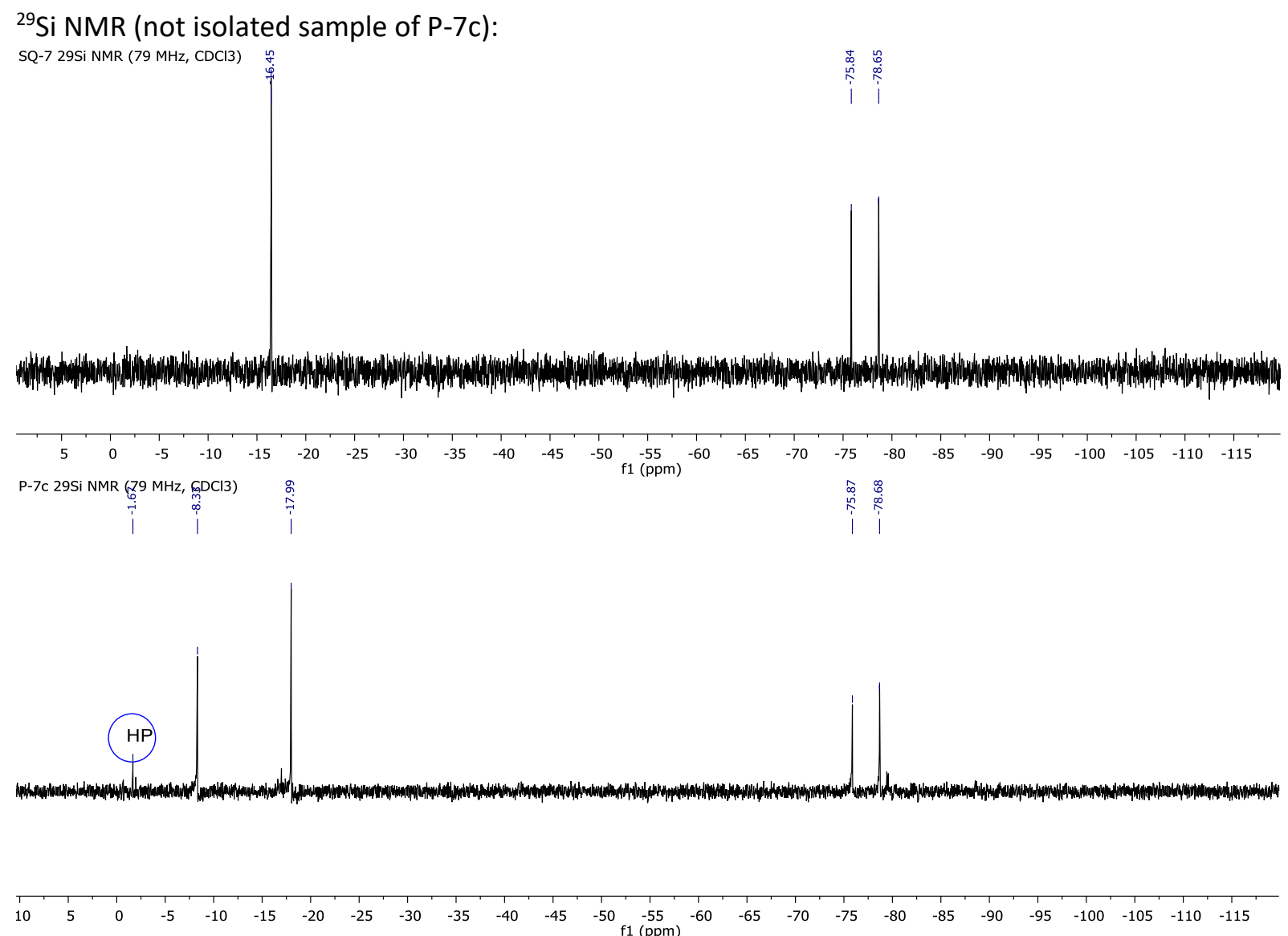

Figure S-71. ${ }^{29} \mathrm{Si} \mathrm{NMR} \mathrm{spectrum} \mathrm{(} 79 \mathrm{MHz}, \mathrm{CDCl}_{3}$ ) of SQ-7 and product $\mathrm{P}-7 \mathrm{c}$. 